San Jose State University

SJSU ScholarWorks

Master's Theses

Master's Theses and Graduate Research

Fall 2015

\title{
Utilization of Differential Thrust for Lateral/Directional Stability of a Commercial Aircraft with a Damaged Vertical Stabilizer
}

Long Lu

San Jose State University

Follow this and additional works at: https://scholarworks.sjsu.edu/etd_theses

\section{Recommended Citation}

Lu, Long, "Utilization of Differential Thrust for Lateral/Directional Stability of a Commercial Aircraft with a Damaged Vertical Stabilizer" (2015). Master's Theses. 4649.

DOI: https://doi.org/10.31979/etd.4ys8-zqt7

https://scholarworks.sjsu.edu/etd_theses/4649

This Thesis is brought to you for free and open access by the Master's Theses and Graduate Research at SJSU ScholarWorks. It has been accepted for inclusion in Master's Theses by an authorized administrator of SJSU ScholarWorks. For more information, please contact scholarworks@sjsu.edu. 


\title{
UTILIZATION OF DIFFERENTIAL THRUST FOR LATERAL/DIRECTIONAL STABILITY OF A COMMERCIAL AIRCRAFT WITH A DAMAGED VERTICAL STABILIZER
}

\author{
A Thesis \\ Presented to \\ The Faculty of the Department of Aerospace Engineering \\ San José State University
}

In Partial Fulfillment

of the Requirements for the Degree

Master of Science

by

Long K. Lu

December 2015 
(C) 2015

Long K. Lu

ALL RIGHTS RESERVED 
The Designated Thesis Committee Approves the Thesis Titled

UTILIZATION OF DIFFERENTIAL THRUST FOR LATERAL/DIRECTIONAL STABILITY OF A COMMERCIAL AIRCRAFT WITH A DAMAGED VERTICAL STABILIZER

\author{
by \\ Long K. Lu \\ APPROVED FOR THE DEPARTMENT OF AEROSPACE ENGINEERING \\ SAN JOSÉ STATE UNIVERSITY
}

December 2015

Dr. Kamran Turkoglu

Department of Aerospace Engineering

Dr. Nikos Mourtos

Department of Aerospace Engineering

Dr. Ping Hsu

Department of Electrical Engineering 


\section{ABSTRACT \\ UTILIZATION OF DIFFERENTIAL THRUST FOR LATERAL/DIRECTIONAL STABILITY OF A COMMERCIAL AIRCRAFT WITH A DAMAGED VERTICAL STABILIZER}

by Long K. Lu

This thesis investigates the utilization of differential thrust to help a commercial aircraft with a damaged vertical stabilizer regain its lateral/directional stability. In the event of an aircraft losing its vertical stabilizer, the consequential loss of the lateral/directional stability is likely to cause a fatal crash. In this thesis, the damaged aircraft model is constructed, and the lateral/directional dynamic stability and frequency domain analyses are conducted. The propulsion dynamics of the aircraft are modeled as a system of differential equations with engine time constant and time delay terms to study the engine response time with respect to a differential thrust input. The novel differential thrust control module is presented to map the rudder input to differential thrust input. Then, the differential thrust based control strategies such as linear quadratic regulator (LQR), model reference adaptive system (MRAS), and $H_{\infty}$ loop-shaping based robust control system are proposed to be utilized to help maintain stability and control of the damaged aircraft. For each type of control system design, robustness and sensitivity analysis is also conducted to test the performance of each control system in the presence of noise and uncertainty. Results demonstrate successful applications of such control methodologies as the damaged aircraft can achieve stability under feasible control efforts and without any actuator saturation. Finally, a comparison study of three control systems is conducted to investigate the merits and limits of each control system. Overall, the $H_{\infty}$ loop-shaping based robust control system was found to have the most remarkable results for stabilizing and saving the damaged aircraft. 


\section{DEDICATION}

To my Mother, who has been through the challenges of life to raise and educate her children! 


\section{ACKNOWLEDGEMENTS}

First and foremost, I would like to express my sincere gratitude to Dr. Kamran Turkoglu, my advisor and mentor, whose guidance and knowledge have directed me throughout my research achievements. His dedication and sacrifices for the profession and the Flight Control Systems (FCS) and UAV Laboratory are well respected and highly appreciated.

Second, I would like to thank Dr. Nikos Mourtos from the Department of Aerospace Engineering and Dr. Ping Hsu from the Department of Electrical Engineering, who have spent their precious time to serve on my thesis committee. Discussions with them have helped me broaden my technical and intellectual perspectives.

Third, I would like to thank Dr. Fei Sun, the post-doctoral research fellow at the Flight Control Systems (FCS) and UAV Laboratory, for her help during my research at the FCS Laboratory.

Fourth, I would like to thank the Department of Aerospace Engineering faculty and staff for their support throughout my undergraduate and graduate degree pursuits.

Last but definitely not least, I would like to thank my Mother and Sister,

whose unconditional love and care have been encouraging me every single day to better myself as a person. 
LIST OF TABLES..........................................................................................................................

LIST OF FIGURES..................................................................................................

NOMENCLATURE .........................................................................................................................

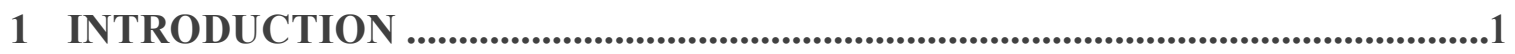

2 PROBLEM STATEMENT AND THESIS OUTLINE ..................................................3

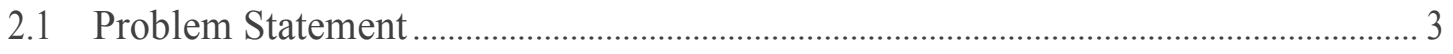

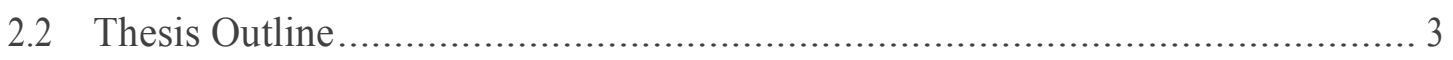

3 THE AIRCRAFT MODELS AND DATA ............................................................. 5

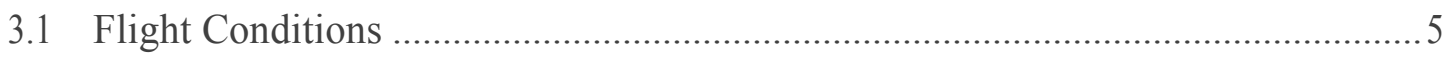

3.2 The Aircraft Models......................................................................................

3.2.1 The Nominal Aircraft Model..................................................................

3.2.2 The Damaged Aircraft Model.......................................................................

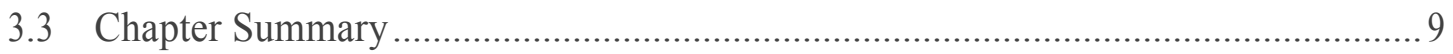

4 LATERAL/DIRECTIONAL DYNAMIC STABILITY ANALYSIS ............................10

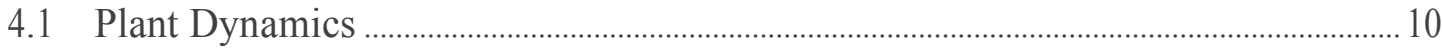

4.2 Transfer Functions ............................................................................................................................... 14

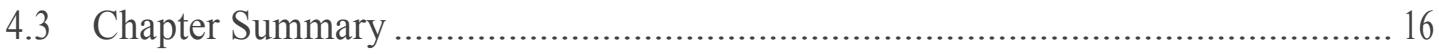

5 FREQUENCY DOMAIN ANALYSIS..............................................................................................17

5.1 Frequency Response Characteristics ...................................................................................... 17 
5.2 Robustness and Uncertainty Analysis of Frequency Response .....................................2

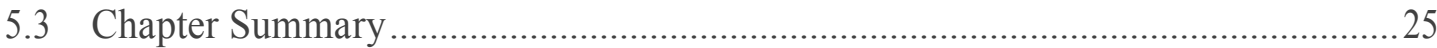

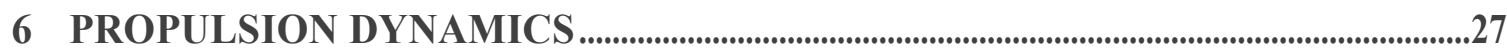

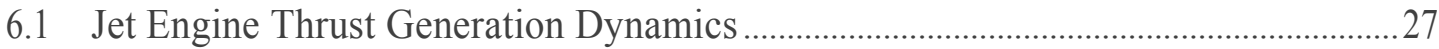

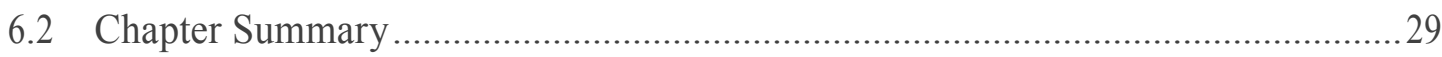

7 DIFFERENTIAL THRUST AS A CONTROL MECHANISM .........................30

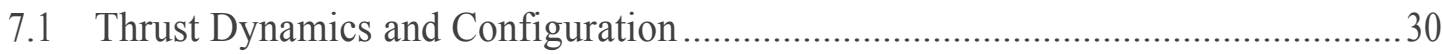

7.2 Rudder Input to Differential Thrust Input Mapping Logic ..................................30

7.3 Radian to Pound-Force Conversion Factor ...............................................................33

7.4 Commanded vs. Available Differential Thrust ......................................................... 34

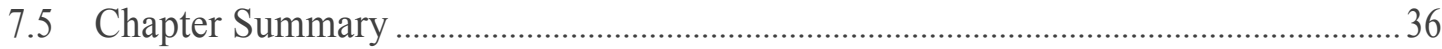

8 OPEN LOOP SYSTEM RESPONSE ANALYSIS ...........................................................

8.1 Open Loop Responses of the Damaged Aircraft ................................................ 37

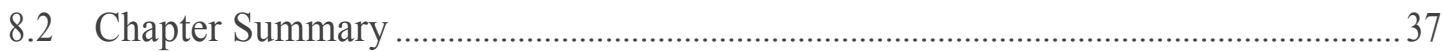

9 CONTROLLABILITY OF THE DAMAGED AIRCRAFT .................................39

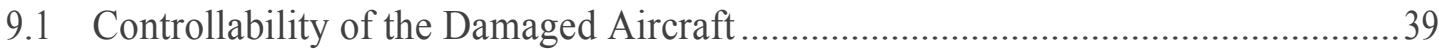

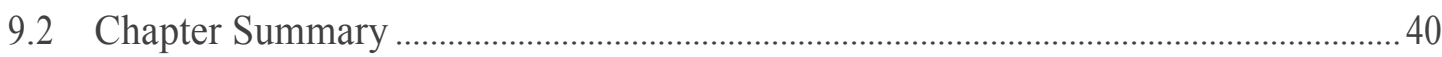

10 LINEAR QUADRATIC REGULATOR CONTROL SYSTEM DESIGN.......... 41

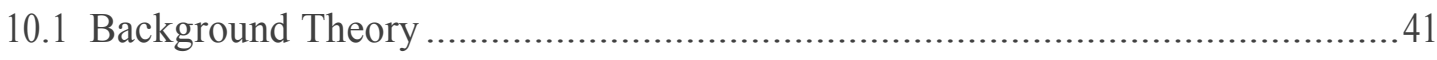

10.2 LQR Control System Design ...................................................... 44

10.3 Robustness and Sensitivity Analysis of LQR Control System .............................49 
10.3.1 Noise Sensitivity Analysis of LQR Control System.

10.3.2 Robustness and Uncertainty Analysis of LQR Control System ................56

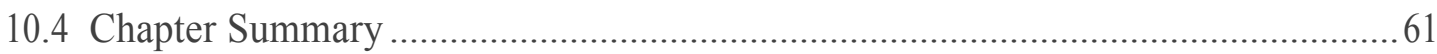

11 ADAPTIVE CONTROL SYSTEM DESIGN ..................................................62

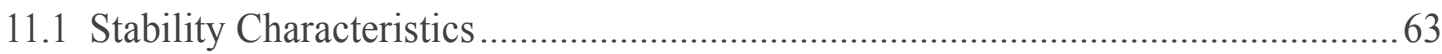

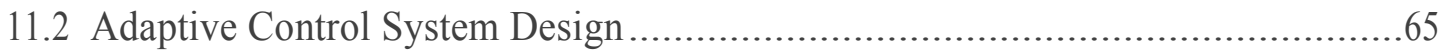

11.3 Robustness and Sensitivity Analysis of Adaptive Control System ...........................74

11.3.1 Noise Sensitivity Analysis of Adaptive Control System ...............................74

11.3.2 Robustness and Uncertainty Analysis of LQR Control System ................83

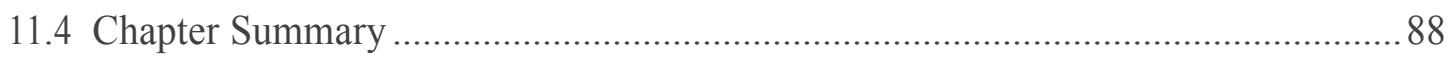

$12 H_{\infty}$ LOOP-SHAPING BASED ROBUST CONTROL SYSTEM DESIGN .........89

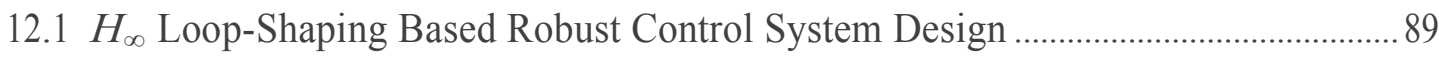

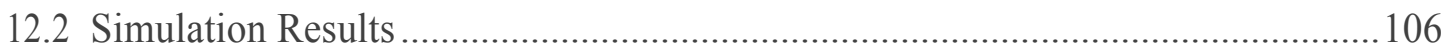

12.3 Robustness and Sensitivity Analysis of $H_{\infty}$ Loop-Shaping Based Robust Control System ......................................................................................... 110

12.3.1 Noise Sensitivity Analysis of $H_{\infty}$ Loop-Shaping Based Robust

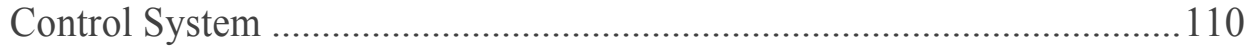

12.3.2 Robustness and Uncertainty Analysis of $H_{\infty}$ Loop-Shaping Based Robust Control System ............................................................................117

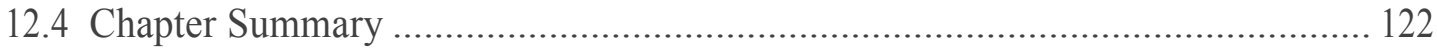

13 COMPARISON OF LQR, ADAPTIVE, AND ROBUST CONTROL METHODOLOGIES .......................................................................................................................... 123

13.1 Comparison of LQR, Adaptive, and Robust Control Methodologies....................123 


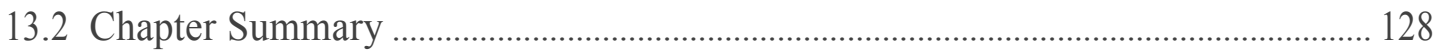

14 CONCLUSIONS AND RECOMMENDATIONS ..................................................129

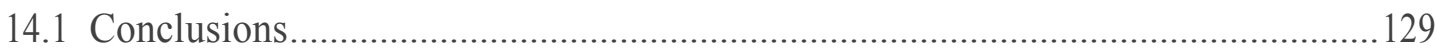

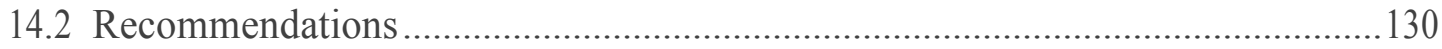

REFERENCES.............................................................................................133 


\section{LIST OF TABLES}

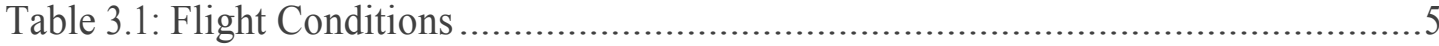

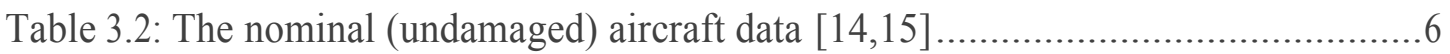

Table 3.3: The damaged aircraft mass and inertia data ..........................................

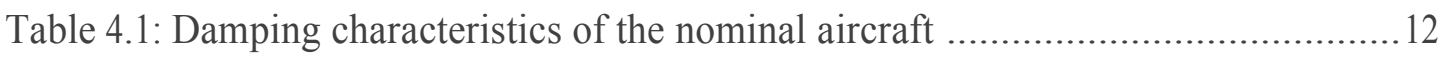

Table 4.2: Damping characteristics of the damaged aircraft ..................................... 13

Table 5.1: Open loop gain and phase margins of the damaged aircraft...........................23 


\section{LIST OF FIGURES}

Figure 4.1: Pole locations of the nominal (blue) and damaged aircraft (red)..........................13

Figure 5.1: An example of gain margin (GM) and phase margin (PM) ........................ 18

Figure 5.2: Bode diagram of the transfer function from ailerons to roll angle ...............19

Figure 5.3: Bode diagram of the transfer function from ailerons to roll rate..................19

Figure 5.4: Bode diagram of the transfer function from ailerons to side-slip angle ......20

Figure 5.5: Bode diagram of the transfer function from ailerons to yaw rate ................20

Figure 5.6: Bode diagram of the transfer function from differential thrust to roll angle 21

Figure 5.7: Bode diagram of the transfer function from differential thrust to roll rate .21

Figure 5.8: Bode diagram of the transfer function from differential thrust to side-slip angle .22

Figure 5.9: Bode diagram of the transfer function from differential thrust to yaw rate .22

Figure 5.10: Bode diagram of nominal (blue) and damaged aircraft (red) .24

Figure 5.11: Bode diagram of damaged (red) and uncertain plant (black) at $30 \%$ uncertainty

Figure 6.1: Engine thrust response at Mach 0.65 and 20,000 feet .............................28

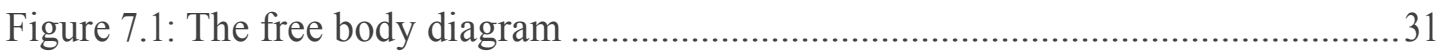

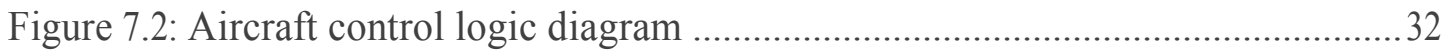

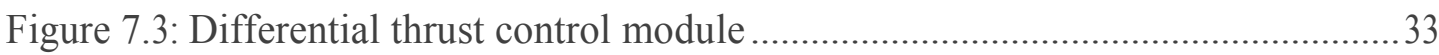

Figure 7.4: Commanded vs. available differential thrust.............................................35 


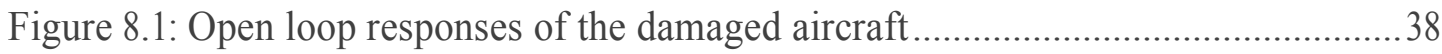

Figure 10.1: Block diagram for full-state feedback LQR control system design .............44

Figure 10.2: Closed loop responses of the damaged aircraft with an LQR controller......46

Figure 10.3: Closed loop heading angle response with an LQR controller .....................47

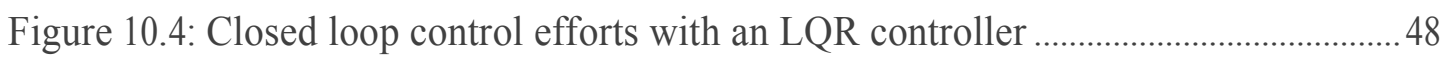

Figure 10.5: Block diagram for full-state feedback LQR control system design in

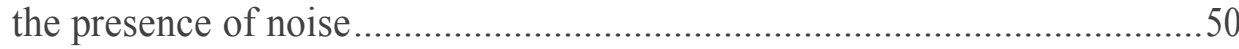

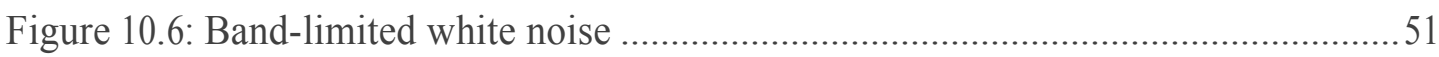

Figure 10.7: Periodogram using Fast Fourier Transform ...............................................52

Figure 10.8: Closed loop responses of the damaged aircraft with an LQR controller in the presence of noise .....................................................................................53

Figure 10.9: Closed loop heading angle response with an LQR controller in the presence

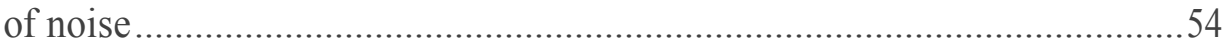

Figure 10.10: Closed loop control efforts with an LQR controller in the presence of noise .55

Figure 10.11: Block diagram for full-state feedback LQR control system design in the presence of uncertainty .......................................................................5

Figure 10.12: Closed loop responses of the damaged aircraft with an LQR controller in the presence of $30 \%$ uncertainty .

Figure 10.13: Closed loop heading angle response with an LQR controller in the presence of $30 \%$ uncertainty .58

Figure 10.14: Closed loop control efforts with an LQR controller in the presence of $30 \%$ uncertainty

Figure 11.1: Block diagram for adaptive control system design .68

Figure 11.2: Closed loop responses of the damaged aircraft with an adaptive controller. 
Figure 11.3: Closed loop heading angle response with an adaptive controller .71

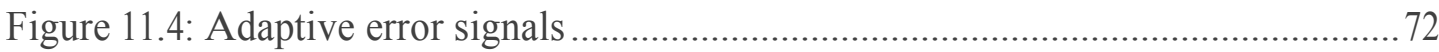

Figure 11.5: Closed loop control efforts with an adaptive controller ..............................73

Figure 11.6: Block diagram for adaptive control system design in the presence of noise. .75

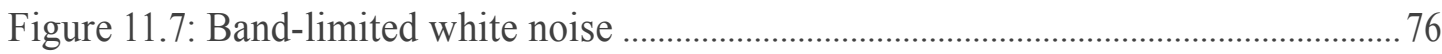

Figure 11.8: Periodogram using Fast Fourier Transform ........................................................ 77

Figure 11.9: Closed loop responses of the damaged aircraft with an adaptive controller in the presence of noise.

Figure 11.10: Closed loop heading angle response with an adaptive controller in the

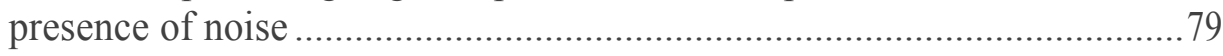

Figure 11.11: Adaptive error signals in the presence of noise ...................................... 80

Figure 11.12: Closed loop control efforts with an adaptive controller in the presence of noise

Figure 11.13: Block diagram for adaptive control system design in the presence of uncertainty

Figure 11.14: Closed loop responses of the damaged aircraft with an adaptive controller in the presence of $30 \%$ uncertainty .84

Figure 11.15: Closed loop heading angle response with an adaptive controller in the presence of $30 \%$ uncertainty .85

Figure 11.16: Adaptive error signals in the presence of $30 \%$ uncertainty .86

Figure 11.17: Closed loop control efforts with an adaptive controller in the presence of $30 \%$ uncertainty .87

Figure 12.1: The damaged aircraft's shaped plant and controller 90

Figure 12.2: Block diagram for $H_{\infty}$ loop-shaping based robust control system design .. 91

Figure 12.3: Input sensitivity function .94 


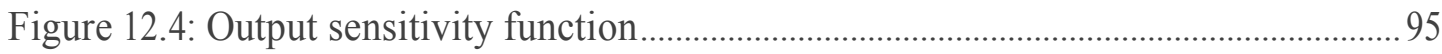

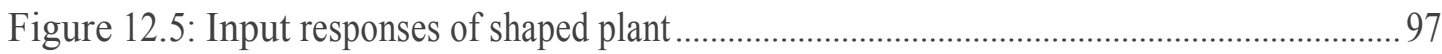

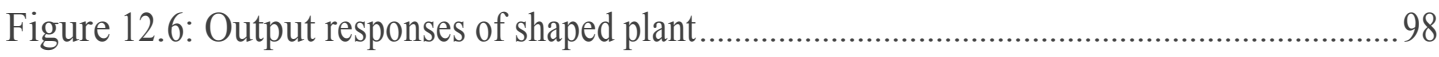

Figure 12.7: Augmented control action by the $H_{\infty}$ loop-shaping robust controller .........99

Figure 12.8: Co-sensitivity plot for input responses of shaped plant .............................100

Figure 12.9: Co-sensitivity plot for output responses of shaped plant ............................... 101

Figure 12.10: Open-loop $(\mathrm{G})$ vs. shaped $\left(\mathrm{G}_{\mathrm{s}}\right)$ vs. robustified $\left(\mathrm{G}_{\mathrm{s}} \mathrm{K}_{\mathrm{s}}\right)$ for aileron input...102

Figure 12.11: Open-loop $(G)$ vs. shaped $\left(G_{s}\right)$ vs. robustified $\left(G_{s} K_{s}\right)$ for differential

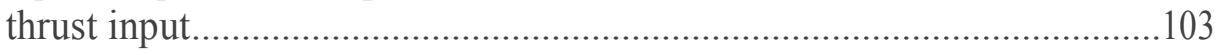

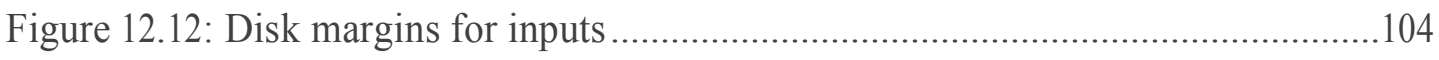

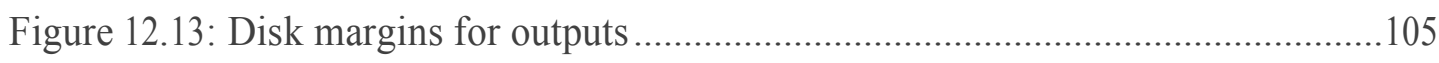

Figure 12.14: Closed loop responses of the damaged aircraft with a robust controller . 107

Figure 12.15: Closed loop heading response with a robust controller ..........................108

Figure 12.16: Closed loop control efforts with a robust controller ..................................... 109

Figure 12.17: Block diagram for $H_{\infty}$ loop-shaping based robust control system design in the presence of noise ........................................................................111

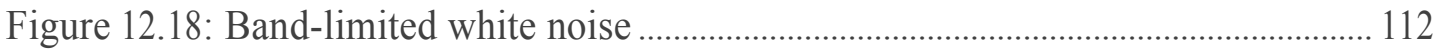

Figure 12.19: Periodogram using Fast Fourier Transform ............................................... 113

Figure 12.20: Closed loop responses of the damaged aircraft with a robust controller in the presence of noise ....................................................................... 114

Figure 12.21: Closed loop heading angle response with a robust controller in the presence of noise

Figure 12.22: Closed loop control efforts with a robust controller in the presence of noise 
Figure 12.23: Block diagram for $H_{\infty}$ loop-shaping based robust control system design in the presence of uncertainty

Figure 12.24: Closed loop responses of the damaged aircraft with a robust controller in the presence of $30 \%$ uncertainty

Figure 12.25: Closed loop heading angle response with a robust controller in the presence of $30 \%$ uncertainty

Figure 12.26: Closed loop control efforts with a robust controller in the presence of $30 \%$ uncertainty

Figure 13.1: Comparison of closed loop responses of the damaged aircraft ...................124

Figure 13.2: Comparison of closed loop heading angle responses ..................................125

Figure 13.3: Comparison of closed loop control efforts ……………………………...126 


\section{NOMENCLATURE}

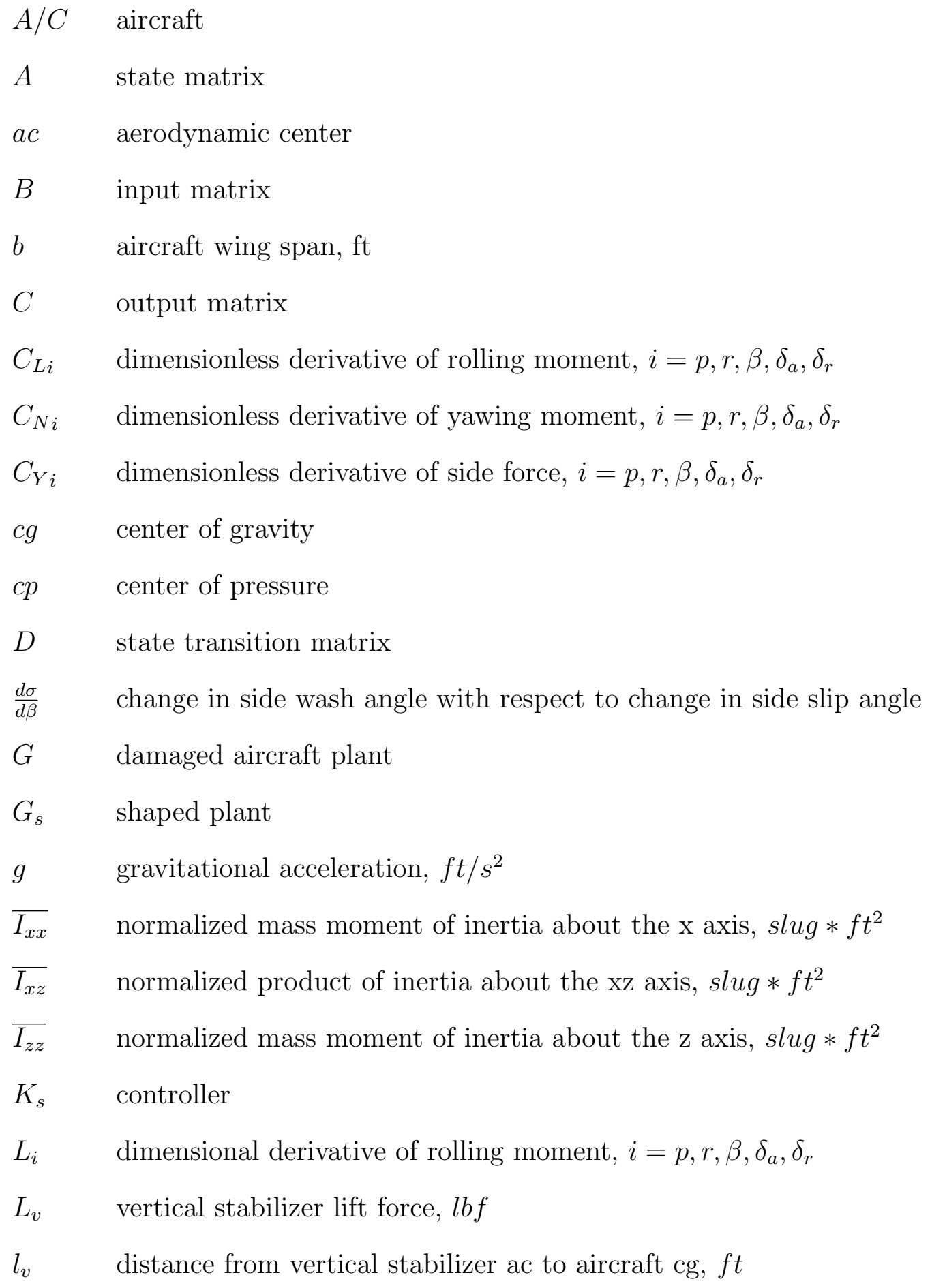


$M R A S$ model reference adaptive system

m aircraft mass, slugs

$N_{i} \quad$ dimensional derivative of yawing moment, $i=p, r, \beta, \delta_{a}, \delta_{r}, \delta T$

$p \quad$ roll rate, $\operatorname{deg} / s$

$r \quad$ yaw rate, $\mathrm{deg} / \mathrm{s}$

$S \quad$ aircraft wing area, $f t^{2}$

$S_{v} \quad$ vertical stabilizer area, $f t^{2}$

$T \quad$ engine thrust, $l b f$

$T_{c} \quad$ commanded engine thrust, $l b f$

$t \quad$ time, $s$

$t_{d} \quad$ time delay, $s$

$V_{v} \quad$ vertical stabilizer volume ratio, $f t^{3}$

$v \quad$ airspeed, $\mathrm{ft} / \mathrm{s}$

$W \quad$ aircraft weight, lbs

$W_{1} \quad$ pre-compensation

$W_{2} \quad$ post-compensation

$Y_{i} \quad$ dimensional derivative of side force, $i=p, r, \beta, \delta_{a}, \delta_{r}$

$y_{e} \quad$ distance from the outermost engine to the aircraft center of gravity, $f t$

$z_{v} \quad$ distance from the vertical stabilizer cp to the fuselage center line, $f t$

$\alpha \quad$ angle of attack, deg

$\beta \quad$ side slip angle, deg

$\gamma \quad$ flight path angle, deg

$\delta a \quad$ aileron deflection, deg

$\delta r \quad$ rudder deflection, deg

$\Delta T \quad$ collective thrust, $l b f$ 


$\begin{array}{ll}\delta T & \text { differential thrust, lbf } \\ \zeta & \text { damping ratio } \\ \eta & \text { efficiency factor } \\ \theta & \text { pitch angle, deg } \\ \rho & \text { air density, slug/ft } \\ \tau & \text { time constant, } s \\ \phi & \text { roll angle, deg } \\ \omega & \text { bandwidth frequency, } 1 / s \\ \text { ( ) } & \text { first order time derivative } \\ (\cdot & \text { second order time derivative } \\ ()^{3} & \text { trimmed value } \\ ()_{d} & \text { damaged aircraft component } \\ ()_{m} & \text { model aircraft component } \\ ()_{n} & \text { nominal (undamaged) aircraft component }\end{array}$




\section{CHAPTER 1}

\section{INTRODUCTION}

The vertical stabilizer is an essential element in providing the aircraft with its directional stability characteristic while the rudder serves as the primary control surface of yaw. In the event of an aircraft losing its vertical stabilizer, the sustained damage will cause directional stability to be compromised, and the lack of control is likely to result in a fatal crash. Notable examples of such a scenario are the crash of the American Airlines Flight 587 in 2001 when an Airbus A300-600 lost its vertical stabilizer in wake turbulence, killing all passengers and crew members [1], and the crash of Japan Airlines Flight 123 in 1985 when a Boeing 747-SR100 lost its vertical stabilizer, leading to an uncontrollable aircraft, resulting in 520 casualties [2].

However, not all situations of losing the vertical stabilizer have resulted in a total disaster. In one of those cases, United Airlines Flight 232 in 1989 [3], differential thrust was proven to be able to make the aircraft controllable. Another remarkable endeavor is the landing of the Boeing 52-H in 1964, even though the aircraft lost most of its vertical stabilizer [4].

Research on this topic has been conducted with two main goals: to understand the response characteristics of the damaged aircraft such as the work of Bacon and Gregory [5], Nguyen and Stepanyan [6], and Shah [7], as well as to come up with an automatic control algorithm to save the aircraft from disasters such as the work of Burcham et al. [8], Guo et al. [9], Liu et al. [10], Tao and Ioanou [11], and Urnes and Nielsen [12].

Notable research on the topic of a damaged transport aircraft includes the 
work of Shah [7], where a wind tunnel study was performed to evaluate the aerodynamic effects of damages to lifting and stability/control surfaces of a commercial transport aircraft. In his work, Shah [7] studied this phenomenon in the form of partial or total loss of the wing, horizontal stabilizer, or vertical stabilizer for the development of flight control systems to recover the damaged aircraft from adverse events. The work of Nguyen and Stepanyan [6] investigates the effect of the engine response time requirements of a generic transport aircraft in severe damage situations associated with the vertical stabilizer. They carried out a study which concentrated on assessing the requirements for engine design for fast responses in an emergency situation. In addition, the use of differential thrust as a propulsion command for the control of directional stability of a damaged transport aircraft was studied by Urnes and Nielsen [12] to identify the effect of the change in aircraft performance due to the loss of the vertical stabilizer and to make an improvement in stability utilizing engine thrust as an emergency yaw control mode with feedback from the aircraft motion sensors.

Existing valuable research in the literature provides very unique insight regarding the dynamics of such an extreme scenario whereas in this thesis, a unique extension of existing work is provided where automatic control methodologies to aid a damaged aircraft to land safely are implemented. This thesis is motivated to improve air travel safety by incorporating the utilization of differential thrust to regain lateral/directional stability for a commercial aircraft (in this case, a Boeing 747-100) with a damaged vertical stabilizer. 


\section{CHAPTER 2}

\section{PROBLEM STATEMENT AND THESIS OUTLINE}

\subsection{Problem Statement}

For this thesis, the flight scenario is chosen to be a steady, level cruise flight for the Boeing 747-100 at Mach 0.65 and 20,000 feet. At one point during the flight, as a result of external disturbances such as wake turbulence, the vertical stabilizer of the aircraft is completely damaged. In such an extreme scenario, it is desired to develop and implement differential thrust based control strategies to stabilize and save the damaged aircraft.

\section{$2.2 \quad$ Thesis Outline}

In order to eliminate the dangerous coupling between the aircraft and pilots, which usually leads to a total disaster in such an extreme scenario, the following methodology is followed to establish a strategy that will save the damaged aircraft:

(1) The nominal and damaged aircraft models are constructed in Chapter 3.

(2) Lateral/directional dynamic stability analysis is conducted in Chapter 4.

(3) Frequency domain analysis is carried out in Chapter 5.

(4) The engine dynamics of the jet aircraft are modeled as a system of differential equations with corresponding time constant and time delay terms to study the engine response characteristics with respect to a differential thrust input in Chapter 6 . 
(5) The novel differential thrust control module is developed to map a rudder input to a differential thrust input in Chapter 7.

(6) The aircraft's open loop responses are investigated in Chapter 8.

(7) The controllability of the damaged aircraft is investigated in Chapter 9.

(8) The linear quadratic regulator (LQR) controller is designed to stabilize the damaged aircraft in Chapter 10.

(9) The Lyapunov stability approach based model reference adaptive control methodology is implemented to test the ability of the damaged aircraft to mimic the model aircraft's reference responses and achieve safe and stable operating conditions in Chapter 11.

(10) The robust control system design based on $H_{\infty}$ loop-shaping approach is implemented as a means to stabilize the damaged aircraft in Chapter 12. It is also worth noting that for each control methodology such as the linear quadratic regulator (LQR), model reference adaptive system (MRAS), and $H_{\infty}$ loop-shaping based robust control system, the robustness and sensitivity analysis is conducted to test the performance and overall robustness of each control system in the presence of noise and uncertainty.

(11) A comparison study of the three control systems is conducted to investigate the merits and limits of each control system to save the damaged aircraft in Chapter 13.

(12) Conclusions and recommendations are in Chapter 14. 


\section{CHAPTER 3}

\section{THE AIRCRAFT MODELS AND DATA}

Here, nominal (undamaged) and damaged aircraft models are developed for analysis.

\section{1 $\quad$ Flight Conditions}

The flight conditions for both the damaged and undamaged aircraft models in this thesis are summarized in Table 3.1.

Table 3.1: Flight conditions

\begin{tabular}{|c|c|}
\hline Parameter & Value \\
\hline Altitude $(f t)$ & 20,000 \\
\hline Air Density $\left(\right.$ slugs $\left./ \mathrm{ft}^{3}\right)$ & 0.001268 \\
\hline Airspeed $(\mathrm{ft} / \mathrm{s})$ & 673 \\
\hline
\end{tabular}

\subsection{The Aircraft Models}

\subsubsection{The Nominal Aircraft Model}

The Boeing 747-100 was chosen for this thesis due to its widely available technical specifications, aerodynamics, and stability derivative data. The data for the nominal (undamaged) Boeing 747-100 are summarized in Table 3.2.

Taken from Nguyen and Stepanyan [6], the lateral/directional linear equations of motion of the nominal (undamaged) aircraft, with the intact ailerons and rudder as control inputs, are presented in Eq. (3.1). 
Table 3.2: The nominal (undamaged) aircraft data $[14,15]$

\begin{tabular}{|c|c|}
\hline Parameter & Value \\
\hline$S\left(f t^{2}\right)$ & 5500 \\
\hline$b(f t)$ & 196 \\
\hline $\bar{c}(f t)$ & 27.3 \\
\hline$y_{e}(f t)$ & 69.83 \\
\hline$W(l b s)$ & $6.3663 * 10^{5}$ \\
\hline$m(s l u g s)$ & 19786.46 \\
\hline$I_{x x}\left(s l u g * f t^{2}\right)$ & $18.2 * 10^{6}$ \\
\hline$I_{y y}\left(s l u g * f t^{2}\right)$ & $33.1 * 10^{6}$ \\
\hline$I_{z z}\left(s l u g * f t^{2}\right)$ & $49.7 * 10^{6}$ \\
\hline$I_{x z}\left(s l u g * f t^{2}\right)$ & $0.97 * 10^{6}$ \\
\hline$C_{L \beta}$ & -0.160 \\
\hline$C_{L p}$ & -0.340 \\
\hline$C_{L r}$ & 0.130 \\
\hline$C_{L \delta_{a}}$ & 0.013 \\
\hline$C_{L \delta_{r}}$ & 0.008 \\
\hline$C_{N \beta}$ & 0.160 \\
\hline$C_{N p}$ & -0.026 \\
\hline$C_{N r}$ & -0.28 \\
\hline$C_{N \delta_{a}}$ & 0.0018 \\
\hline$C_{N \delta_{r}}$ & -0.100 \\
\hline$C_{Y \beta}$ & -0.90 \\
\hline$C_{Y p}$ & 0 \\
\hline$C_{Y r}$ & 0 \\
\hline$C_{Y \delta_{a}}$ & 0 \\
\hline$C_{Y \delta_{r}}$ & 0.120 \\
\hline & \\
\hline & \\
\hline & \\
\hline & \\
\hline & \\
\hline & \\
\hline & \\
\hline & \\
\hline & \\
\hline & \\
\hline
\end{tabular}




$$
\left[\begin{array}{c}
\dot{\phi} \\
\dot{p} \\
\dot{\beta} \\
\dot{r}
\end{array}\right]=\left[\begin{array}{cccc}
0 & 1 & 0 & \bar{\theta} \\
0 & L_{p} & L_{\beta} & L_{r} \\
\frac{g}{V} & \frac{Y_{p}}{\bar{V}} & \frac{Y_{\beta}+g \bar{\gamma}}{\bar{V}} & \frac{Y_{p}}{\bar{V}}-1 \\
0 & N_{p} & N_{\beta} & N_{r}
\end{array}\right]\left[\begin{array}{c}
\phi \\
p \\
\beta \\
r
\end{array}\right]+\left[\begin{array}{cc}
0 & 0 \\
L_{\delta_{a}} & L_{\delta_{r}} \\
\frac{Y_{\delta_{a}}}{\bar{V}} & \frac{Y_{\delta_{r}}}{\bar{V}} \\
N_{\delta_{a}} & N_{\delta_{r}}
\end{array}\right]\left[\begin{array}{c}
\delta_{a} \\
\delta_{r}
\end{array}\right]
$$

where the states are $\phi, p, \beta$, and $r$, which represent the roll angle, roll rate, side-slip angle, and yaw rate, respectively. The corresponding control inputs are $\delta_{a}$ (aileron input) and $\delta_{r}$ (rudder input).

\subsubsection{The Damaged Aircraft Model}

For the modeling of the damaged aircraft, in case of the loss of the vertical stabilizer, lateral/directional stability derivatives need to be reexamined and recalculated. Since the whole aerodynamic structure is affected, the new corresponding stability derivatives have to be calculated and studied. The lateral/directional dimensionless derivatives that depend on the vertical stabilizer include $[16]$ :

$$
\begin{gathered}
C_{Y \beta}=-\eta \frac{S_{v}}{S} C_{L \alpha v}\left(1+\frac{d \sigma}{d \beta}\right) \\
C_{Y r}=-2\left(\frac{l_{v}}{b}\right) C_{Y \beta_{t a i l}} \\
C_{N \beta}=C_{N \beta w f}+\eta_{v} V_{v} C_{L \alpha v}\left(1+\frac{d \sigma}{d \beta}\right) \\
C_{N r}=-2 \eta_{v} V_{v}\left(\frac{l_{v}}{b}\right) C_{L \alpha v}
\end{gathered}
$$




$$
C_{L r}=\frac{C_{L}}{4}-2\left(\frac{l_{v}}{b}\right)\left(\frac{z_{v}}{b}\right) C_{Y \beta_{t a i l}}
$$

Due to the loss of the vertical stabilizer, the vertical tail area, volume, and efficiency factor will all be zero; therefore, $C_{Y \beta}=C_{Y r}=C_{N r}=0$. If the vertical stabilizer is assumed to be the primary aerodynamic surface responsible for the weathercock stability, then $C_{N \beta}=0$. Finally, $C_{L r}=\frac{C_{L}}{4}$.

In addition, without the vertical stabilizer, the mass and inertia data of the damaged aircraft are going to change, where the values that reflect such a scenario (for the damaged aircraft) are listed in Table 3.3.

Table 3.3: The damaged aircraft mass and inertia data

\begin{tabular}{|c|c|}
\hline Parameter & Value \\
\hline$W($ lbs $)$ & $6.2954 * 10^{5}$ \\
\hline$m($ slugs $)$ & 19566.10 \\
\hline$I_{x x}\left(\operatorname{slug} * f t^{2}\right)$ & $17.893 * 10^{6}$ \\
\hline$I_{y y}\left(\operatorname{slug} * f t^{2}\right)$ & $30.925 * 10^{6}$ \\
\hline$I_{z z}\left(\operatorname{slug} * f t^{2}\right)$ & $47.352 * 10^{6}$ \\
\hline$I_{x z}\left(\operatorname{slug} * f t^{2}\right)$ & $0.3736 * 10^{6}$ \\
\hline
\end{tabular}

In this study, during the loss of the vertical stabilizer, it is proposed that the differential thrust component of aircraft dynamics be utilized as an alternate control input replacing the rudder control to regain stability and control of lateral/directional flight dynamics. Next, the lateral-directional linear equations of motion of the damaged aircraft are presented, with the ailerons $\left(\delta_{a}\right)$, differential thrust $(\delta T)$, and collective thrust $(\Delta T)$ as control inputs [6], as presented in Eq. (3.7). 


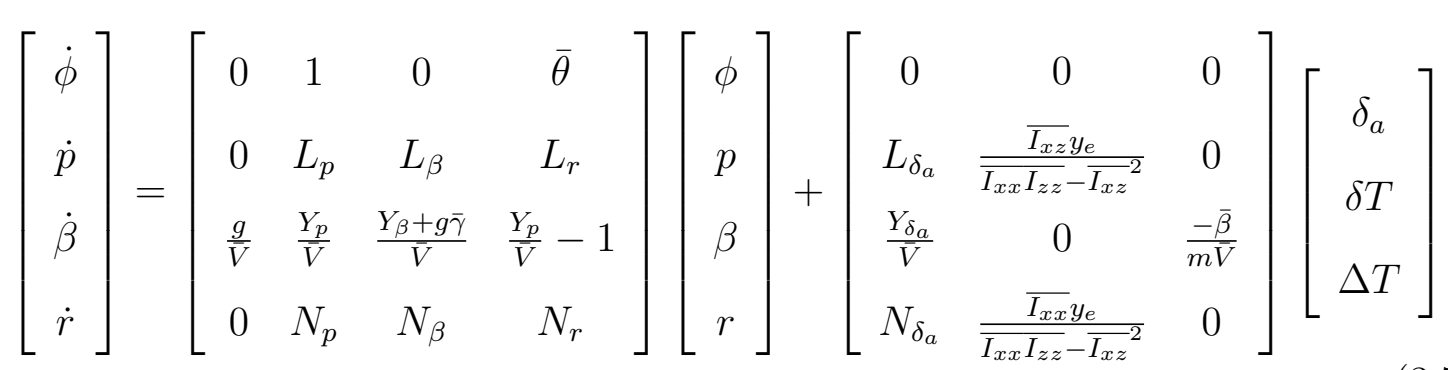

In this case, if the initial trim side-slip angle is zero, then $\Delta T$ does not have any significance in the control effectiveness for a small perturbation around the trim condition [6], which means that the above equations of motion can be reduced to the final form of governing equations of motion for damaged aircraft as presented in Eq. (3.8).

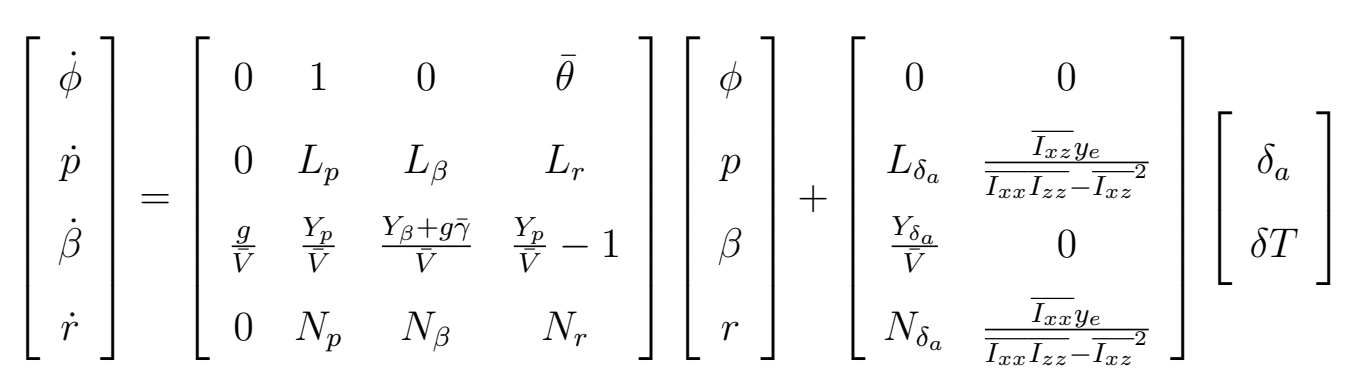

\subsection{Chapter Summary}

In this chapter, we constructed the nominal and damaged aircraft models, which are of great importance to the stability analysis and controller design phases in the following chapters. In the next chapter, we are going to conduct the lateral/directional dynamic stability analysis of the damaged aircraft. 


\section{CHAPTER 4}

\section{LATERAL/DIRECTIONAL DYNAMIC STABILITY ANALYSIS}

\subsection{Plant Dynamics}

With data taken from Table 3.1 and Table 3.2, the A, B, C, D state space matrices for the lateral/directional dynamics of the nominal (undamaged) Boeing 747-100 can be obtained as shown in Eq. (4.1-4.4).

$$
\begin{gathered}
A_{n}=\left[\begin{array}{cccc}
0 & 1 & 0 & 0 \\
0 & -0.8566 & -2.7681 & 0.3275 \\
0.0478 & 0 & -0.1079 & -1 \\
0 & -0.0248 & 1.0460 & -0.2665
\end{array}\right] \\
B_{n}=\left[\begin{array}{ccc}
0 & 0 \\
0.2249 & 0.1384 \\
0 & 0.0144 \\
0.0118 & -0.6537
\end{array}\right] \\
C_{n}=\left[\begin{array}{cccc}
1 & 0 & 0 & 0 \\
0 & 1 & 0 & 0 \\
0 & 0 & 1 & 0 \\
0 & 0 & 0 & 1
\end{array}\right]
\end{gathered}
$$




$$
D_{n}=\left[\begin{array}{ll}
0 & 0 \\
0 & 0 \\
0 & 0 \\
0 & 0
\end{array}\right]
$$

Based on the data for the lateral/directional stability derivatives of the aircraft without its vertical stabilizer given in Chapter 3.2.2, the lateral/directional A, B, C, D state space matrices for the damaged aircraft can be achieved as shown in Eq. (4.5-4.8).

$$
\begin{gathered}
A_{d}=\left[\begin{array}{cccc}
0 & 1 & 0 & 0 \\
0 & -0.8566 & -2.7681 & 0.1008 \\
0.0478 & 0 & 0 & -1 \\
0 & -0.0248 & 0 & 0
\end{array}\right] \\
B_{d}=\left[\begin{array}{ccc}
0 & 0 \\
0.2249 & 0.0142 \\
0 & 0 & \\
0.0118 & 0.6784
\end{array}\right] \\
C_{d}=C_{n}=\left[\begin{array}{cccc}
1 & 0 & 0 & 0 \\
0 & 1 & 0 & 0 \\
0 & 0 & 1 & 0 \\
0 & 0 & 0 & 1
\end{array}\right]
\end{gathered}
$$




$$
D_{d}=D_{n}=\left[\begin{array}{ll}
0 & 0 \\
0 & 0 \\
0 & 0 \\
0 & 0
\end{array}\right]
$$

Here, $A_{n}$ defines the state matrix of the nominal (undamaged) aircraft whereas $A_{d}$ represents the state matrix of the damaged aircraft. Furthermore, $B_{n}$ represents the input matrix where the ailerons $\left(\delta_{a}\right)$ and rudder $\left(\delta_{r}\right)$ are control inputs of the undamaged (nominal) aircraft, whereas $B_{d}$ stands for the input matrix of the scenario where the ailerons $\left(\delta_{a}\right)$ and differential thrust $(\delta T)$ are control inputs of the damaged aircraft. It is also worth noting that the structure of the input matrix of the nominal aircraft $\left(B_{n}\right)$ and of damaged aircraft $\left(B_{d}\right)$ remain fairly similar, except for the $b_{32}$ term, which maps the rudder input to side-slip angle output for the nominal (undamaged aircraft) and the differential input to side-slip angle output for the damaged aircraft. This is important because it signifies the dramatic consequence of losing the vertical stabilizer. In $B_{n}, b_{32}$ equals 0.0144 . However, $b_{32}$ equals 0 in $B_{d}$, which removes the direct effect of differential thrust on side-slip angle for the damaged aircraft.

In addition, the damping characteristics of the nominal and damaged aircraft are summarized in Table 4.1 and Table 4.2 .

Table 4.1: Damping characteristics of the nominal aircraft

\begin{tabular}{|c|c|c|c|c|}
\hline Mode & Pole Location & Damping & Frequency $(1 / s)$ & Period $(s)$ \\
\hline Dutch Roll & $-0.126 \pm i 1.06$ & 0.118 & 1.07 & 5.8822 \\
\hline Spiral & -0.0172 & 1 & 0.0172 & 365.2651 \\
\hline Roll & -0.963 & 1 & 0.963 & 6.5262 \\
\hline
\end{tabular}

Table 4.1 shows that all three lateral/directional modes of the nominal aircraft 
Table 4.2: Damping characteristics of the damaged aircraft

\begin{tabular}{|c|c|c|c|c|}
\hline Mode & Pole Location & Damping & Frequency $(1 / s)$ & Period $(s)$ \\
\hline Dutch Roll & $0.0917 \pm i 0.43$ & -0.209 & 0.439 & 14.2969 \\
\hline Spiral & $6.32 * 10^{-18}$ & -1 & $6.32 * 10^{-18}$ & $9.9486 * 10^{17}$ \\
\hline Roll & -1.04 & 1 & 1.04 & 6.0422 \\
\hline
\end{tabular}

are stable due to the left half plane (LHP) pole locations whereas Table 4.2 clearly indicates the unstable nature of the damaged aircraft in the Dutch roll mode by the right half plane (RHP) pole locations. Furthermore, the pole of the spiral mode lies at the origin, which represents very slow (also unstable) dynamics. The only stable mode of the damaged aircraft is the roll mode by the left half plane (LHP) pole location. The pole locations of both the nominal and damaged aircraft can also be illustrated by Fig. 4.1.

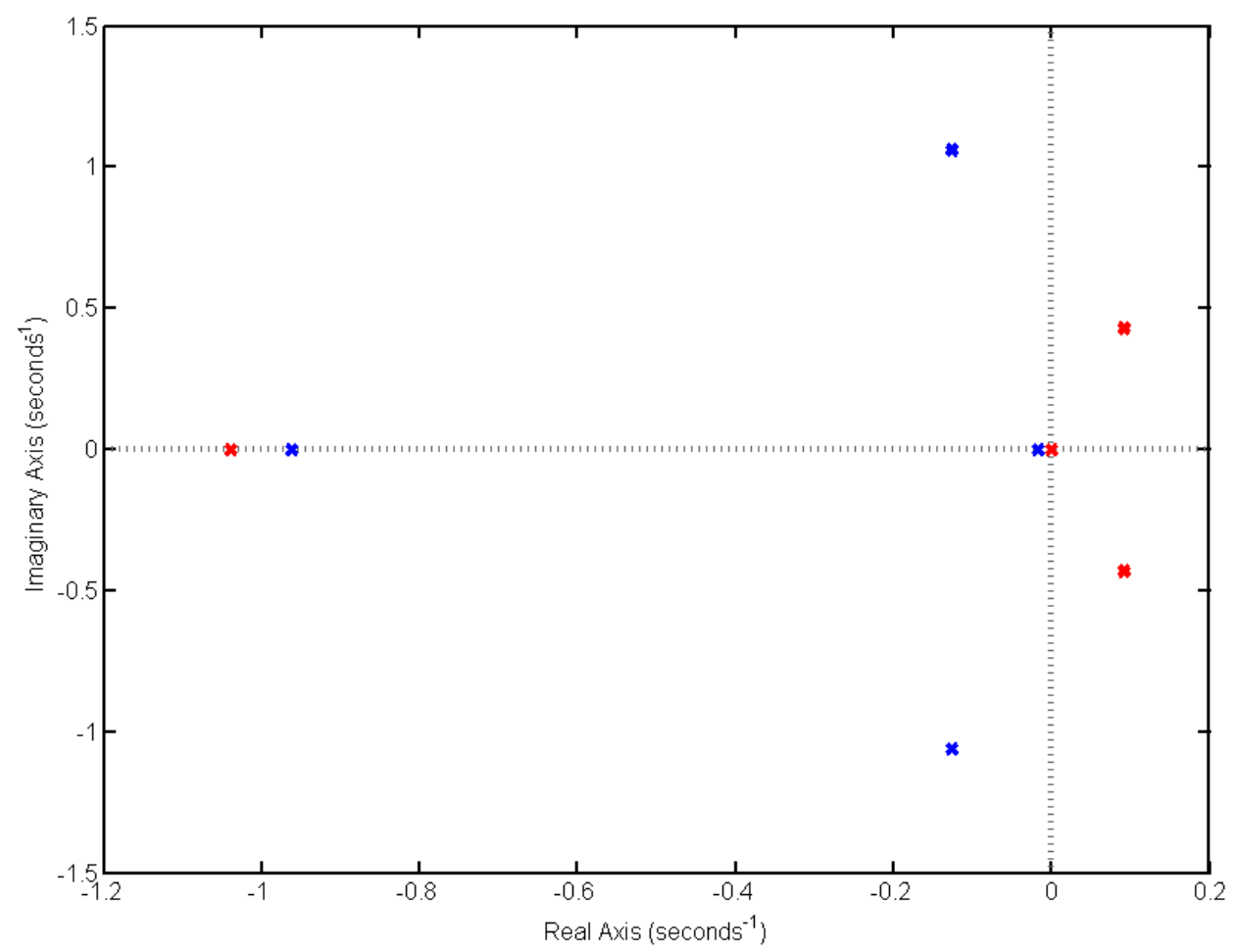

Figure 4.1: Pole locations of the nominal (blue) and damaged aircraft (red) 


\subsection{Transfer Functions}

The lateral/directional transfer functions (TF) for the damaged aircraft from each of the inputs (ailerons $\left(\delta_{a}\right)$ and differential thrust $(\delta T)$ ) to each of the outputs (roll angle $(\phi)$, roll rate $(p)$, side-slip angle $(\beta)$, and yaw rate $(r)$ ) are obtained and analyzed.

For a continuous time state space system represented as in Eq. (4.9)

$$
\begin{aligned}
& \dot{x}=A x+B u \\
& y=C x+D u
\end{aligned}
$$

with non-zero initial conditions, the Laplace transform of Eq. (4.9) is represented in Eq. (4.10).

$$
\begin{array}{r}
s X(s)-X(0)=A X(s)+B U(s) \\
Y(s)=C X(s)+D U(s)
\end{array}
$$

which will then lead to

$$
X(s)(s I-A)=X(0)+B U(s)
$$

or

$$
X(s)=(s I-A)^{-1}[X(0)+B U(s)]
$$

Therefore,

$$
Y(s)=C(s I-A)^{-1}[X(0)+B U(s)]+D U(s)
$$

With zero initial conditions, Eq. (4.13) can be written as 


$$
Y(s)=\left[C(s I-A)^{-1} B+D\right] U(s)
$$

Finally, one can use Eq. (4.15) to find the transfer functions of a system with zero initial conditions.

$$
\frac{Y(s)}{U(s)}=C(s I-A)^{-1} B+D
$$

Here the state-transmission matrix, $D$, is zero assuming there is no direct effect on the outputs from the inputs. Therefore,

$$
\frac{Y(s)}{U(s)}=C(s I-A)^{-1} B
$$

With the information of the lateral/directional matrices for the damaged aircraft from Chapter 4.1, the transfer functions from each of the inputs (ailerons $\left(\delta_{a}\right)$ and differential thrust $\left.(\delta T)\right)$ to each of the outputs (roll angle $(\phi)$, roll rate $(p)$, side-slip angle $(\beta)$, and yaw rate $(r))$ can be obtained as in Eq. (4.17-4.24).

$$
\begin{gathered}
\frac{\phi(s)}{\delta_{a}(s)}=\frac{0.22491\left(s^{2}+0.005272 s+0.1448\right)}{s(s+1.04)\left(s^{2}-0.1833 s+0.1931\right)} \\
\frac{p(s)}{\delta_{a}(s)}=\frac{0.22491 s\left(s^{2}+0.005272 s+0.1448\right)}{s(s+1.04)\left(s^{2}-0.1833 s+0.1931\right)} \\
\frac{\beta(s)}{\delta_{a}(s)}=\frac{-0.011767(s-0.5392)(s+0.008935)}{s(s+1.04)\left(s^{2}-0.1833 s+0.1931\right)} \\
\frac{r(s)}{\delta_{a}(s)}=\frac{0.011767(s+0.6744)\left(s^{2}-0.2909 s+0.1962\right)}{s(s+1.04)\left(s^{2}-0.1833 s+0.1931\right)} \\
\frac{\phi(s)}{\delta T(s)}=\frac{0.014166\left(s^{2}+4.826 s+132.6\right)}{s(s+1.04)\left(s^{2}-0.1833 s+0.1931\right)}
\end{gathered}
$$




$$
\begin{gathered}
\frac{p(s)}{\delta T(s)}=\frac{0.014166 s\left(s^{2}+4.826 s+132.6\right)}{s(s+1.04)\left(s^{2}-0.1833 s+0.1931\right)} \\
\frac{\beta(s)}{\delta T(s)}=\frac{-0.67841(s+0.8606)(s-0.005598)}{s(s+1.04)\left(s^{2}-0.1833 s+0.1931\right)} \\
\frac{r(s)}{\delta T(s)}=\frac{0.67841(s+0.9908)\left(s^{2}-0.1348 s+0.1336\right)}{s(s+1.04)\left(s^{2}-0.1833 s+0.1931\right)}
\end{gathered}
$$

From the transfer functions from ailerons and differential thrust to roll rate, Eq. (4.18) and Eq. (4.22), it can be seen that there might be the pole-zero cancellation effect of the pole and zero at the origin. This pole-zero cancellation effect may reduce the order of the system and result in dramatic change in the response characteristics of the system itself. Therefore, the pole-zero cancellation is not conducted to reserve the response characteristics of the original system.

\subsection{Chapter Summary}

In this chapter, we set up the A, B, C, D state space matrices for both the nominal (undamaged) and damaged aircraft models. We also looked at the damping characteristics of both the undamaged and damaged aircraft. Additionally, the transfer functions from the aileron $\left(\delta_{a}\right)$ and differential thrust $(\delta T)$ inputs to the roll angle $(\phi)$, roll rate $(p)$, side-slip angle $(\beta)$, and yaw rate $(r)$ outputs were obtained. In the next chapter, we are conducting the frequency domain analysis of the damaged aircraft and comparing it to the nominal (undamaged) aircraft's. 


\section{CHAPTER 5}

\section{FREQUENCY DOMAIN ANALYSIS}

\subsection{Frequency Response Characteristics}

In this chapter, the important aspects of frequency domain analysis such as gain margin (GM) and phase margin (PM) are discussed. GM is defined as the amount of gain variation which can be tolerated before the system reaches instability. GM corresponds to the amount of gain at -180 degree crossover at phase. PM is defined as the amount of phase variation which can be tolerated before the system reaches instability. PM corresponds to the amount of phase at 0 dB crossover at gain. Together, GM and PM provide the estimated stability safety margins. The higher the margins are, the more stable the system is, and vice versa,

which represents the trade-off between stability and maneuverability. An example of GM and PM is shown in Fig. 5.1.

Furthermore, the Bode diagrams of the transfer functions from each of the inputs (ailerons $\left(\delta_{a}\right)$ and differential thrust $(\delta T)$ ) to each of the outputs (roll angle $(\phi)$, roll rate $(p)$, side-slip angle $(\beta)$, and yaw rate $(r))$ of the damaged aircraft are shown in Fig. 5.2-5.9. From Fig. 5.2 and Fig. 5.3, we can see that there exist dips in the magnitude plots of the Bode diagrams. Also, in Fig. 5.9, there is also a dip in the phase plot of the Bode diagrams. This happens due to the existence of complex conjugate right half plane (RHP) zeros in the corresponding transfer functions, which creates a non-minimum phase dynamic. These dips do have a negative impact on stability and more importantly, performance. 


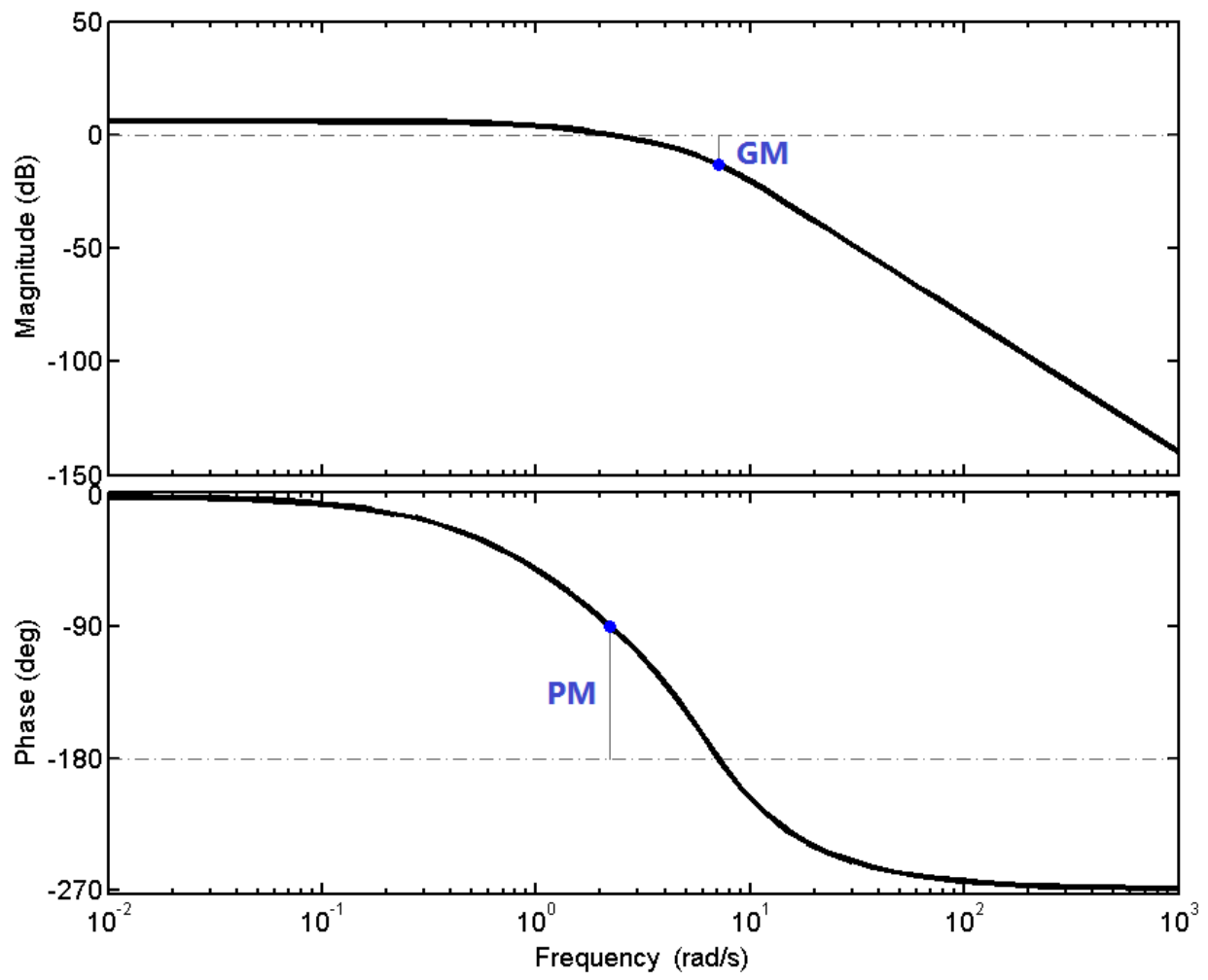

Figure 5.1: An example of gain margin (GM) and phase margin (PM) 


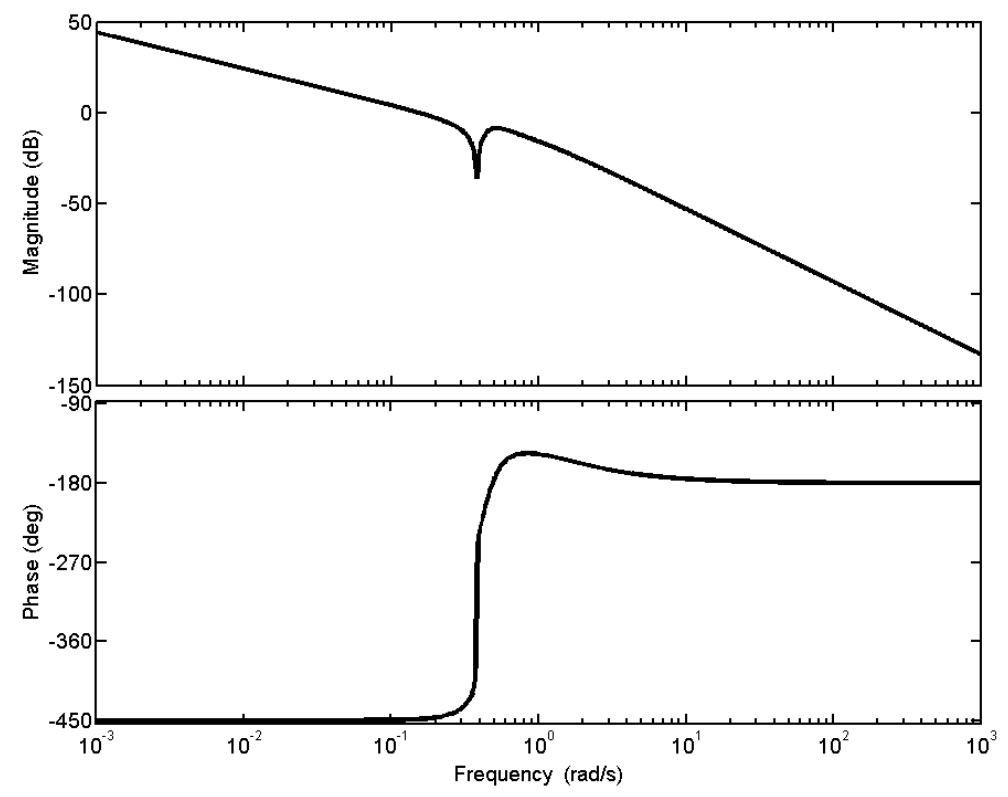

Figure 5.2: Bode diagram of the transfer function from ailerons to roll angle

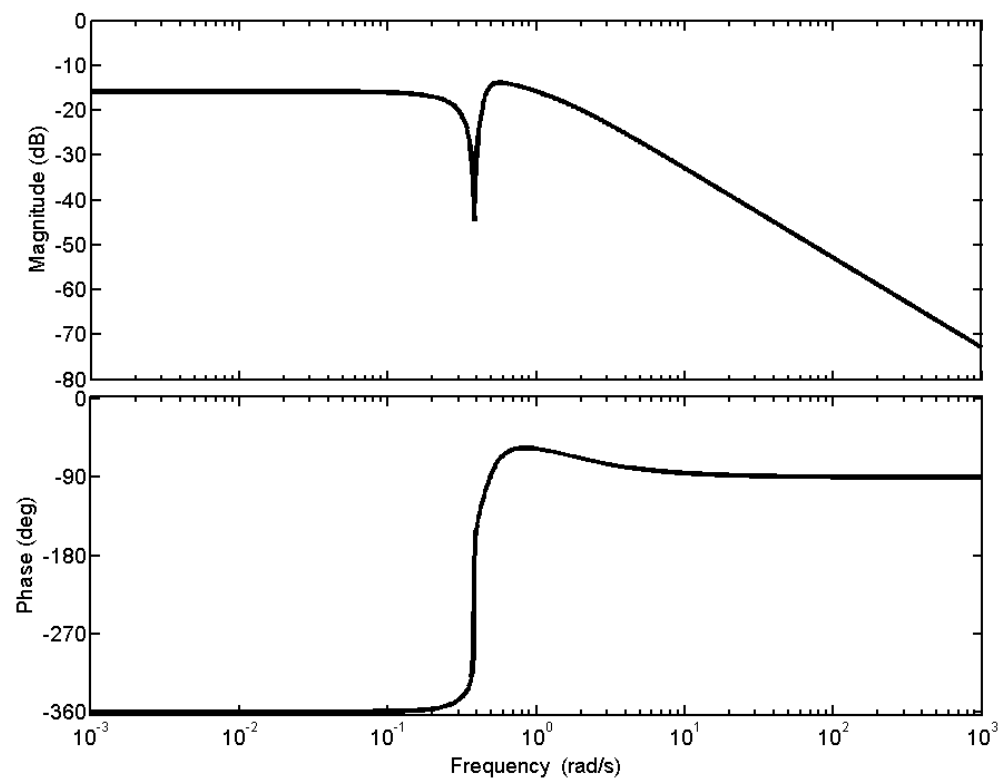

Figure 5.3: Bode diagram of the transfer function from ailerons to roll rate 


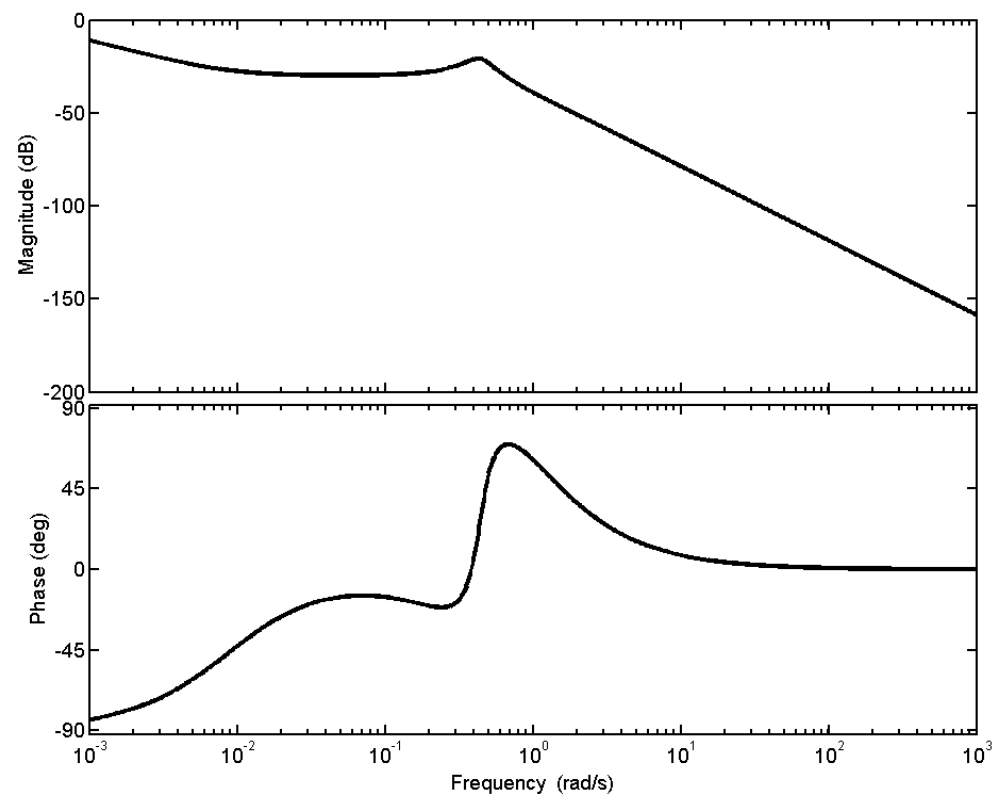

Figure 5.4: Bode diagram of the transfer function from ailerons to side-slip angle

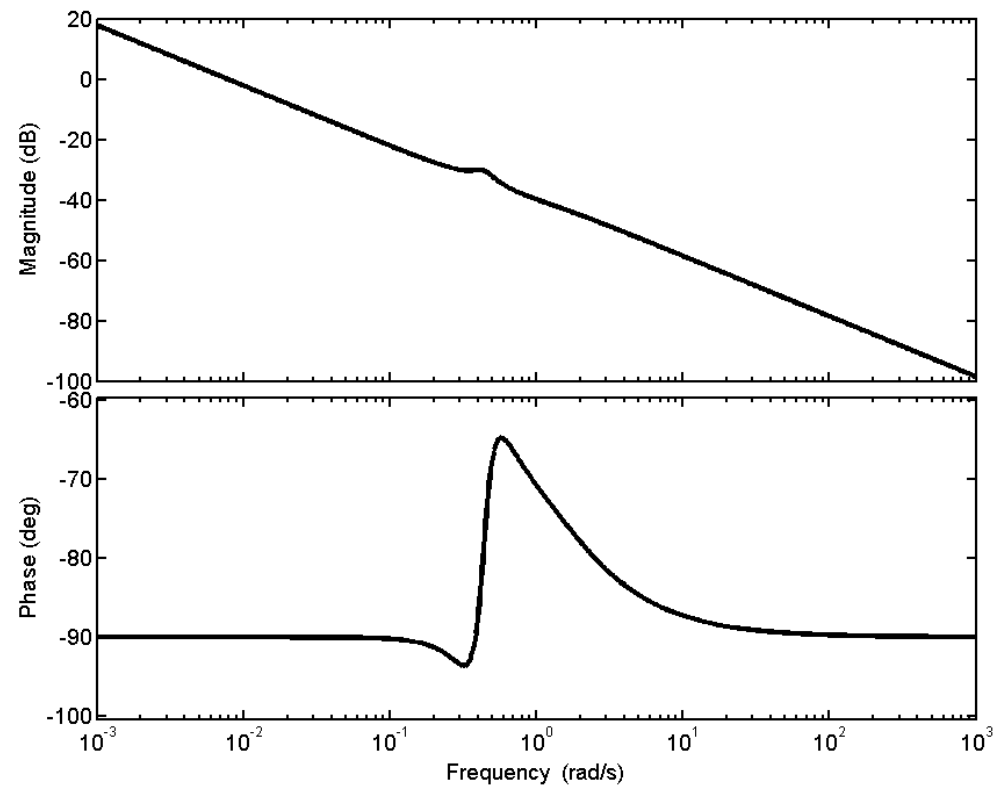

Figure 5.5: Bode diagram of the transfer function from ailerons to yaw rate 


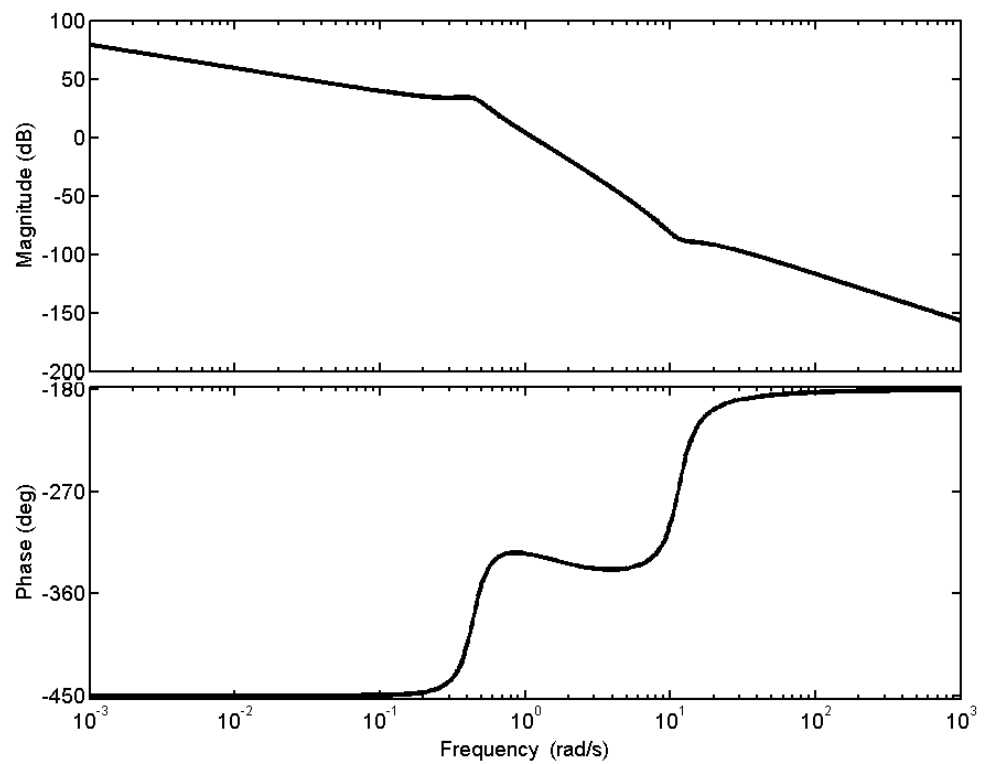

Figure 5.6: Bode diagram of the transfer function from differential thrust to roll angle

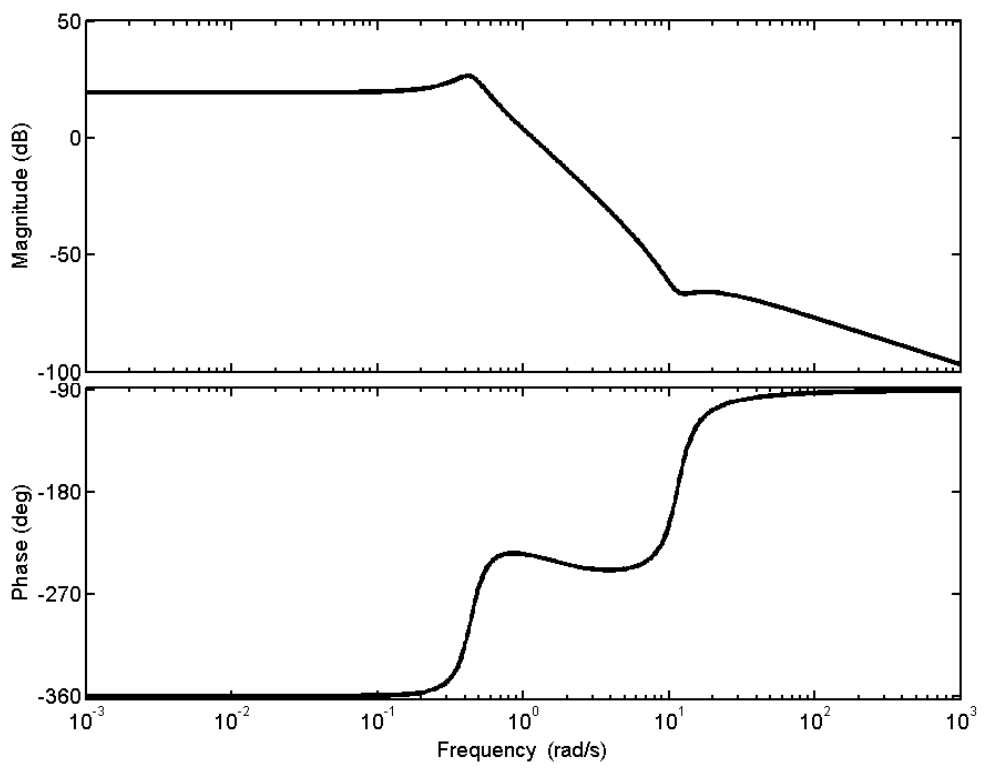

Figure 5.7: Bode diagram of the transfer function from differential thrust to roll rate 


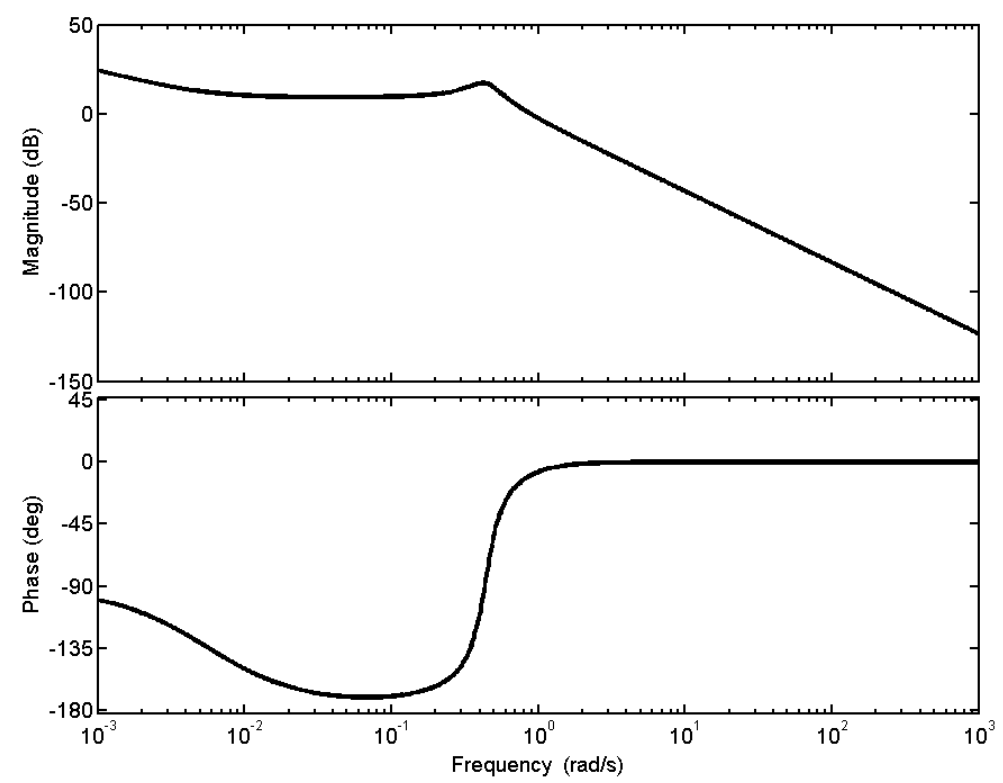

Figure 5.8: Bode diagram of the transfer function from differential thrust to side-slip angle

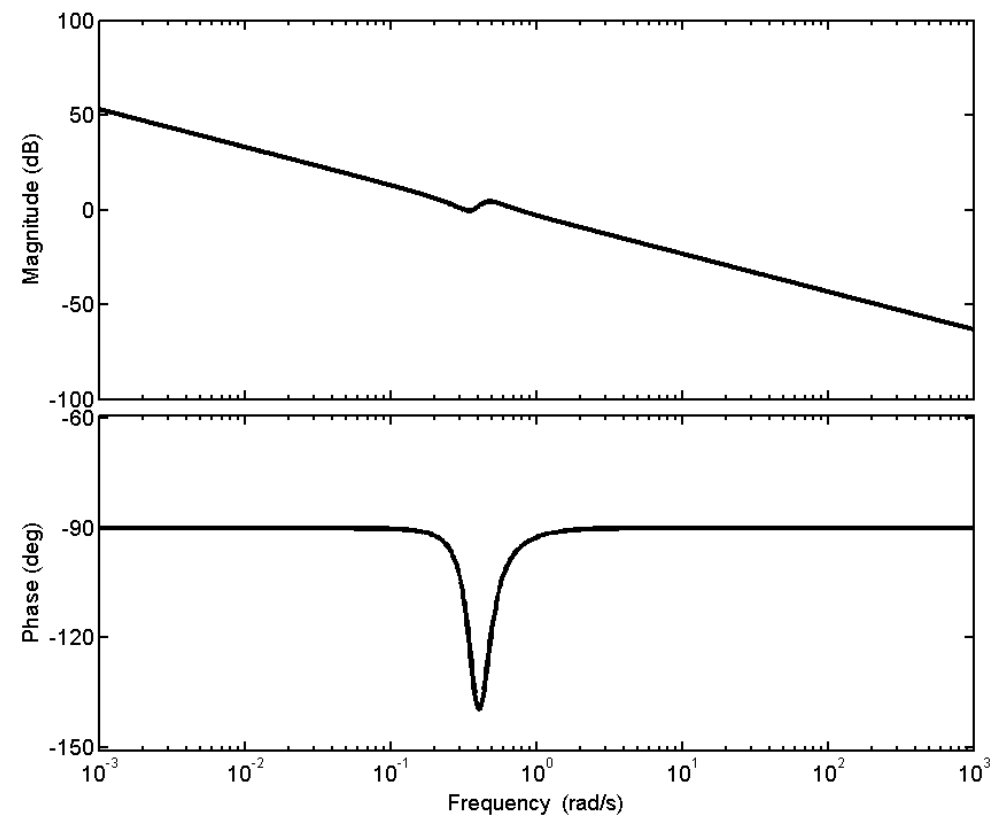

Figure 5.9: Bode diagram of the transfer function from differential thrust to yaw rate 
Table 5.1 shows that for the damaged aircraft, only two transfer functions have positive gain and phase margins, which are the transfer function from ailerons to roll angle $\left(\frac{\phi(s)}{\delta_{a}(s)}\right)$ and the transfer function from ailerons to roll rate $\left(\frac{p(s)}{\delta_{a}(s)}\right)$. The rest of the transfer functions have negative gain and/or phase margins, which signifies instability. This characteristic again demonstrates the tremendous effect of losing the vertical stabilizer.

Table 5.1: Open loop gain and phase margins of the damaged aircraft

\begin{tabular}{|c|c|c|}
\hline Transfer Functions & Gain Margin (dB) & Phase Margin (deg) \\
\hline$\frac{\phi(s)}{\delta_{a}(s)}$ & 8.54 & 91.35 \\
\hline$\frac{p(s)}{\delta_{a}(s)}$ & 41.75 & $\infty$ \\
\hline$\frac{\beta(s)}{\delta_{a}(s)}$ & Unstable & Unstable \\
\hline$\frac{r(s)}{\delta_{a}(s)}$ & Unstable & Unstable \\
\hline$\frac{\phi(s)}{\delta T(s)}$ & Unstable & Unstable \\
\hline$\frac{p(s)}{\delta T(s)}$ & Unstable & Unstable \\
\hline$\frac{\beta(s)}{\delta T(s)}$ & Unstable & Unstable \\
\hline$\frac{r(s)}{\delta T(s)}$ & Unstable & Unstable \\
\hline
\end{tabular}

Furthermore, Fig. 5.10 illustrates the comparison of the Bode diagrams for the nominal and damaged aircraft. For the nominal aircraft, the control inputs are ailerons $\left(\delta_{a}\right)$ and rudder $\left(\delta_{r}\right)$, whereas for the damaged aircraft, the control inputs are ailerons $\left(\delta_{a}\right)$ and differential thrust $(\delta T)$. From Fig. 5.10, it can be seen that for the aileron input, the Bode diagrams for the nominal and damaged aircraft match up fairly well at high frequency, but differ very much at low frequency. However, for the rudder/differential thrust input, the Bode diagrams for the nominal and damaged aircraft differ significantly, which represents the critical effect of losing the vertical stabilizer. 


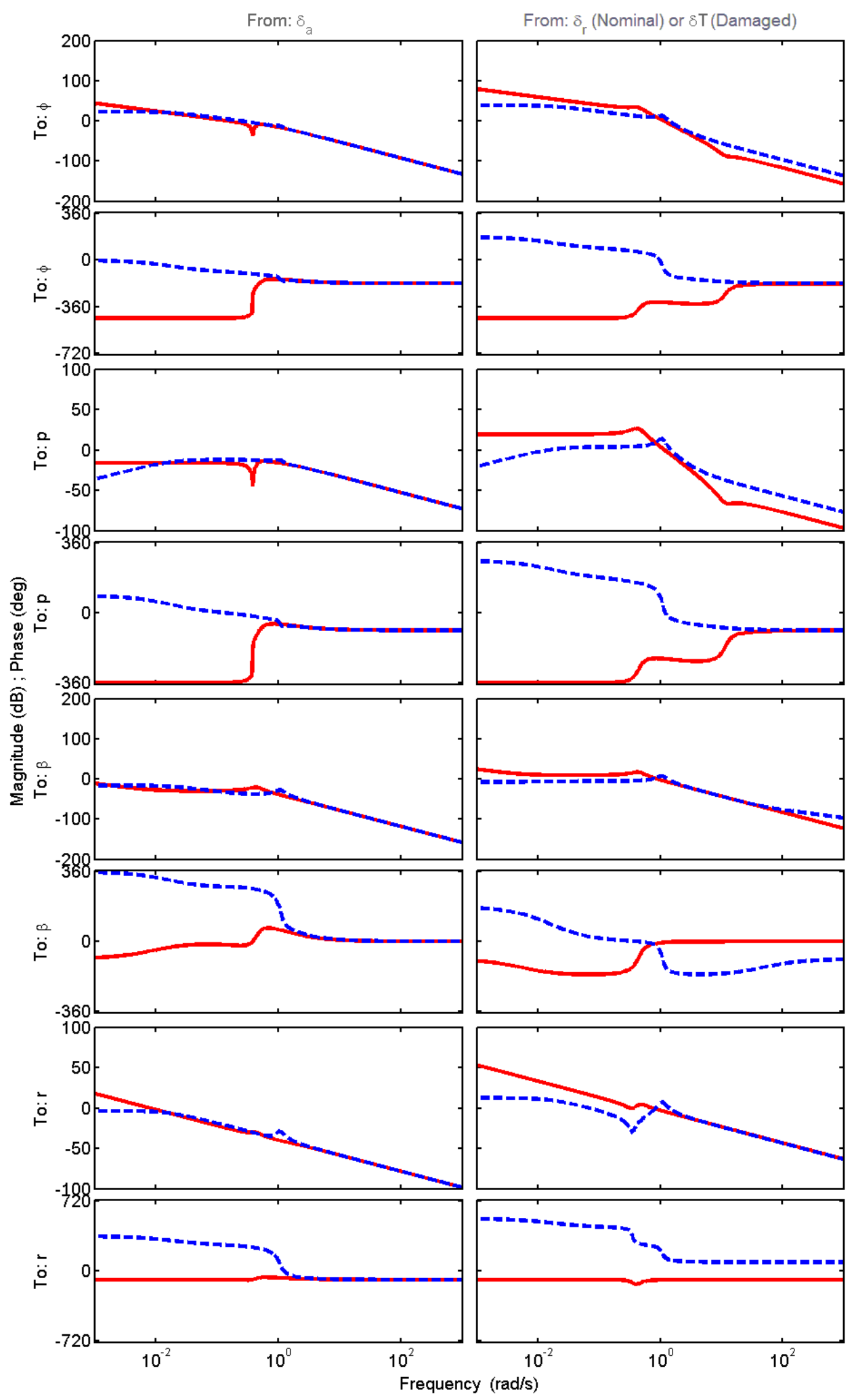

Figure 5.10: Bode diagrams of the nominal (blue) and damaged aircraft (red) 


\subsection{Robustness and Uncertainty Analysis of Frequency Response}

We investigate the damaged plant frequency response with associated $30 \%$ of full-block, additive uncertainty. We choose $30 \%$ to represent a real-life situation, where the designer is free to choose any bound and margin for uncertainty for analysis. The response is presented in Fig. 5.11, where it is obvious that even in the presence of uncertainty, high frequency dynamics of the aircraft remain (relatively) unchanged, except for the transfer function from ailerons to side-slip angle, while the low frequency content will remain within a ball of uncertainty. Moreover, for the differential thrust input, the plant remains fairly unchanged for side-slip angle and yaw rate in the presence of uncertainty, but for roll angle and roll rate, the plant experiences excitation at higher frequency range. However, for the aileron control input, lower frequencies experience more excitation than higher frequencies for roll angle and roll rate. Furthermore, for side-slip angle and yaw rate, the plant experiences excitation in both low and high frequencies.

\subsection{Chapter Summary}

In this chapter, we conducted the frequency domain analysis of the damaged aircraft and investigated its robustness by introducing 30\% full block, additive uncertainty to the damaged aircraft's plant matrix. In the next chapter, we are going to investigate the jet engine propulsion dynamics. 


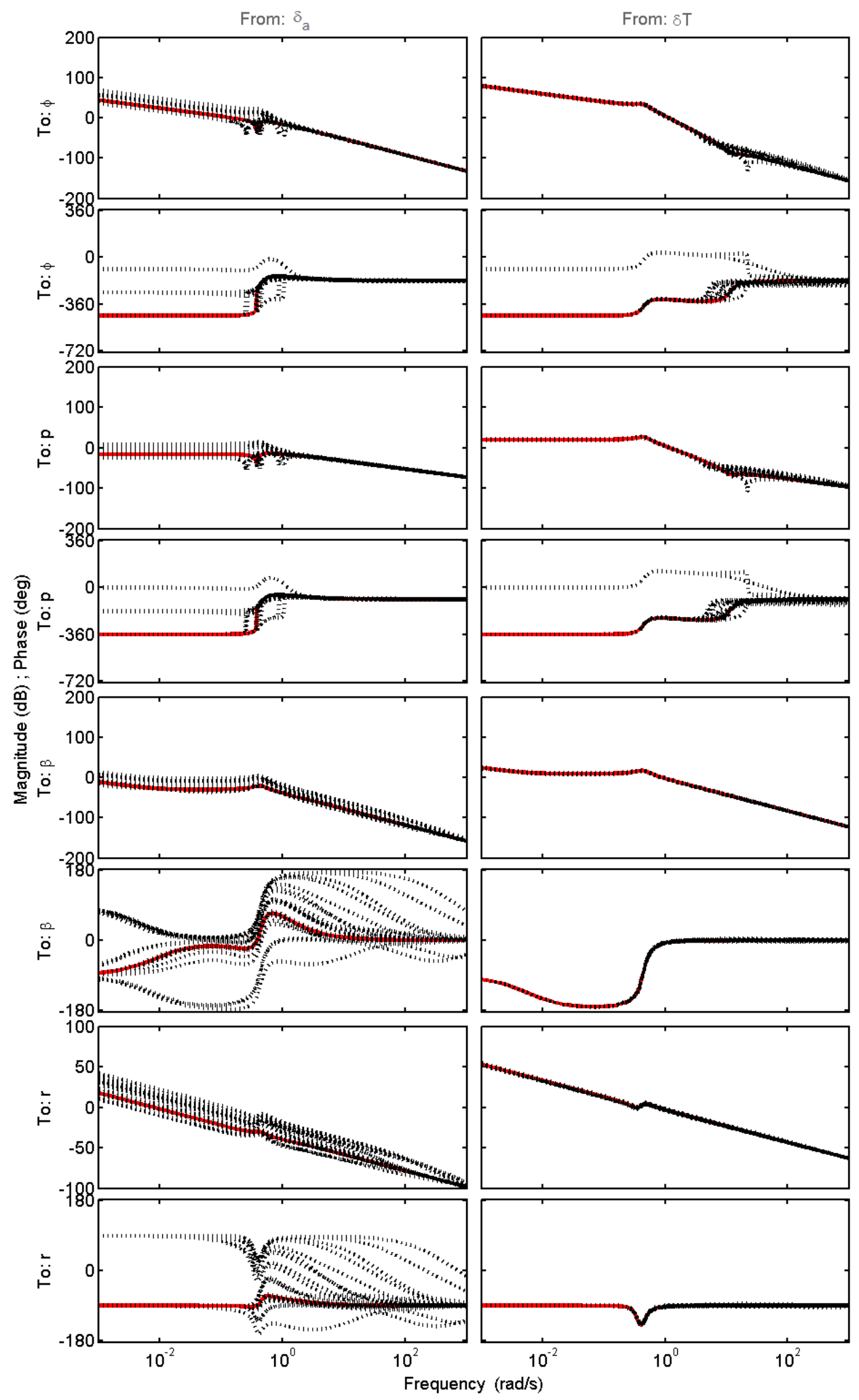

Figure 5.11: Bode diagrams of damaged (red) and uncertain plant (black) at $30 \%$ uncertainty 


\section{CHAPTER 6}

\section{PROPULSION DYNAMICS}

\subsection{Jet Engine Thrust Generation Dynamics}

With emerging advancements in manufacturing processes, structures, and materials, it is a well known fact that aircraft engines have become highly complex systems and include numerous nonlinear processes, which affect the overall performance (and stability) of the aircraft. From the force-balance point of view, this is usually due to the existing coupled and complex dynamics between engine components and their relationships in generating thrust. However, in order to utilize the differential thrust generated by the jet engines as a control input for lateral/directional stability, the dynamics of the engine need to be modeled to gain an insight into the response characteristics of the engines.

Engine responses, generally speaking, depend on the time constant and time delay characteristics. Time constant dictates how fast the thrust is generated by the engine, while time delay (which is inversely proportional to the initial thrust level) is due to the lag in engine fluid transport and the inertias of the mechanical systems such as rotors and turbo-machinery blades [6].

It is also suggested [6] that the non-linear engine dynamics model can be simplified as a time-delayed second-order linear model as

$$
\ddot{T}+2 \zeta \omega \dot{T}+\omega^{2} T=\omega^{2} T_{c}\left(t-t_{d}\right)
$$

where $\zeta$ and $\omega$ are the damping ratio and bandwidth frequency of the closed-loop 
engine dynamics, respectively; $t_{d}$ is the time delay factor; and $T_{c}$ is the thrust command prescribed by the engine throttle resolver angle.

With the time constant defined as the inverse of the bandwidth frequency $\left(\tau=\frac{1}{\omega}\right)$, and $\zeta$ chosen to be 1 representing a critically damped engine response (to be comparable to existing studies), the engine dynamics can be represented as

$$
\left[\begin{array}{c}
\dot{T} \\
\ddot{T}
\end{array}\right]=\left[\begin{array}{cc}
0 & 1 \\
\frac{-1}{\tau^{2}} & \frac{-2}{\tau}
\end{array}\right]\left[\begin{array}{c}
T \\
\dot{T}
\end{array}\right]+\left[\begin{array}{c}
0 \\
\frac{1}{\tau^{2}}
\end{array}\right] T_{c}\left(t-t_{d}\right)
$$

For this study, the Pratt and Whitney JT9D-7A engine is chosen for the application in the Boeing 747-100, where each engine produces a maximum thrust of 46,500 lbf [13]. The engine thrust response curve at Mach 0.65 and 20,000 feet is shown in Fig. 6.1.

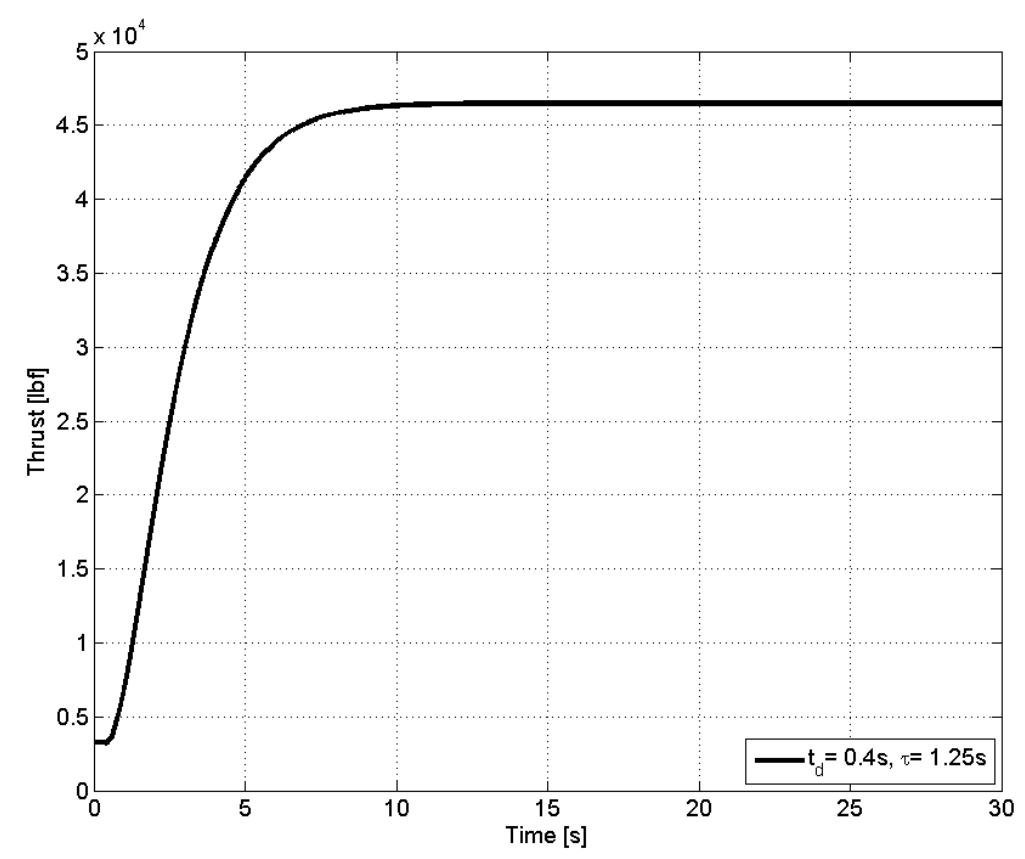

Figure 6.1: Engine thrust response at Mach 0.65 and 20,000 feet

Figure 6.1 provides a useful insight into how the time constant and time delay 
factors affect the generation of thrust for the JT9D-7A jet engine at Mach 0.65 and 20,000 feet. With the engine time constant of 1.25 seconds, and the time delay of 0.4 second [6], it takes approximately ten seconds for the engine to reach steady state and generate its maximum thrust capacity at 46,500 lbf from the trim thrust of 3221 lbf. The increase in thrust generation follows a relatively linear fashion with the engine response characteristic of approximately 12,726 lbf/s during the first two seconds, and then the thrust curve becomes nonlinear until it reaches its steady state at maximum thrust capacity after about ten seconds. This represents one major difference between the rudder and differential thrust as a control input. Due to the lag in engine fluid transport and turbo-machinery inertias, differential thrust (as a control input) cannot respond as instantaneously as the rudder, which has to be taken into account very seriously in control system design.

\subsection{Chapter Summary}

In this chapter, we investigated the thrust generation dynamics of the JT9D-7A jet engine and the effects of time constant and time delay on the engine thrust response characteristics. In the next chapter, we are developing a mechanism to utilize differential thrust as a control input to act as a "virtual rudder" to save the damaged aircraft. 


\section{CHAPTER 7}

\section{DIFFERENTIAL THRUST AS A CONTROL MECHANISM}

\subsection{Thrust Dynamics and Configuration}

In order to utilize differential thrust as a control input for the four-engined Boeing 747-100 aircraft, a differential thrust control module must be developed. Here, the differential thrust input is defined as the difference between the thrust generated by engine number 1 and engine number 4 while the amounts of thrust generated by engine number 2 and 3 are kept equal to each other as shown in Eqs. $(7.1-7.2)$.

$$
\begin{gathered}
\delta T=T_{1}-T_{4} \\
T_{2}=T_{3}
\end{gathered}
$$

This concept is illustrated in further details in Fig. 7.1. Engine number 1 and 4 are employed to generate the differential thrust due to the longer moment arm $\left(y_{e}\right)$, which makes the differential thrust more effective as a control for yawing moment. This brings into the picture the need of developing a logic that maps a rudder input to a differential thrust input, which is further explained in the following section.

\subsection{Rudder Input to Differential Thrust Input Mapping Logic}

When the vertical stabilizer of the aircraft is intact (i.e. with nominal plant dynamics), the pilot has the ailerons and rudder as major control inputs. However, 

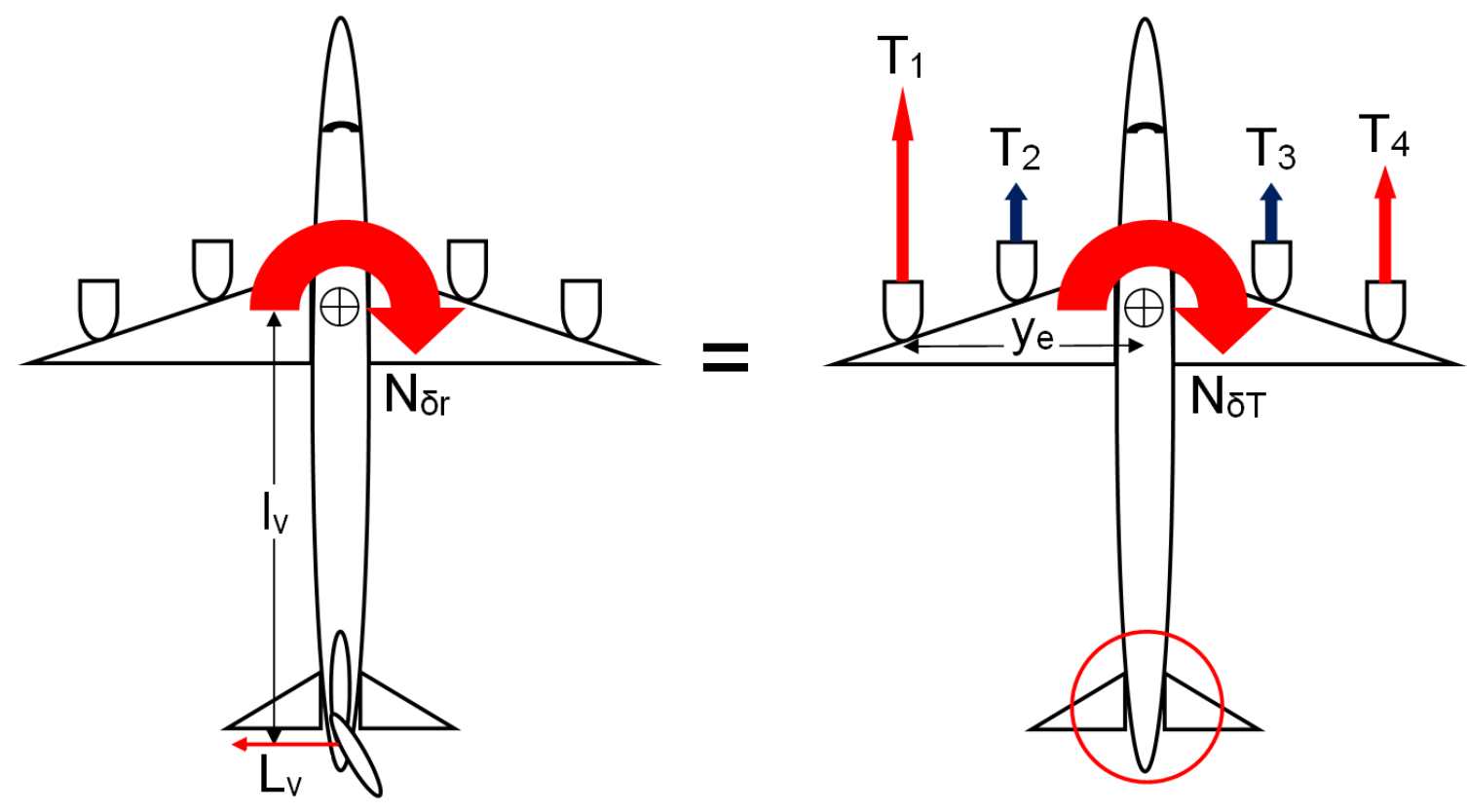

Figure 7.1: The free body diagram 
when the vertical stabilizer is damaged, most probably the pilot will keep on demanding the control effort from the rudder until it is clear that there is no response from the rudder. To eliminate this mishap, but to still be able to satisfy the rudder demand from the pilot, an aircraft control logic is introduced in Fig. 7.2.

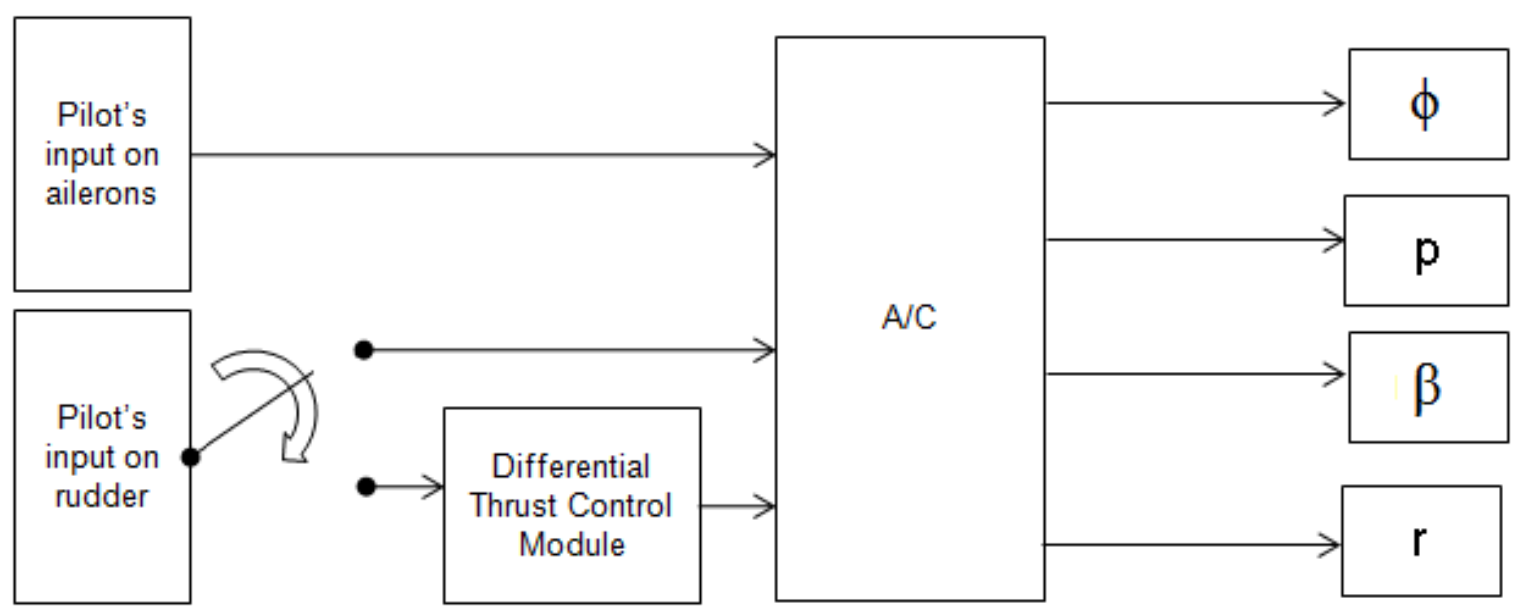

Figure 7.2: Aircraft control logic diagram

As seen in Fig. 7.2, the main goal is to map corresponding input/output dynamics from the rudder pedals to the aircraft responses, so that when the rudder is lost, the rudder input (from the pilot) will still be utilized but switched to the differential thrust input, which will act as the rudder input for lateral/directional controls. This logic constitutes one of the novel approaches introduced in this thesis.

This differential thrust control module is illustrated in Fig. 7.3. The differential thrust control module's function is to convert the rudder pedal input from the pilot to the differential thrust input. In order to achieve that, the rudder pedal input (in radians) is converted to the differential thrust input (in pounds-force) which is then provided into the engine dynamics, as discussed previously in Chapter 6 . With this modification, the engine dynamics will dictate how differential thrust is generated, which is then provided as a "virtual rudder" 
input into the aircraft dynamics. The radian to pound-force conversion is derived in the next section.

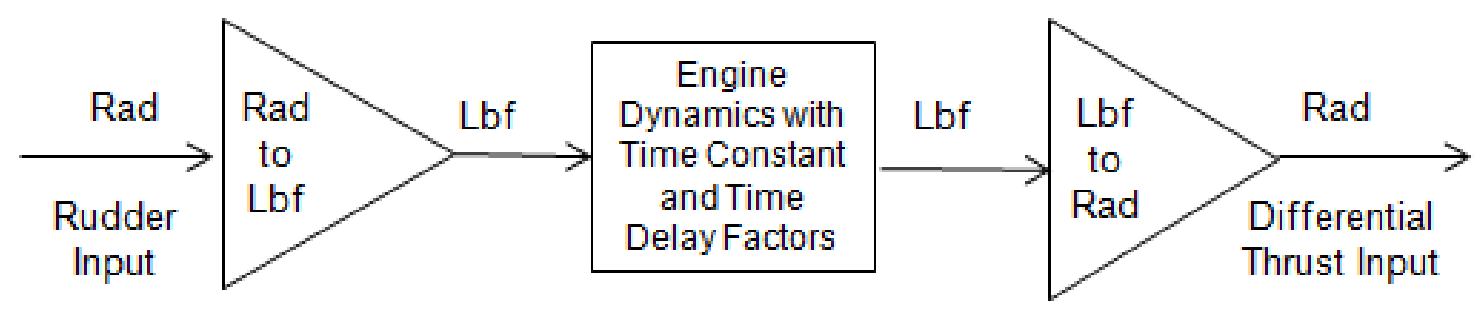

Figure 7.3: Differential thrust control module

\subsection{Radian to Pound-Force Conversion Factor}

Using Fig. 7.1 and with the steady, level flight assumption at the altitude of 20,000 feet, the following relationship can be obtained:

$$
\begin{gathered}
N_{\delta_{r}}=N_{\delta T} \\
q S b C_{N \delta_{r}} \delta_{r}=(\delta T) y_{e}
\end{gathered}
$$

which means the yawing moment by deflecting the rudder and by using differential thrust have to be the same. Therefore, the relationship between the differential thrust control input $(\delta T)$ and the rudder control input $\left(\delta_{r}\right)$ can be obtained as

$$
\delta T=\left(\frac{q S b C_{N \delta_{r}}}{y_{e}}\right) \delta_{r}
$$

Based on the flight conditions at Mach 0.65 and 20,000 feet, and the data for the Boeing 747-100 summarized in Table 3.1 and Table 3.2, the conversion factor for the rudder control input to the differential thrust input is calculated to be

$$
\frac{\delta T}{\delta_{r}}=-4.43 * 10^{5} \frac{\mathrm{lbf}}{\mathrm{rad}}
$$


Due to the sign convention of rudder deflection and the free body diagram in Fig. 7.1, $\delta_{r}$ here is negative. Therefore, for the Boeing 747-100, in this study, the conversion factor for the mapping of a rudder input to a differential thrust input is found to be

$$
\frac{\delta T}{\delta_{r}}=4.43 * 10^{5} \frac{l b f}{r a d}
$$

\subsection{Commanded vs. Available Differential Thrust}

At this point, the worst case scenario is considered, and it is assumed that the aircraft has lost its vertical stabilizer so that the rudder input is converted to the differential thrust input according to the logic discussed previously in this chapter.

Unlike the rudder, due to delayed engine dynamics with time constant, there is a major difference in the commanded differential thrust and the available differential thrust as shown in Fig. 7.4.

It can be seen from Fig. 7.4 that compared to the commanded differential thrust, the available differential thrust is equal in amount but longer in time delivery. For a one degree step input on the rudder, the corresponding equivalent commanded and available differential thrust amounts are $7737 \mathrm{lbf}$, which is deliverable in ten seconds. Unlike the instantaneous control of the rudder input, there is a lag associated with the use of differential thrust as a control input. This is due to the lag in engine fluid transport and the inertias of the mechanical systems such as rotors and turbo-machinery blades [6]. This is a major design consideration and will be taken into account during the control system design phases. 


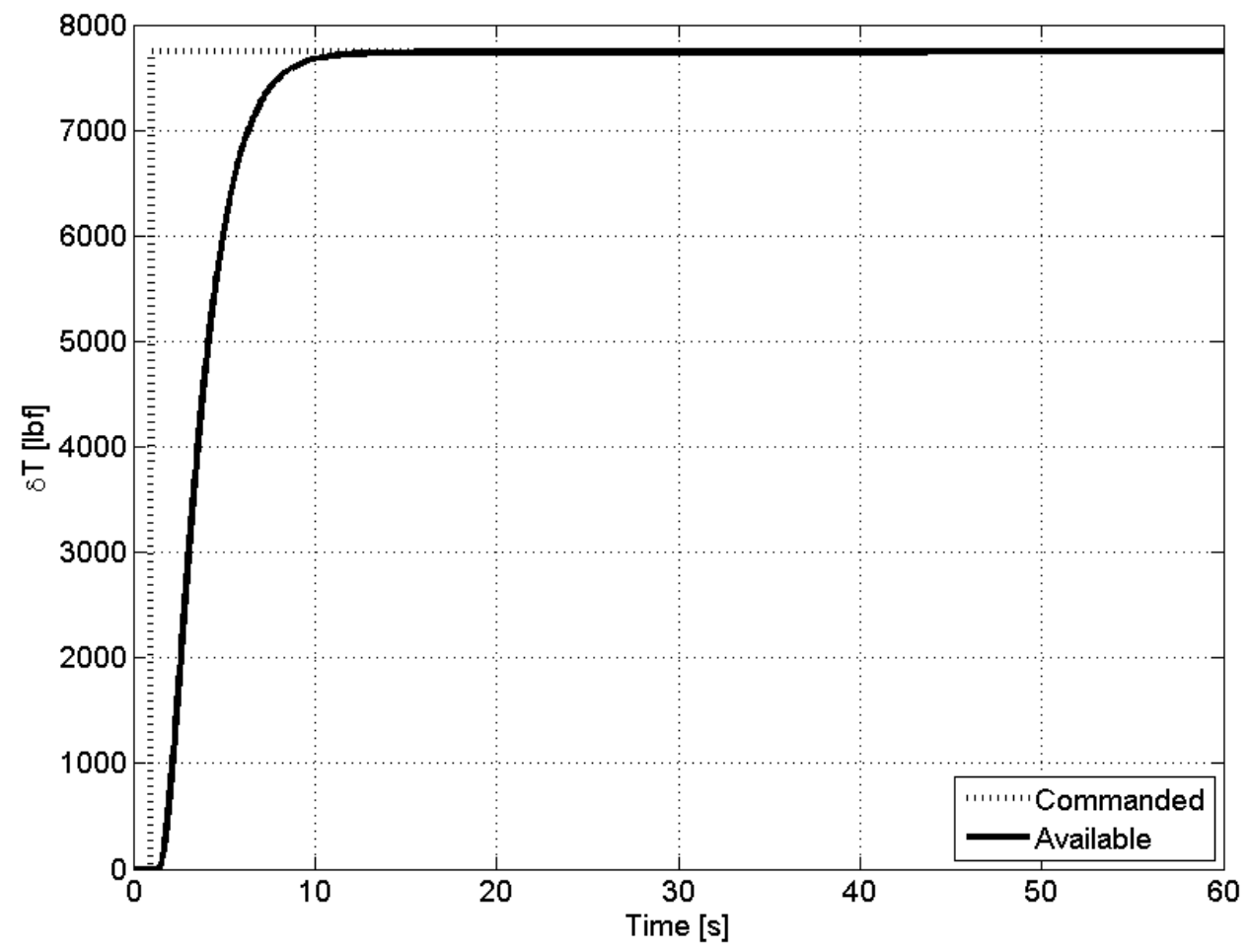

Figure 7.4: Commanded vs. available differential thrust 


\subsection{Chapter Summary}

In this chapter, we developed the mapping logic between the rudder input and differential thrust input. We also derived the radian to pound-force conversion factor, which is of extreme importance to the utilization of differential thrust as a control mechanism to save the damaged aircraft. We also conducted the comparison between commanded and available differential thrust. In the next chapter, we are investigating the open loop responses of the damaged aircraft. 


\section{CHAPTER 8}

\section{OPEN LOOP SYSTEM RESPONSE ANALYSIS}

\subsection{Open Loop Responses of the Damaged Aircraft}

The open loop response characteristics of the aircraft with a damaged vertical stabilizer to one degree step inputs from the ailerons and differential thrust are presented in Fig. 8.1, where it can be clearly seen that when the aircraft is majorly damaged and the vertical stabilizer is lost, the aircraft responses to the pilot's inputs are completely unstable in all four states (as it was also obvious from the pole locations). This means the pilot will not have much chance to stabilize the aircraft in time, which calls for a novel approach to save the damaged aircraft. This is another point where the second novel contribution of the thesis is introduced: automatic control strategies to stabilize the aircraft, which allows safe (i.e. intact) landing of the aircraft.

\subsection{Chapter Summary}

In this chapter, we conducted the open loop system response analysis of the damaged aircraft. It is concluded that the open loop responses of the damaged aircraft are very unstable, and the pilot will not have much chance to stabilize the aircraft. This calls for the design and implementation of automatic control systems to save the damaged aircraft. However, before a control system can be designed to stabilize and save the damaged aircraft, the controllablity of the damaged aircraft has to be investigated in the next chapter. 

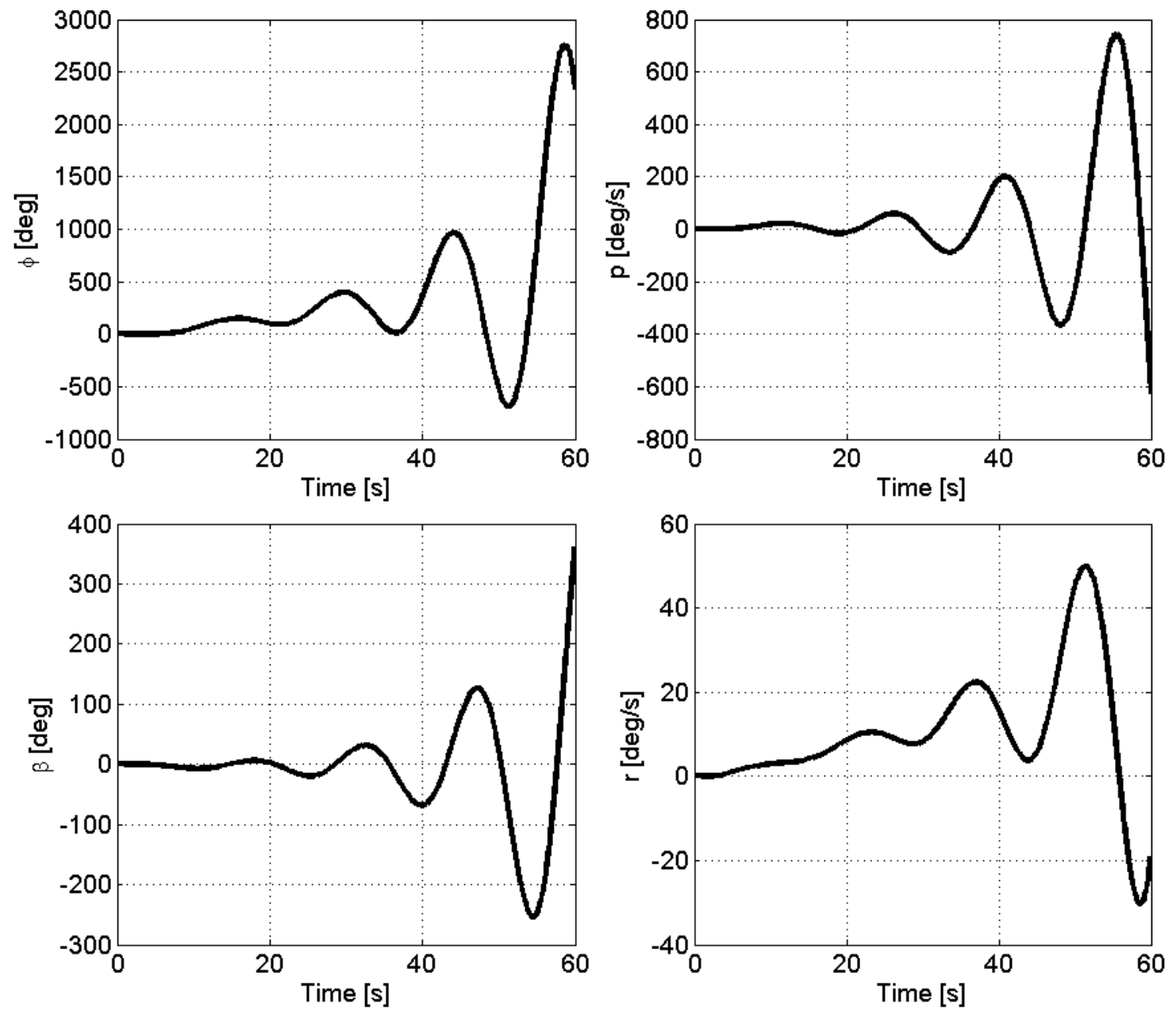

Figure 8.1: Open loop responses of the damaged aircraft 


\section{CHAPTER 9}

\section{CONTROLLABILITY OF THE DAMAGED AIRCRAFT}

\subsection{Controllability of the Damaged Aircraft}

Before a controller to stabilize the damaged aircraft can be designed, the controllability of the damaged aircraft system must be investigated.

Controllability is defined as the ability to drive an initial condition $x_{o}(t)$ to a final condition $x_{f}(t)$ at a given finite amount of time $\left[t_{o}, t_{f}\right]$. The controllability matrix of a system is defined as

$$
C o=\left[\begin{array}{llll}
B & A B & A^{2} B \cdots & A^{n-1} B
\end{array}\right]
$$

In this study, $A=A_{d}, B=B_{d}$, and $n=4$ (because there are four states: $\phi, p, \beta$, and $r$, which represent the roll angle, roll rate, side-slip angle, and yaw rate, respectively). Therefore,

$$
C o=\left[\begin{array}{cccc}
B_{d} & A_{d} B_{d} & A_{d}^{2} B_{d} & A_{d}^{3} B_{d}
\end{array}\right]
$$

In order for the system to be controllable, $\operatorname{Rank}(C o)=n=4$. For the damaged aircraft, the controllability matrix is obtained as

$$
C o=\left[\begin{array}{cccccccc}
0 & 0 & 0.2249 & 0.0142 & -0.1915 & 0.0562 & 0.1960 & 1.8297 \\
0.2249 & 0.0142 & -0.1915 & 0.0562 & 0.1960 & 1.8297 & -0.2126 & -1.5702 \\
0 & 0 & -0.0118 & -0.6784 & 0.0163 & 0.0010 & -0.0139 & 0.0041 \\
0.0118 & 0.6784 & -0.0056 & -0.0004 & 0.0047 & -0.0014 & -0.0049 & -0.0453
\end{array}\right]
$$


$\operatorname{Rank}(\mathrm{Co})=4$, so the controllability matrix has full rank, which leads to a fully controllable system. This also means that all four lateral/directional states are linearly independent, and we are able to manipulate each state separately by the control inputs. As a confirmation, it is worth checking if there is any uncontrollable state.

$$
U n C o=\operatorname{length}(A)-\operatorname{Rank}(C o)=4-4=0
$$

Therefore, it can be confirmed that there is no uncontrollable state.

\subsection{Chapter Summary}

In this chapter, we investigated the controllablity of the damaged aircraft and concluded that the damaged aircraft plant is controllable. Therefore, in the next chapter, we are conducting the linear quadratic regulator control system design to stabilize and save the damaged aircraft. 


\section{CHAPTER 10}

\section{LINEAR QUADRATIC REGULATOR CONTROL SYSTEM DESIGN}

\subsection{Background Theory}

Optimal control aims for the best results with maximum benefits and minimum control efforts within a given set of constraints. An optimal controller is designed to minimize a performance index (PI), which constitutes a desired objective function and is defined as the ultimate collection of all valuable metrics that are of interest to the designer. In the following, we present the linear quadratic regulator (LQR).

Taken from [17], consider the optimal regulator problem:

Given the system equation:

$$
\dot{x}=A x+B u
$$

Determine the gain matrix $K$ of the optimal control vector:

$$
u=-K x
$$

to minimize the performance index:

$$
J=\int_{0}^{\infty}\left(x^{T} Q x+u^{T} R u\right) d t
$$

where $\mathrm{Q}$ and $\mathrm{R}$ are real and positive definite symmetric matrices. It is worth noting that Eq. (10.3) represents the performance index (PI), in which $x^{T} Q x$ represents the transient energy cost and $u^{T} R u$ represents the control energy cost. Therefore, $\mathrm{Q}$ becomes the state weighting matrix, and $\mathrm{R}$ becomes the control cost matrix. 
By applying the optimal linear quadratic regulator, we investigate the full state feedback structure.

Substituting Eq. (10.2) into Eq. (10.1), we have

$$
\dot{x}=A x+B K x=(A-B K) x
$$

Assuming that $(A-B K)$ is Hurwitz, then $(A-B K)$ becomes strictly negative definite (which means all the poles of $(A-B K)$ are on the left half plane (LHP)).

Substituting Eq. (10.2) into Eq. (10.3), we obtain

$$
J=\int_{0}^{\infty}\left(x^{T} Q x+x^{T} K^{T} R K x\right) d t=\int_{0}^{\infty} x^{T}\left(Q+K^{T} R K\right) x d t
$$

Now let us set

$$
x^{T}\left(Q+K^{T} R K\right) x=-\frac{d}{d t}\left(x^{T} P x\right)
$$

where $P$ is a real positive symmetric matrix. We next have

$$
x^{T}\left(Q+K^{T} R K\right) x=-\dot{x}^{T} P x-x^{T} P \dot{x}=-x^{T}\left[(A-B K)^{T} P+P(A-B K)\right] x
$$

Comparing both sides of Eq. (10.7), we obtain

$$
-\left(Q+K^{T} R K\right)=(A-B K)^{T} P+P(A-B K)
$$

Due to $R$ being a real positive symmetric matrix, we can rewrite $R$ as

$$
R=T^{T} T
$$

where $T$ is a non-singular matrix. Next, Eq. (10.8) can be written as 


$$
\begin{array}{r}
\left(A^{T}-K^{T} B^{T}\right) P+P(A-B K)+Q+K^{T} T^{T} T K=0 \\
\rightarrow A^{T} P+P A+\left[T K-\left(T^{T}\right)^{-1} B^{T} P\right]^{T}\left[T K-\left(T^{T}\right)^{-1} B^{T} P\right]-P B R^{-1} B^{T} P+Q=0
\end{array}
$$

We can see that the minimization of $J$ with respect to $K$ requires the minimization (with respect to $K$ ) of $x^{T}\left[T K-\left(T^{T}\right)^{-1} B^{T} P\right]^{T}\left[T K-\left(T^{T}\right)^{-1} B^{T} P\right] x$. Since $x^{T}\left[T K-\left(T^{T}\right)^{-1} B^{T} P\right]^{T}\left[T K-\left(T^{T}\right)^{-1} B^{T} P\right] x$ is non-negative, the minimum occurs when $x^{T}\left[T K-\left(T^{T}\right)^{-1} B^{T} P\right]^{T}\left[T K-\left(T^{T}\right)^{-1} B^{T} P\right] x=0$. Therefore,

$$
T K=\left(T^{T}\right)^{-1} B^{T} P \rightarrow K=T^{-1}\left(T^{T}\right)^{-1} B^{T} P=R^{-1} B^{T} P
$$

Equation (10.11) gives us the formulation for finding the optimal $K$, and this matrix $K$ must satisfy Eq. (10.8). Hence,

$$
A^{T} P+P A-P B R^{-1} B^{T} P+Q=0
$$

Equation (10.12) is called the reduced Riccati equation. Therefore, in order to design the linear quadratic regulator (LQR), one must follow these steps:

(1) Solve Eq. (10.12), the reduced Riccati equation, for the matrix $P$. It is worth noting that if matrix $P$ is a positive definite matrix, the system is stable (matrix $(A-B K)$ is stable).

(2) Substitute matrix $P$ back into Eq. (10.11) to get the optimal feedback matrix gain. 


\subsection{LQR Control System Design}

The damaged aircraft plant is stabilized by a full-state feedback linear quadratic regulator (LQR) controller as shown in Fig. 10.1.

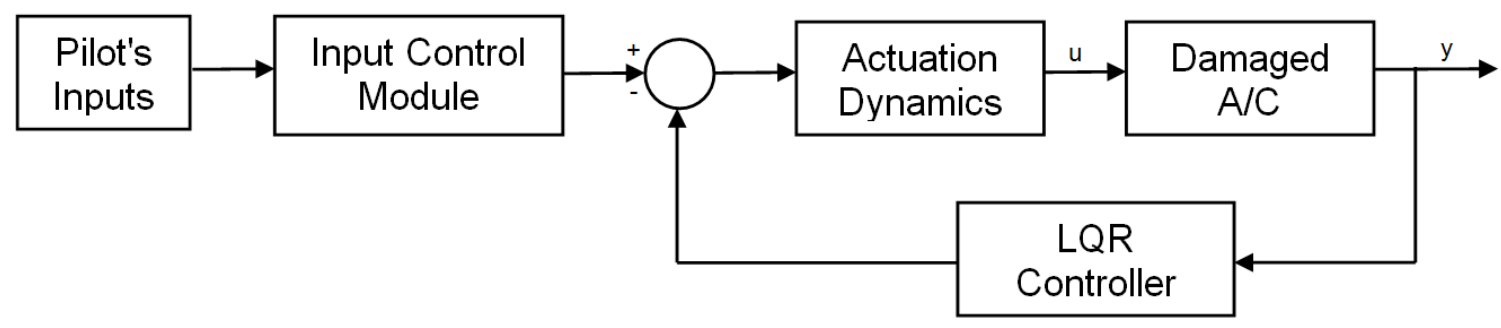

Figure 10.1: Block diagram for full-state feedback LQR control system design

As seen in Fig. 10.1, the pilot's inputs, which are one degree step inputs for both ailerons and rudder, will go through the Input Control Module, where the aileron control signal is routed through the saturation values of \pm 26 degrees [18] and the rudder control input is routed through the Differential Thrust Control Module, where the rudder input is converted to differential thrust input as discussed previously in Chapter 7. It is also worth noting that there are also a differential thrust saturation value of 43,729 lbf and a thrust generation rate limiter of 12,726 lbf/s imposed on the differential thrust control as discussed in Chapter 7. This is to make sure the control inputs are within the limits of both the ailerons and differential thrust. Furthermore, in order for the control efforts to be feasible in a real-life scenario, the control effort signals are also routed through the Actuation Dynamics, where the same saturation values and rate limiters are imposed on the ailerons and differential thrust as discussed previously.

After an iterative process, the state weighting matrix, Q, and the control cost matrix, $\mathrm{R}$, are chosen as 


$$
\begin{gathered}
Q=10^{5}\left[\begin{array}{cccc}
1 & 0 & 0 & 0 \\
0 & 2 & 0 & 0 \\
0 & 0 & 0.1 & 0 \\
0 & 0 & 0 & 1
\end{array}\right] \\
R=10^{3}\left[\begin{array}{ll}
1 & 0 \\
0 & 1
\end{array}\right]
\end{gathered}
$$

The feedback matrix $K$ is then obtained as

$$
K_{L Q R}=\left[\begin{array}{cccc}
9.6697 & 13.2854 & -9.1487 & 0.8729 \\
1.9631 & 2.8644 & -12.1067 & 11.5702
\end{array}\right]
$$

The model plant matrix, $A_{m}=A_{d}-B_{d} K_{L Q R}$, is then calculated to be

$$
A_{m}=\left[\begin{array}{cccc}
0 & 1 & 0 & 0 \\
-2.2026 & -3.8851 & -0.5390 & -0.2595 \\
0.0478 & 0 & 0 & -1 \\
-1.4455 & -2.1243 & 8.3210 & -7.8597
\end{array}\right]
$$

Next, let's investigate the closed loop responses of the aircraft with an LQR controller as shown in Fig. 10.2 and Fig. 10.3, and the closed loop control efforts as depicted in Fig. 10.4.

Compared to the unstable open loop responses of the damaged aircraft in Fig. 8.1 in Chapter 8, the closed loop responses of the damaged aircraft are stable in all four states: roll angle $(\phi)$, roll rate $(p)$, side-slip angle $(\beta)$, and yaw rate $(r)$. From Fig. 10.2, we can clearly see that all four states reach steady state only after 15 seconds. It is also worth noticing that with the help of differential thrust acting as a "virtual rudder" input during the coordinated turn maneuver, the aircraft was able 

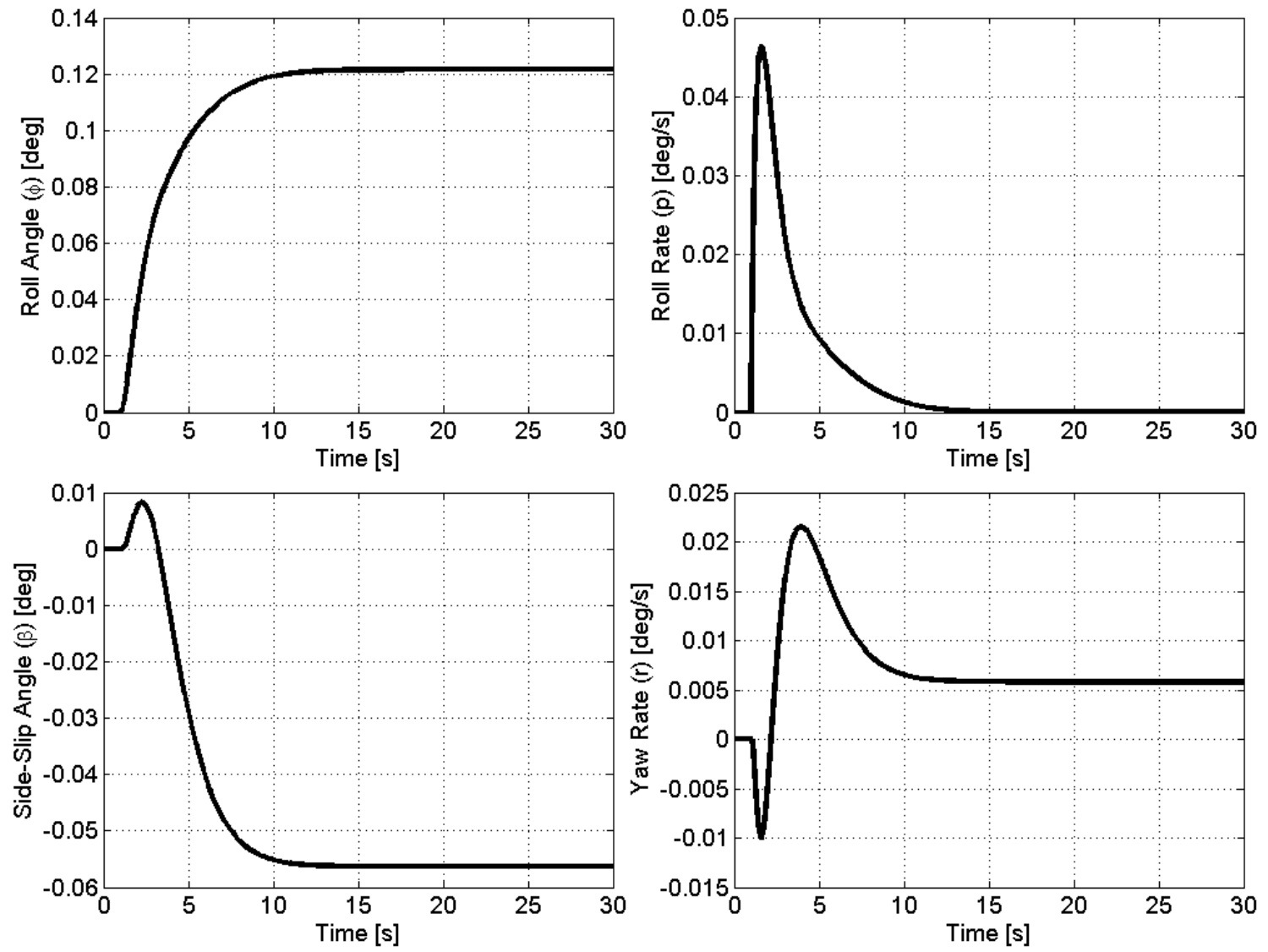

Figure 10.2: Closed loop responses of the damaged aircraft with an LQR controller 


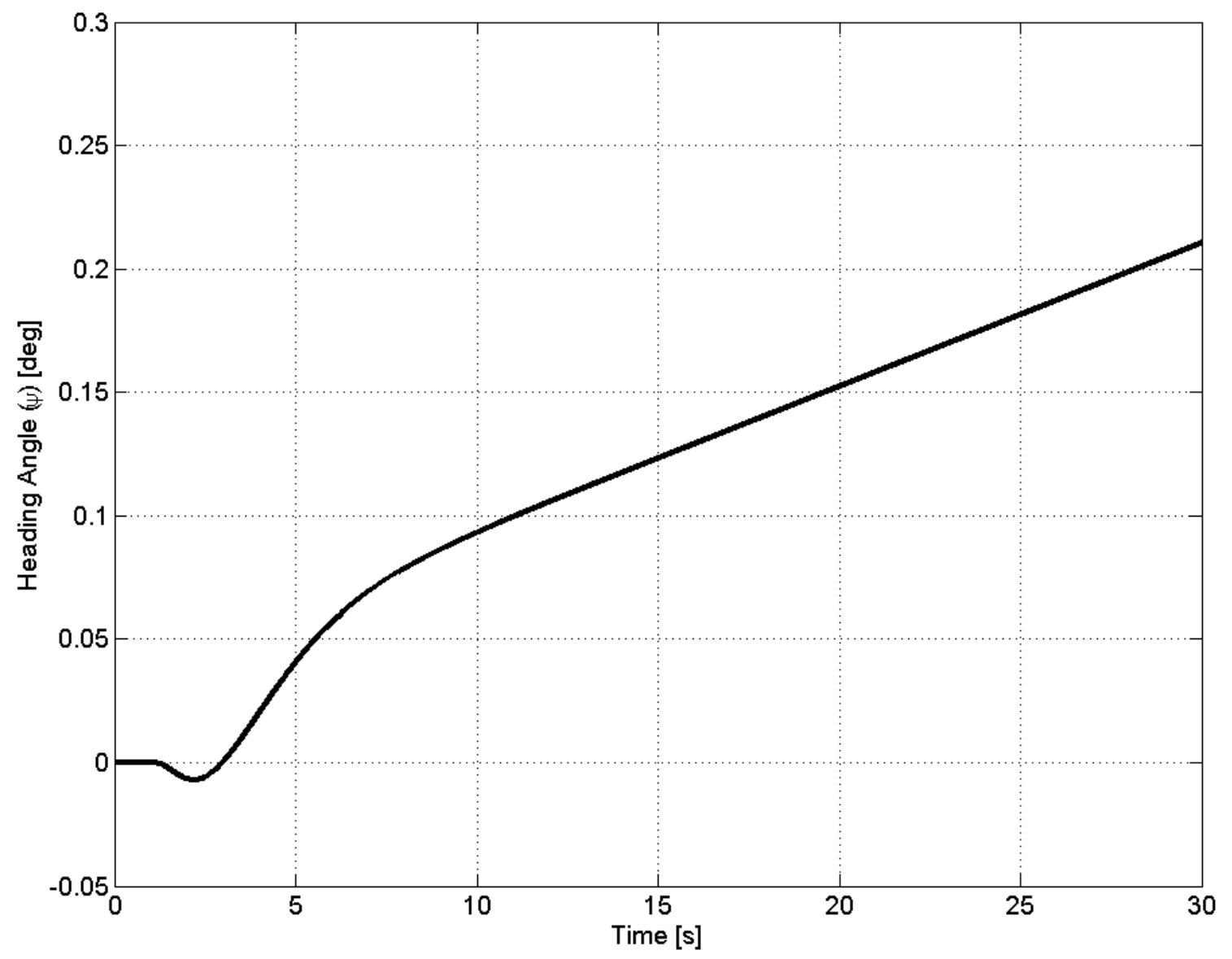

Figure 10.3: Closed loop heading angle response with an LQR controller 

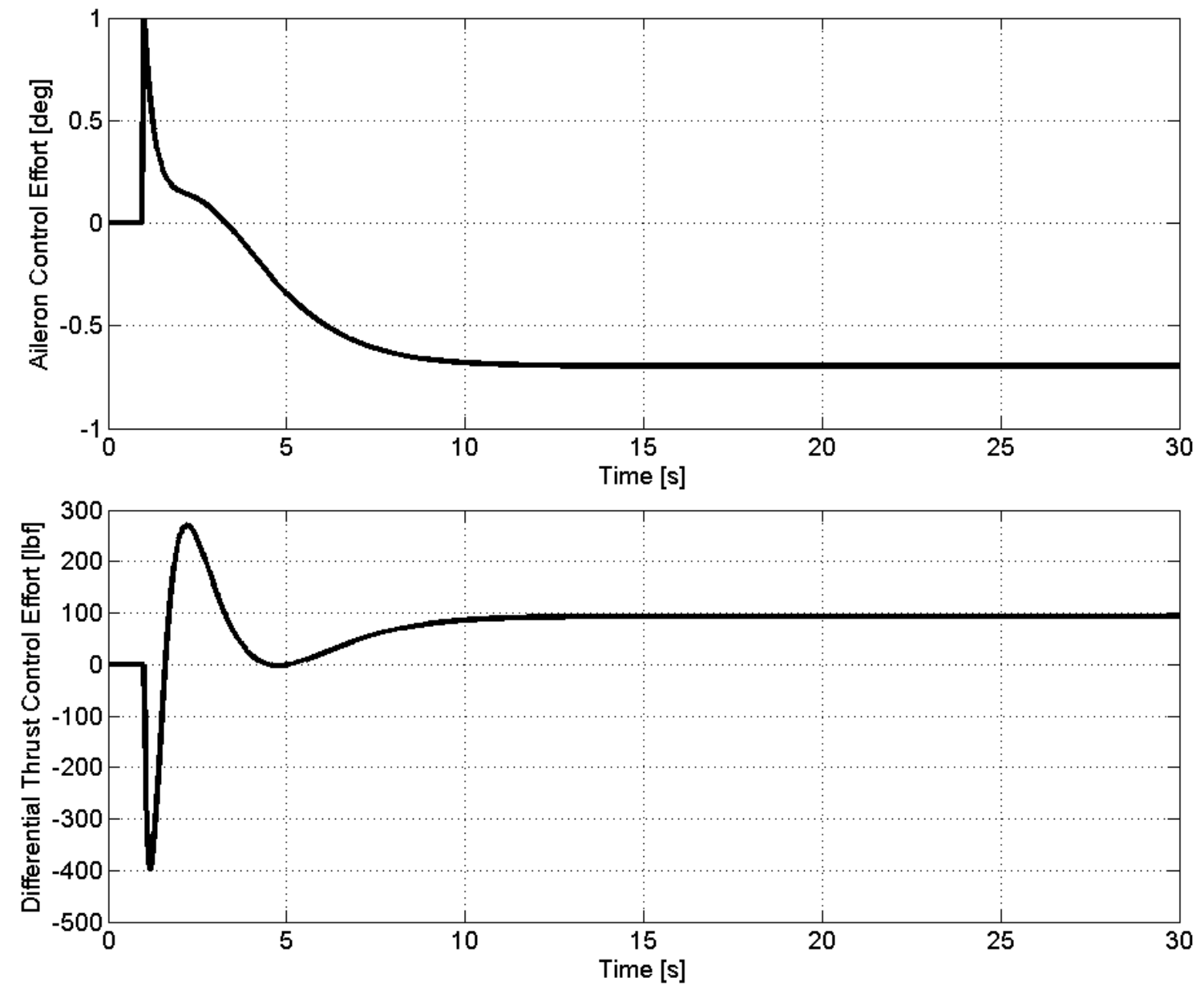

Figure 10.4: Closed loop control efforts with an LQR controller 
to achieve very close to zero side-slip angle $(\beta=-0.057 \mathrm{deg})$. Furthermore, as seen in Fig. 10.3, the heading angle gain is very linear after 15 seconds, and for one degree step inputs, the heading angle gain is about 0.22 degree after 30 seconds. In addition, the control efforts for ailerons and differential thrust are also feasible. From Fig. 10.4, we can see that aileron control effort demands the maximum deflection of 1 degree and settles at around -0.7 degree while differential thrust control effort demands a maximum of -400 lbf (negative differential thrust means $T 4>T 1$ ) and settles at approximately $100 \mathrm{lbf}$, which is within the thrust capability of the JT9D-7A engine.

\subsection{Robustness and Sensitivity Analysis of LQR Control System}

The robustness and sensitivity of the LQR control system are investigated by the introduction of band-limited white noise and additive uncertainty parameters associated with the state matrix of the damaged aircraft to investigate the performance of the LQR control system in the presence of noise and uncertainty.

\subsubsection{Noise Sensitivity Analysis of LQR Control System}

The noise sensitivity analysis of the LQR control system design is conducted by introducing band-limited white noise to test the performance of the LQR control system in the presence of noise. Fig. 10.5 shows the logic behind the noise sensitivity analysis of the LQR control system. 


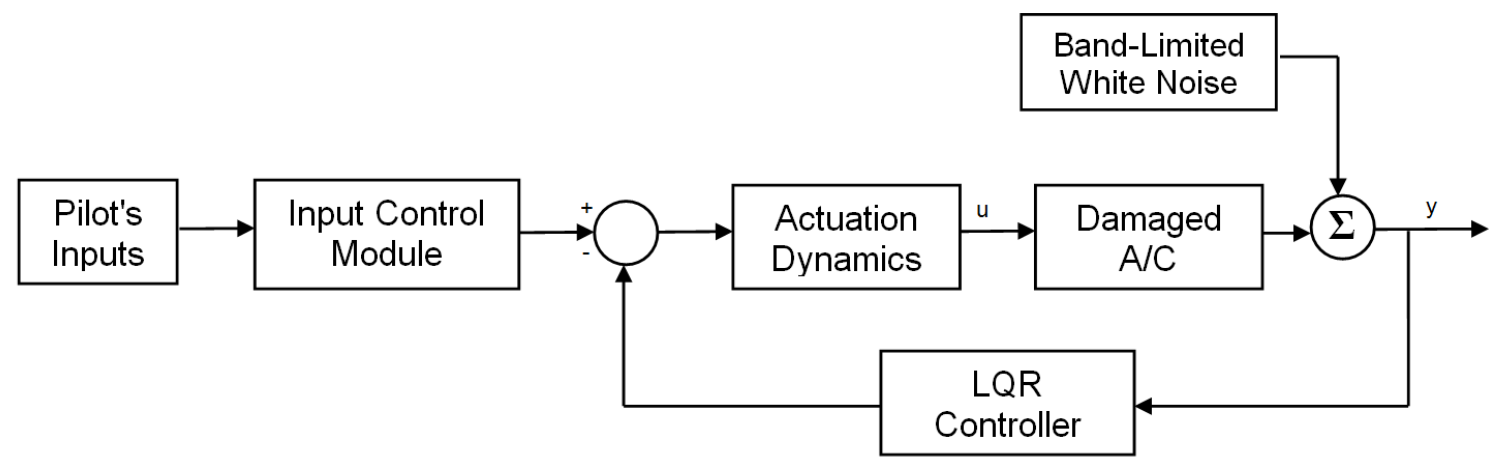

Figure 10.5: Block diagram for full-state feedback LQR control system design in the presence of noise

The noise power is set at $10^{-8}$ with sampling time of 0.1 second. The band-limited white noise for the LQR controller is depicted in Fig. 10.6, and its periodogram using Fast Fourier Transform (FFT) is shown in Fig. 10.7.

Additionally, the closed loop responses of the damaged aircraft with an LQR controller in the presence of band-limited white noise are shown in Fig. 10.8 and Fig. 10.9.

From Fig. 10.8, it is obvious that the LQR control system design is able to stabilize the damaged aircraft within only 15 seconds in the presence of noise. We can also see that all four state outputs are affected by the band-limited white noise, but the variations are well within the steady state bounds.

As for the heading angle response $(\psi)$ shown in Fig. 10.9, the effect of noise is attenuated by the integral action (because the heading angle $(\psi)$ is the integral of the yaw rate $(r)$ ), and the heading angle gain stays the same as when there is no noise: for one degree step inputs, the heading angle gain is about 0.22 degree within 30 seconds.

Furthermore, it is also worth investigating the control efforts required in the presence of noise as shown in Fig. 10.10. 


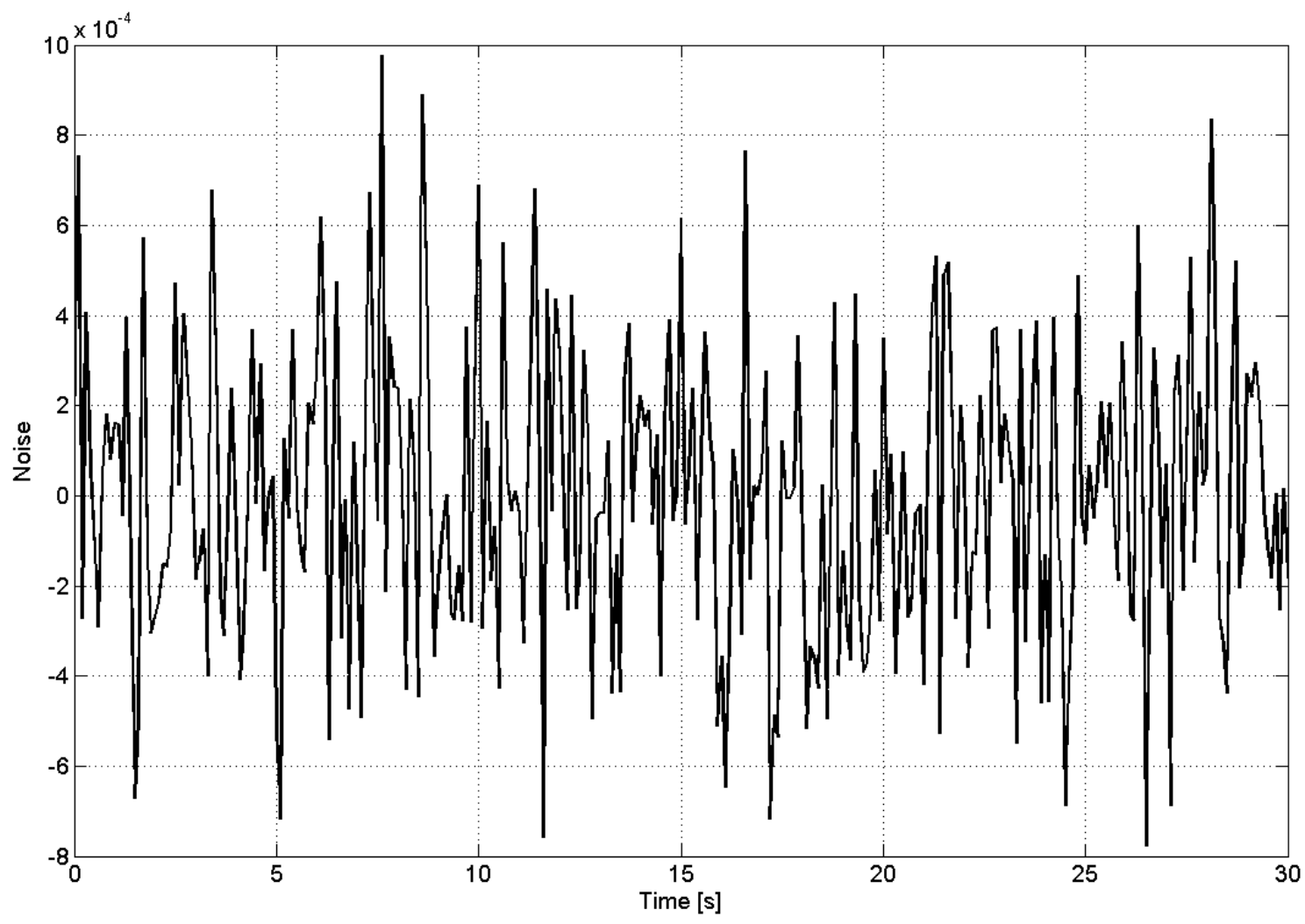

Figure 10.6: Band-limited white noise 


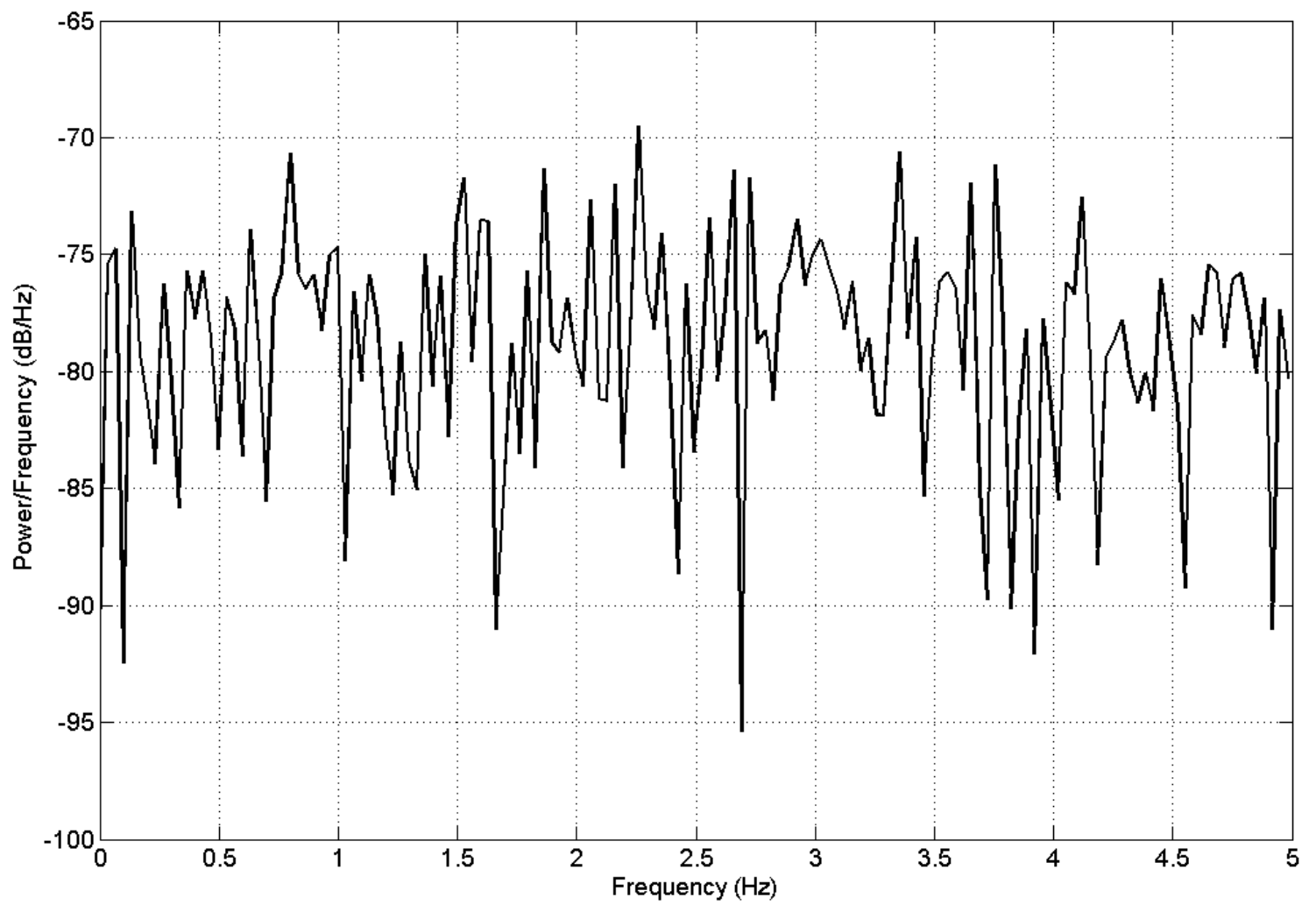

Figure 10.7: Periodogram using Fast Fourier Transform 

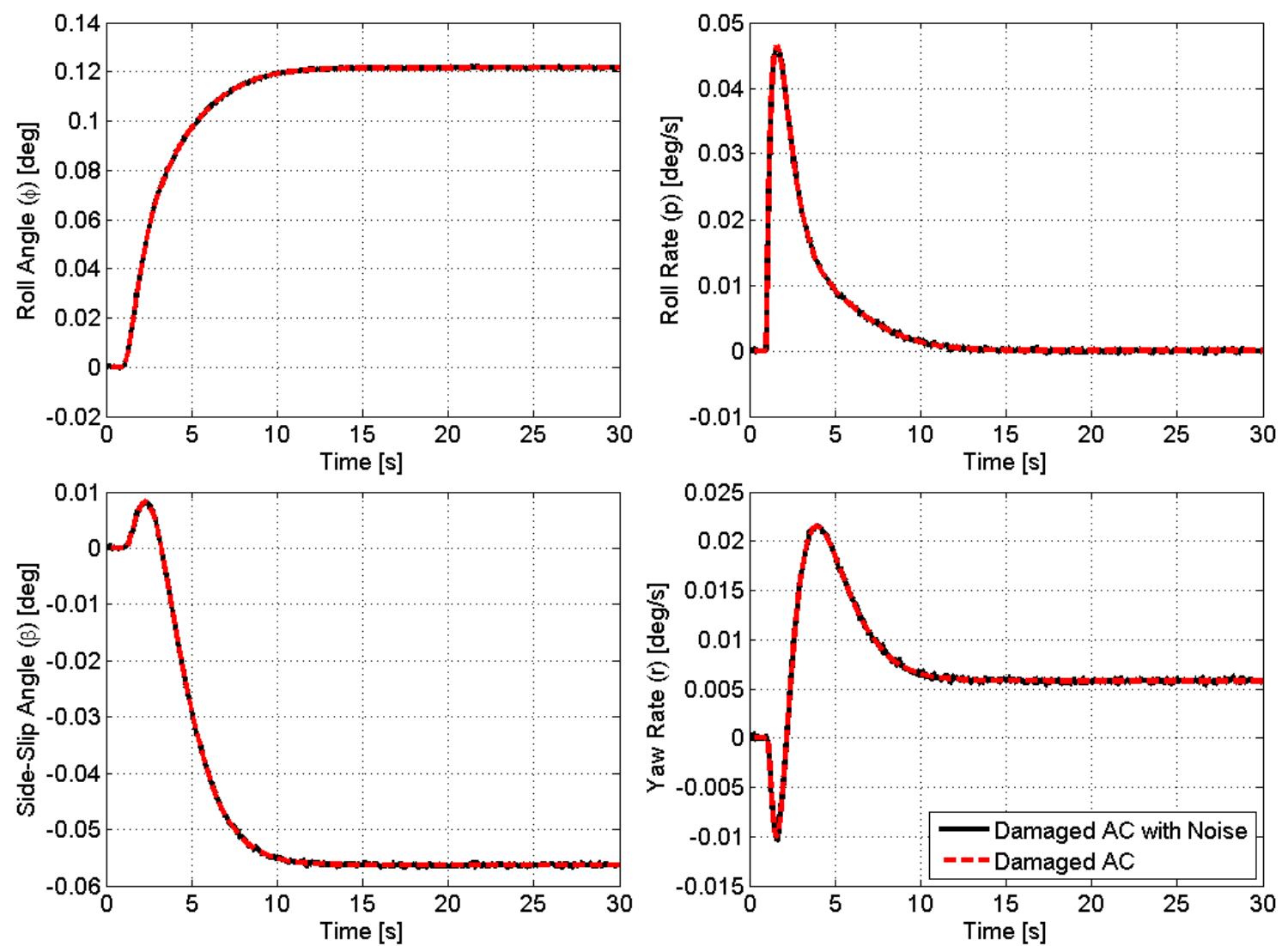

Figure 10.8: Closed loop responses of the damaged aircraft with an LQR controller in the presence of noise 


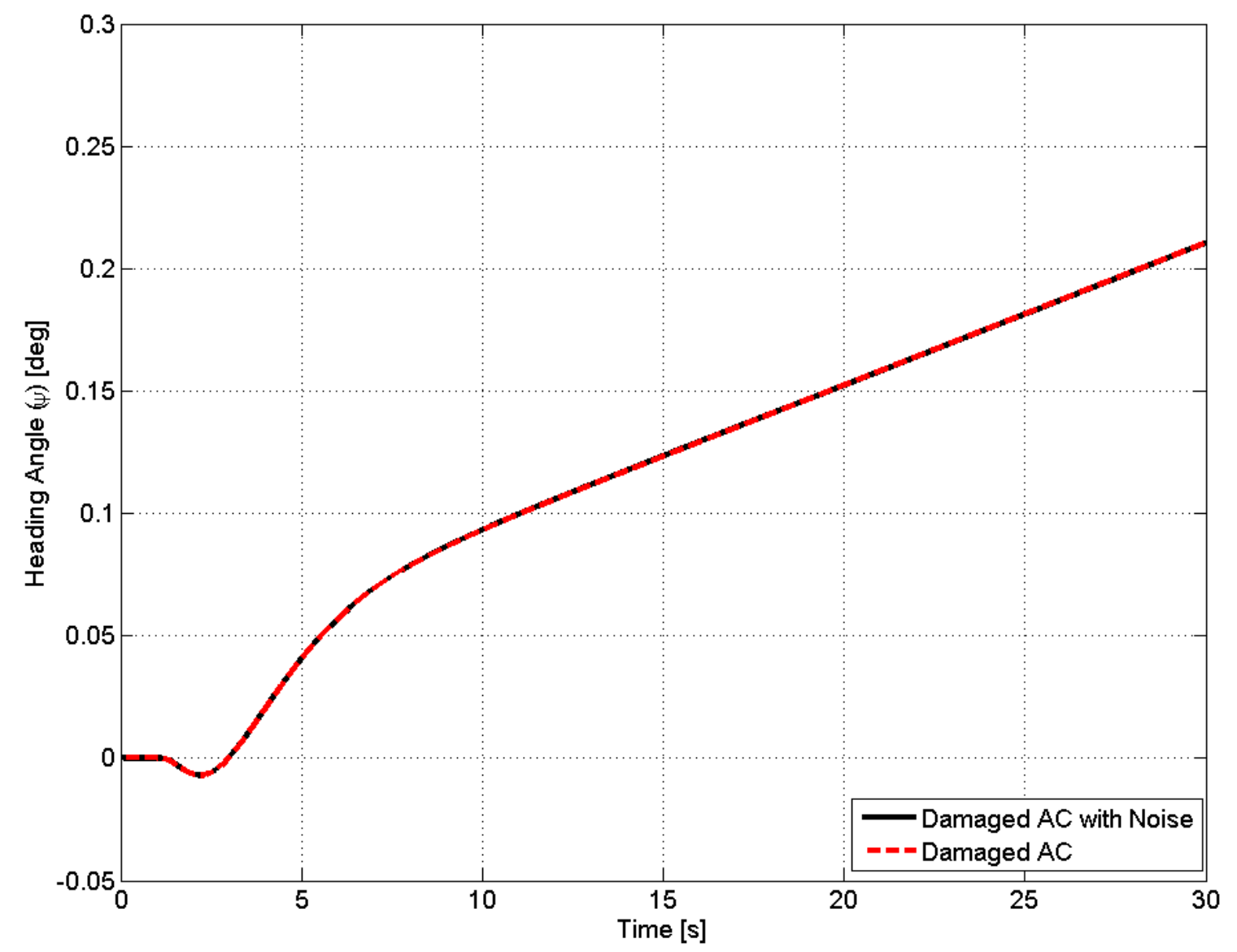

Figure 10.9: Closed loop heading angle response with an LQR controller in the presence of noise 

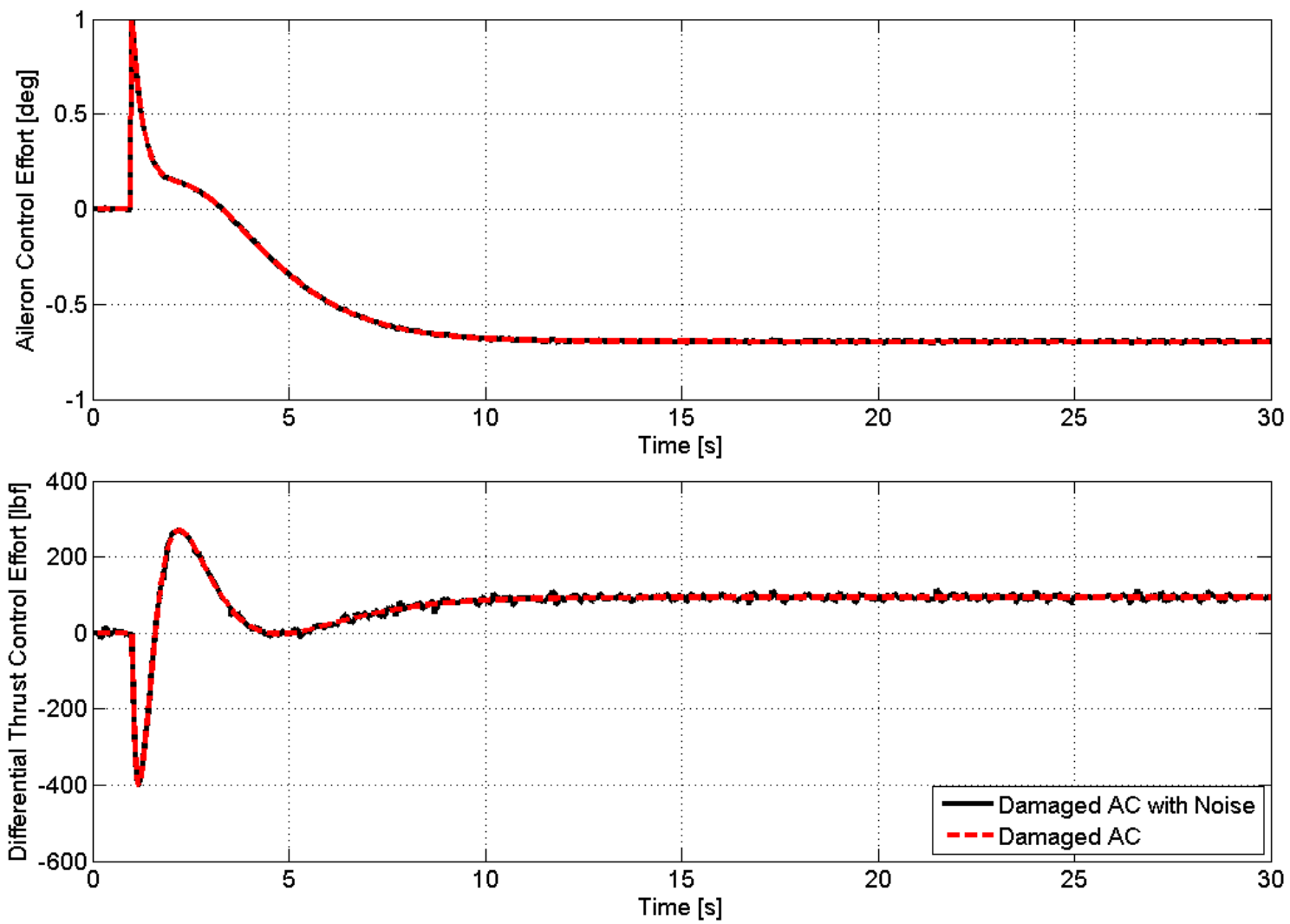

Figure 10.10: Closed loop control efforts with an LQR controller in the presence of noise 
In the presence of noise, the aileron control effort demands the maximum deflection of approximately 1 degree and reaches steady state at around -0.7 degree. The steady state variations are well within bounds for aileron control effort. This aileron control effort demands are reasonable and feasible due to the limiting factors of \pm 26 degrees of the aileron deflection [18] and the assumption that ailerons have instantaneous response characteristics by neglecting the lag from actuators or hydraulic systems.

As for differential thrust, in the presence of noise, the differential thrust control demands at maximum approximately -400 lbf (negative differential thrust means $T 4>T 1$ ), which is within the thrust capability of the JT9D-7A engine, and reaches steady state at around 100 lbf. The steady state variations are also well within bounds for differential thrust control effort. Due to the differential thrust saturation value set at 43,729 $\mathrm{lbf}$ and the thrust rate limiter set at 12,726 lbf/s, this differential thrust control effort in the presence of noise is feasible in a real life situation.

\subsubsection{Robustness and Uncertainty Analysis of LQR Control System}

The robustness of the LQR control system design is studied by the introduction of $30 \%$ of full block, additive uncertainty into the plant dynamics of the damaged aircraft, to test the performance of the damaged aircraft in the presence of uncertainty. Fig. 10.11 shows the logic behind the robustness analysis of the LQR control system design in the presence of uncertainty.

One thousand Monte-Carlo simulations were conducted to test the robustness of the damaged plant in the presence of uncertainty. The state responses in the presence of 30\% uncertainty are shown in Fig. 10.12 and Fig. 10.13.

From Fig. 10.12, it is obvious that the LQR control system design is able to 


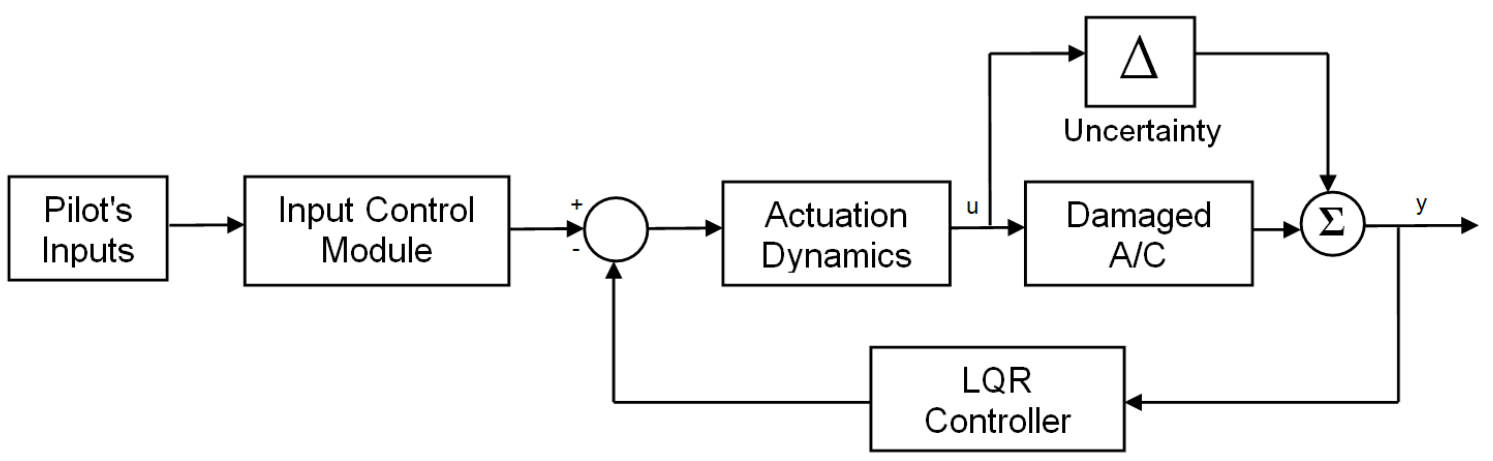

Figure 10.11: Block diagram for full-state feedback LQR control system design in the presence of uncertainty
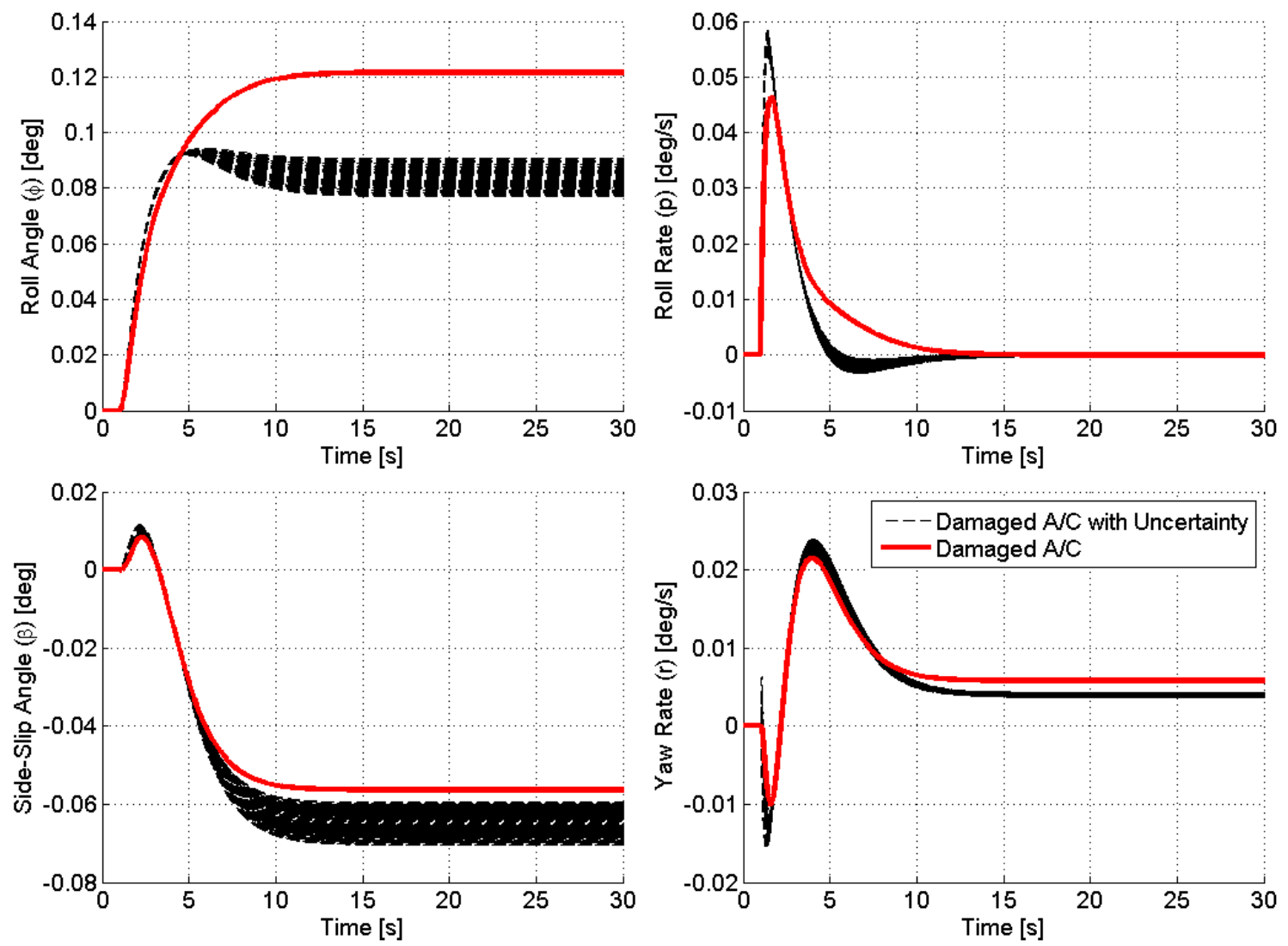

Figure 10.12: Closed loop responses of the damaged aircraft with an LQR controller in the presence of $30 \%$ uncertainty 


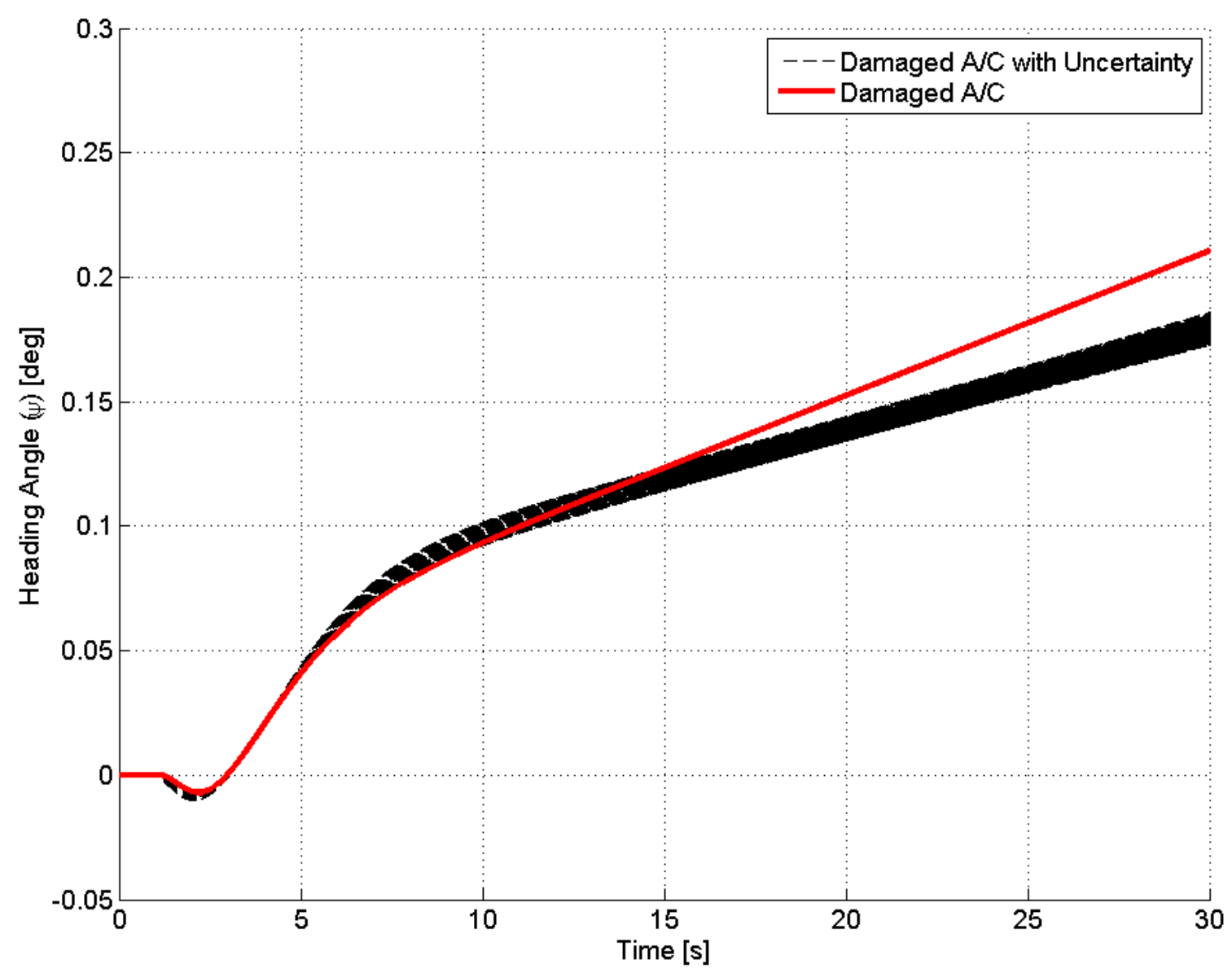

Figure 10.13: Closed loop heading angle response with an LQR controller in the presence of $30 \%$ uncertainty 
stabilize the damaged aircraft within approximately 15 seconds. However, while the roll rate $(p)$ response is still robust and settles at the same point when there is no uncertainty, the steady state responses of three other states: roll angle $(\phi)$, side-slip angle $(\beta)$, and yaw rate $(r)$ all settles at different points compared to when there is no uncertainty. The roll angle $(\phi)$ settles at approximately between 0.080 and 0.090 degree compared to about 0.120 degree when there is no uncertainty . The side-slip angle $(\beta)$ settles at approximately between -0.070 and -0.060 degree compared to about -0.057 degree when there is no uncertainty. Finally, the yaw rate $(r)$ settles at approximately between 0.0036 and $0.0044 \mathrm{deg} / \mathrm{s}$ compared to about $0.0057 \mathrm{deg} / \mathrm{s}$ when there is no uncertainty. As for the heading angle $(\psi)$, the heading angle gain varies between 0.17 and 0.18 degree compared to about 0.22 degree when there is no uncertainty.

Additionally, it is also worth investigating the control efforts required in the presence of uncertainty as shown in Fig. 10.14.

According to Fig. 10.14, when there is $30 \%$ full block, additive uncertainty, the aileron control demands the maximum deflection of approximately 1 degree and reaches steady state at around -0.47 and -0.35 degree after 15 seconds compared to the maximum deflection of 1 degree and settles at around -0.7 degree when there is no uncertainty associated with the damaged aircraft plant. The aileron control effort demands are reasonable and feasible due to the limiting factors of \pm 26 degrees of the aileron deflection [18] and the assumption that ailerons have instantaneous response characteristics by neglecting the lag from actuators or hydraulic systems.

As for differential thrust, when there is $30 \%$ uncertainty, the differential thrust control demands at maximum approximately -1200 lbf (negative differential thrust means $T 4>T 1$ ), which is within the thrust capability of the JT9D-7A engine, and the differential thrust control effort reaches steady state at around the range of -430 

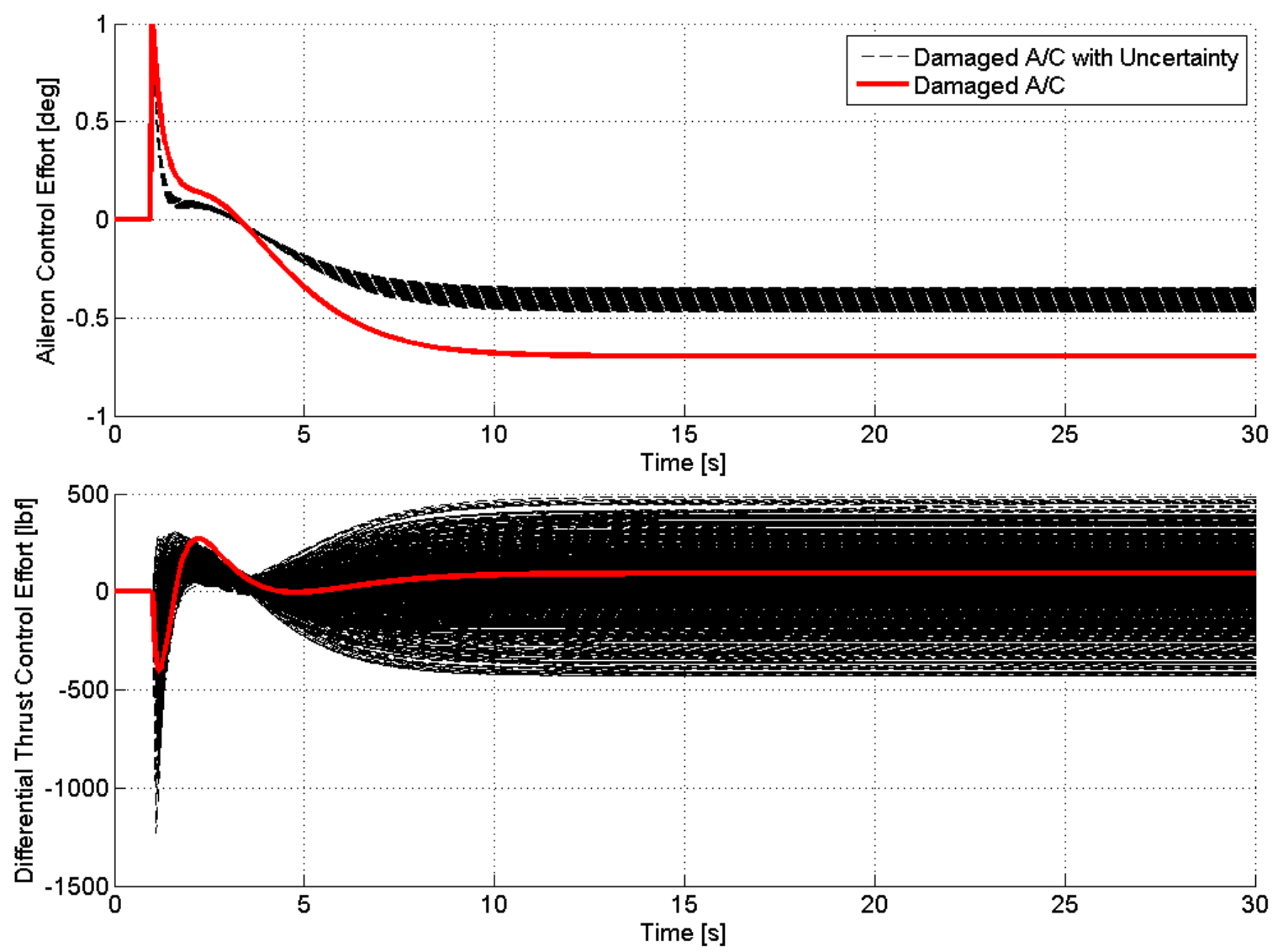

Figure 10.14: Closed loop control efforts with an LQR controller in the presence of $30 \%$ uncertainty 
lbf and $480 \mathrm{lbf}$ after 15 seconds compared to the maximum of $-400 \mathrm{lbf}$ and settles at approximately $100 \mathrm{lbf}$ when there is no uncertainty associated with the damaged aircraft plant. Again, due to the differential thrust saturation value set at 43,729 lbf and the thrust rate limiter set at $12,726 \mathrm{lbf} / \mathrm{s}$, this control effort of differential thrust in the presence of uncertainty is achievable in a real life situation.

\subsection{Chapter Summary}

In this chapter, we have seen that it is possible to stabilize the damaged aircraft with a linear quadratic regulator (LQR) controller. Additionally, as proven in this chapter, although the LQR control system provides the optimal solution to our problem, it does not guarantee robustness in the presence of uncertainty, which is extremely crucial for a damaged aircraft. Therefore, in order to save the damaged aircraft, we will use the LQR controller to stabilize the damaged aircraft (as the inner loop), which will serve as our model aircraft for the adaptive control system design in the next chapter, and utilize the Lyapunov based model reference adaptive control methodology (as the outer loop) to guarantee system stability and robustness. 


\section{CHAPTER 11}

\section{ADAPTIVE CONTROL SYSTEM DESIGN}

To control an aircraft with a fully damaged vertical stabilizer and no rudder is, understandably, a very stressful and laborious task for the pilots. This task also requires skills and experience which are hard to possess and execute in extremely stressful moments. In such instances, pilots usually have seconds to react, and as witnessed beforehand, coupling between the pilot and unstable aircraft dynamics usually led to a catastrophic crash. Therefore, for the safety of the overall flight, it is crucial for an automatic control system to be developed, tested, and implemented for the aircraft to mitigate accidents and to improve safety, stability, and robustness. As an answer to this need, here, we introduce a novel, Lyapunov stability based adaptive control system design.

In conventional model reference adaptive control theory, two celebrated and widely used methods are the MIT rule and the Lyapunov stability approaches [19]. Because of the multi-input-multi-output (MIMO) structure of the lateral/directional dynamics, the MIT rule will not be utilized due to its weak controllability characteristics in higher order and complex systems [19], and the adaptive control system design in this thesis will be based on the celebrated Lyapunov stability approach.

In adaptive control theory, generally speaking, the Lyapunov stability approach is based on the characteristics of a decreasing kinetic energy function of state dynamics. Because the kinetic energy of a system is descending, the system is

considered approaching its asymptotic stability (equilibrium) point. However, it is a 
relatively cumbersome task to derive a kinetic energy function for a complex system, but if a candidate function $V(x)$ could be defined, which represents the characteristics of the kinetic energy function, and if it is descending along the trajectory of the kinetic energy functions, then it can be concluded that the solution

of the governing differential equations $\frac{d y}{d x}=f(x)$ will be asymptotically stable. The function $V(x)$ is then called a Lyapunov function.

\subsection{Stability Characteristics}

Assumption 1. Damaged aircraft control (input) matrix preserves the structure of Eq. (11.1).

$$
B_{d}=\left[\begin{array}{cc}
0 & 0 \\
b_{21} & b_{22} \\
0 & 0 \\
b_{41} & b_{42}
\end{array}\right]
$$

and is assumed to have limited control authority to represent the damaged vertical stabilizer scenario.

It is also worth noting that the structure of Eq. (11.1) is aircraft independent and is preserved by the equations of motion, but not necessarily by any individual aircraft. This means that this is applicable to any damaged aircraft with a dual or quad jet engine lay-out.

Theorem 1. For given system dynamics of the damaged aircraft model in Eq. (4.5-4.8), there exists a Lyapunov function in form of

$$
V(x)=e^{T} P e+\operatorname{Tr}\left[\left(A_{d}-B_{d} L-A_{m}\right)^{T} N\left(A_{d}-B_{d} L-A_{m}\right)\right]
$$


which guarantees asymptotic stability, if and only if the feedback adjustment law is defined as

$$
\dot{L}=\left(B_{d}^{T} N B_{d}\right)^{-1} B_{d}^{T} P e y_{d}^{T}
$$

Proof: Let's consider the suggested Lyapunov function taken from [20],

$$
V(x)=e^{T} P e+\operatorname{Tr}\left[\left(A_{d}-B_{d} L-A_{m}\right)^{T} N\left(A_{d}-B_{d} L-A_{m}\right)\right]+\operatorname{Tr}\left[\left(B_{d} M-B_{m}\right)^{T} R\left(B_{d} M-B_{m}\right)\right]
$$

For given damaged aircraft dynamics, it is desired that aircraft maintains control (input) matrix structure as defined in Assumption 1, leading to $B_{m}=B_{d}$, so in Eq. (11.4), $\operatorname{Tr}\left[\left(B_{d} M-B_{m}\right)^{T} R\left(B_{d} M-B_{m}\right)\right]=0$. Therefore,

$$
V(x)=e^{T} P e+\operatorname{Tr}\left[\left(A_{d}-B_{d} L-A_{m}\right)^{T} N\left(A_{d}-B_{d} L-A_{m}\right)\right]
$$

Here $N$ is the weighting factor, and $\operatorname{Tr}$ is the "Trace" of a matrix. Also, $A_{m}=A_{d}-B_{d} K_{L Q R}$. It is straight-forward that $V(x)>0, \forall x \neq 0, V(0)=0$, and $V(x)$ is continuously differentiable. For given system, error dynamics $\left(e=y_{d}-y_{m}\right)$ become

$$
\begin{aligned}
\dot{e} & =\dot{y_{d}}-\dot{y_{m}} \\
& =\left(A_{d} y_{d}+B_{d} u\right)-\left(A_{m} y_{m}+B_{m} u_{c}\right)
\end{aligned}
$$

With the defined control effort $u=u_{c}-L y_{d}$,

$$
\dot{e}=A_{d} y_{d}+B_{d}\left(u_{c}-L y_{d}\right)-A_{m} y_{m}-B_{m} u_{c}
$$

where $y_{m}=y_{d}-e$. After some algebra, we get

$$
\dot{e}=A_{m} e+\left(A_{d}-B_{d} L-A_{m}\right) y_{d}+\left(B_{d}-B_{m}\right) u_{c}
$$


Again, for given damaged aircraft dynamics, it is desired that aircraft maintains limited control (input) matrix structure as defined in Assumption 1, leading to $B_{m}=B_{d}$. Thus,

$$
\dot{e}=A_{m} e+\left(A_{d}-B_{d} L-A_{m}\right) y_{d}
$$

With $L=L^{*}+\Delta L$ where $L^{*}$ is the constant feedback gain and $\Delta L$ represents the parameter adjustment uncertainty, and $A_{d}-A_{m}=B_{d} L^{*}$,

$$
\dot{e}=A_{m} e+\left(A_{d}-A_{m}-B_{d}\left(L^{*}+\Delta L\right)\right) y_{d}=A_{m} e+\left(B_{d} L^{*}-B_{d} L^{*}-B_{d} \Delta L\right) y_{d}
$$

Therefore,

$$
\dot{e}=A_{m} e-B_{d} \Delta L y_{d}
$$

The derivative of the Lyapunov function from Eq. (11.2) can be obtained as

$$
\dot{V}(x)=-e^{T} Q e+2 \operatorname{Tr}\left[-\Delta L^{T} B_{d}^{T} P e y_{d}^{T}+\Delta L^{T} B_{d}^{T} N B_{d} \Delta \dot{L}\right]
$$

where $A_{m}{ }^{T} P+P A_{m}=-Q$, with positive-definite matrix $Q$ selected to be equal to the Observability Gramian, $C^{T} C$. From here, it is clear that the negative definite nature of Lyapunov function $(\dot{V}(x)<0)$, and therefore, the asymptotic stability of the overall system dynamics is guaranteed when

$$
\Delta L^{T}\left(-B_{d}^{T} P e y^{T}+B_{d}^{T} N B_{d} \Delta \dot{L}\right)=0
$$

is satisfied. This leads to the final adaptation law:

$$
\dot{L}=\left(B_{d}^{T} N B_{d}\right)^{-1} B_{d}^{T} P e y_{d}^{T}
$$

which guarantees the asymptotic stability

\subsection{Adaptive Control System Design}

Before designing an adaptive control system to guarantee adaptive stability of the damaged aircraft and make it behave like the model aircraft, it is crucial to 
check the adaptive law condition: $A_{d}-B_{d} L^{*} \rightarrow A_{m}$ as mentioned in Chapter 11.1. For this study, the model plant is chosen as the stabilized plant of the damaged aircraft by a linear quadratic regulator (LQR) controller, as explained in Chapter 10. The state matrices for the damaged and model aircraft are from Eq. (4.5) and Eq. (10.16), respectively.

$$
\begin{aligned}
A_{d}= & {\left[\begin{array}{cccc}
0 & 1 & 0 & 0 \\
0 & -0.8566 & -2.7681 & 0.1008 \\
0.0478 & 0 & 0 & -1 \\
0 & -0.0248 & 0 & 0
\end{array}\right] } \\
A_{m}=A_{d}-B_{d} K_{L Q R}= & {\left[\begin{array}{cccc}
0 \\
-2.2026 & -3.8851 & -0.5390 & -0.2595 \\
0.0478 & 0 & 0 & -1 \\
-1.4455 & -2.1243 & 8.3210 & -7.8597
\end{array}\right] }
\end{aligned}
$$

Because the linear quadratic regulator (LQR) controller will only change the pole locations and stabilize the plant, the LQR controller does not change the control matrix B. Therefore, it can be clearly seen that

$$
B_{m}=B_{d}=\left[\begin{array}{cc}
0 & 0 \\
0.2249 & 0.0142 \\
0 & 0 \\
0.0118 & 0.6784
\end{array}\right]
$$

Furthermore, the adaptive law condition is satisfied as

$$
A_{d}-B_{d} L^{*}=A_{m}
$$




$$
\begin{gathered}
\rightarrow B_{d} L^{*}=A_{d}-A_{m} \\
\rightarrow L^{*}=B_{d} \backslash\left(A_{d}-A_{m}\right)
\end{gathered}
$$

Here it is also worth noting the pseudo-inverse nature of the damaged aircraft's input matrix $\left(B_{d}\right)$, which is a $4 \times 2$ matrix. The pseudo-inverse of $B_{d}$ can be expressed from the singular value decomposition (SVD) of $B_{d}[21]$.

Let the SVD of $B_{d}$ be

$$
B_{d}=U\left(\begin{array}{cc}
S & 0 \\
0 & 0
\end{array}\right) V^{T}
$$

where $U, V$ are both orthogonal matrices, and $S$ is a diagonal matrix containing the singular values of $B_{d}$.

Then the pseudo-inverse of $B_{d}$ is a $2 \times 4$ matrix defined as

$$
B_{d}^{\dagger}=V\left(\begin{array}{cc}
S^{-1} & 0 \\
0 & 0
\end{array}\right) U^{T}
$$

The pseudo-inverse of the input matrix $B_{d}$ is found. Then, with the information from the $A_{d}$ and $A_{m}$ matrices, the matrix $L^{*}$ can be obtained as

$$
\begin{aligned}
A_{d}-B_{d} L^{*}=A_{m} \rightarrow B_{d} L^{*}=A_{d}-A_{m} \rightarrow L^{*}=\operatorname{pinv}\left(B_{d}\right) *\left(A_{d}-A_{m}\right) \\
\rightarrow L^{*}=\left[\begin{array}{cccc}
9.6697 & 13.2854 & -9.1487 & 0.8729 \\
1.9631 & 2.8644 & -12.1067 & 11.5702
\end{array}\right]
\end{aligned}
$$


Therefore, there exists a matrix $L^{*}$ that will lead the system dynamics to $A_{d}-B_{d} L^{*} \rightarrow A_{m}$, where $A_{m}$ is the model plant dynamic.

The representative block diagram architecture for the suggested adaptive control system design (based on the Lyapunov stability approach) is illustrated in Fig. 11.1. The ultimate goal of the proposed adaptive control system design is to investigate whether the aircraft with a damaged vertical stabilizer is going to be able to mimic model aircraft dynamics and track the responses of the model aircraft or not, by utilizing differential thrust as a control input for lateral/directional dynamics. The control inputs for both plants are one degree step inputs for both the ailerons and differential thrust. It is worth noting that this is an extreme scenario test to see whether the damaged aircraft utilizing differential thrust can hold itself in a continuous yawing and banking maneuver without becoming unstable and losing control.

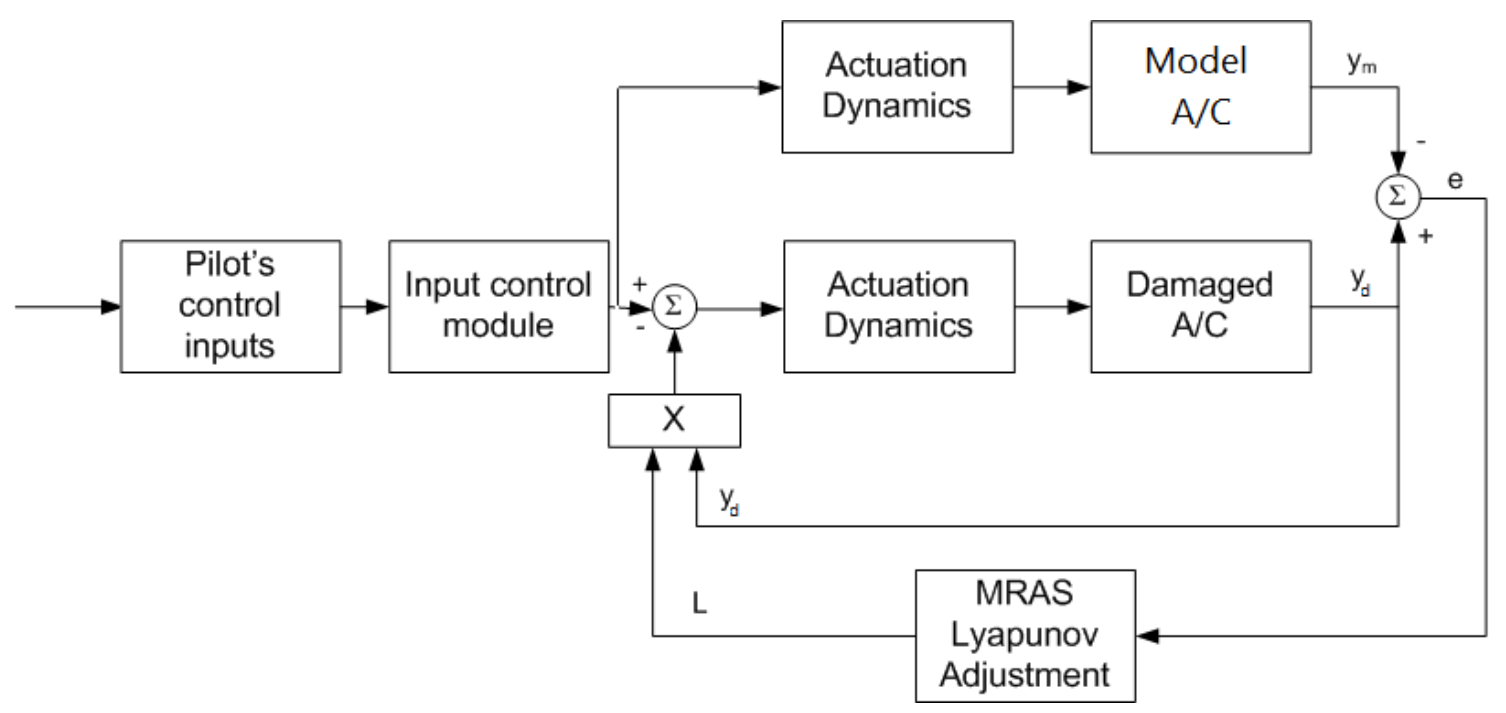

Figure 11.1: Block diagram for adaptive control system design

As seen in Fig. 11.1, the pilot's inputs for both the model and the damaged aircraft, which are one degree step inputs for both ailerons and rudder, will go 
through the Input Control Module, where the aileron control signal is routed through the saturation values of \pm 26 degrees [18] and the rudder control input is routed through the Differential Thrust Control Module, where the rudder input is converted to differential thrust input as discussed previously in Chapter 7 . It is also worth noting that there are also a differential thrust saturation value of 43,729 lbf and a thrust generation rate limiter of $12,726 \mathrm{lbf} / \mathrm{s}$ imposed on the differential thrust control as discussed in Chapter 7. This is to make sure the control inputs are within the limits of both the ailerons and differential thrust. Furthermore, in order for the control efforts to be feasible in a real-life scenario, the control effort signals are also routed through the Actuation Dynamics, where the same saturation values and rate limiters are imposed on the ailerons and differential thrust as discussed previously.

Following to that, the simulation results of the adaptive control system model are presented in Fig. 11.2 and Fig. 11.3. As shown in Fig. 11.2, after only 15 seconds, all four states of the aircraft's lateral/directional dynamics reach steady state values. It can also be clearly seen from Fig. 11.2 and Fig. 11.3 that after a time interval of 15 seconds the damaged aircraft plant can mimic the model aircraft plant, and the heading angles of the damaged and model aircraft also converge at 0.22 degree for one degree step inputs. It is also worth noticing that with the help of differential thrust acting as a "virtual rudder" input during the coordinated turn maneuver, the damaged aircraft was able to follow the behaviors of the model aircraft in achieving very close to zero side-slip angle $(\beta=-0.057 \mathrm{deg})$. Additionally, the errors are minimized as shown in Fig. 11.4. This demonstrates the functionality of the Lyapunov based adaptive control system design in such an extreme scenario.

From Fig. 11.4, it can be observed that the error signals for all four lateral/directional states are diminished after 15 seconds. Therefore, it can be concluded that the damaged aircraft plant can track and mimic an model aircraft in 

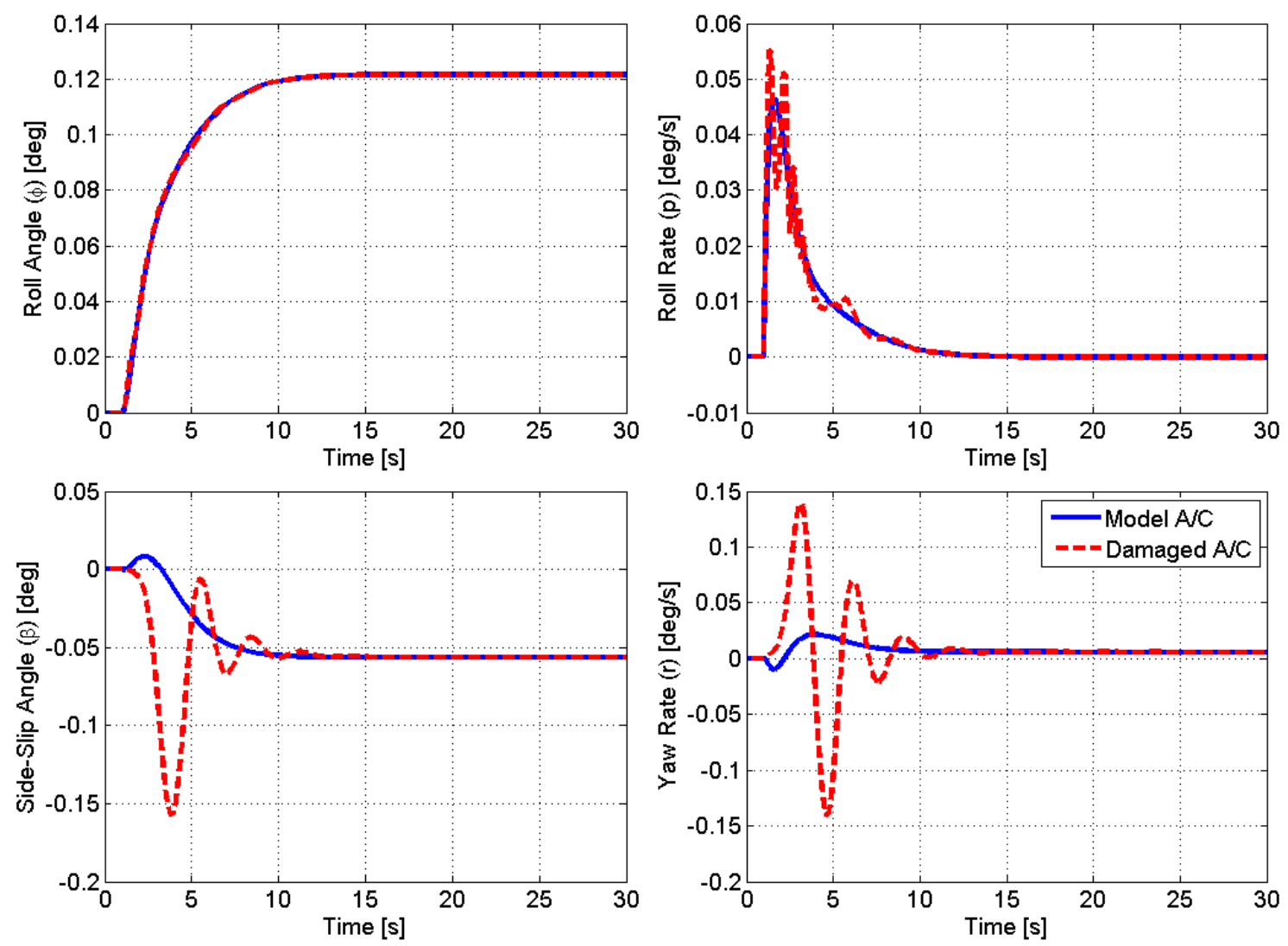

Figure 11.2: Closed loop responses of the damaged aircraft with an adaptive controller 


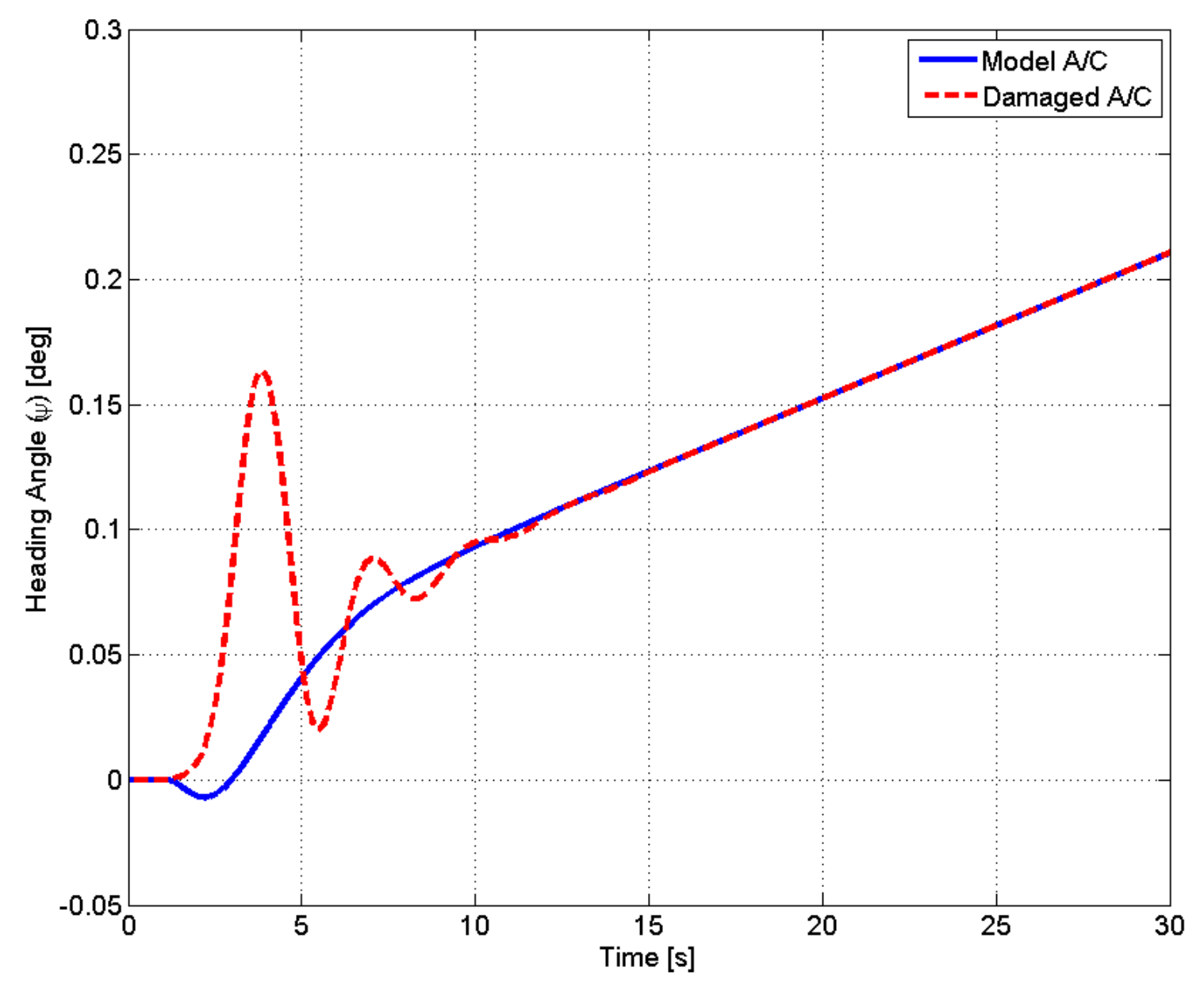

Figure 11.3: Closed loop heading angle response with an adaptive controller 

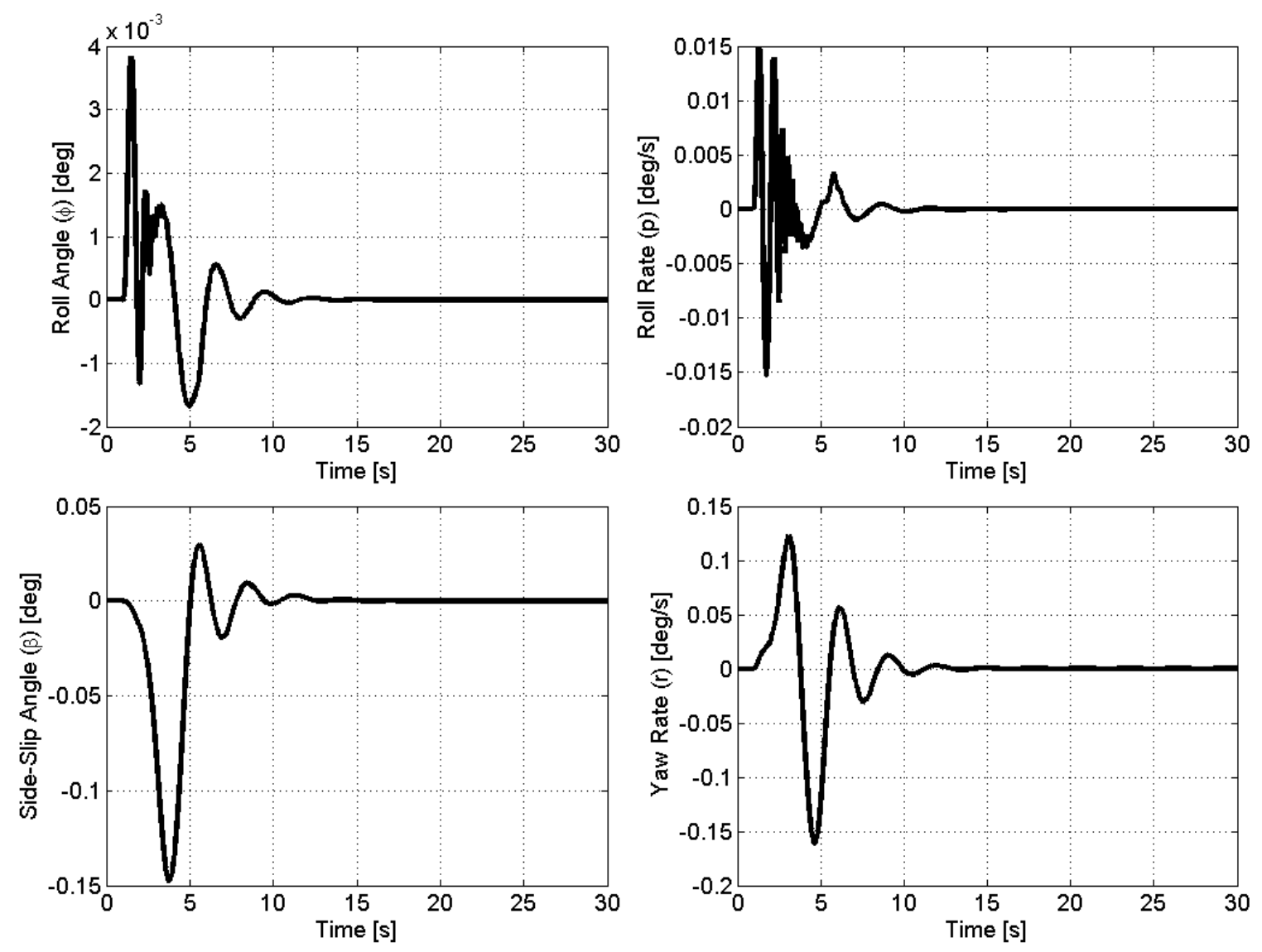

Figure 11.4: Adaptive error signals 
a remarkable fashion. However, this comes at the cost of the control efforts as shown in Fig. 11.5, which are still within control limits and without any saturation of the actuators.
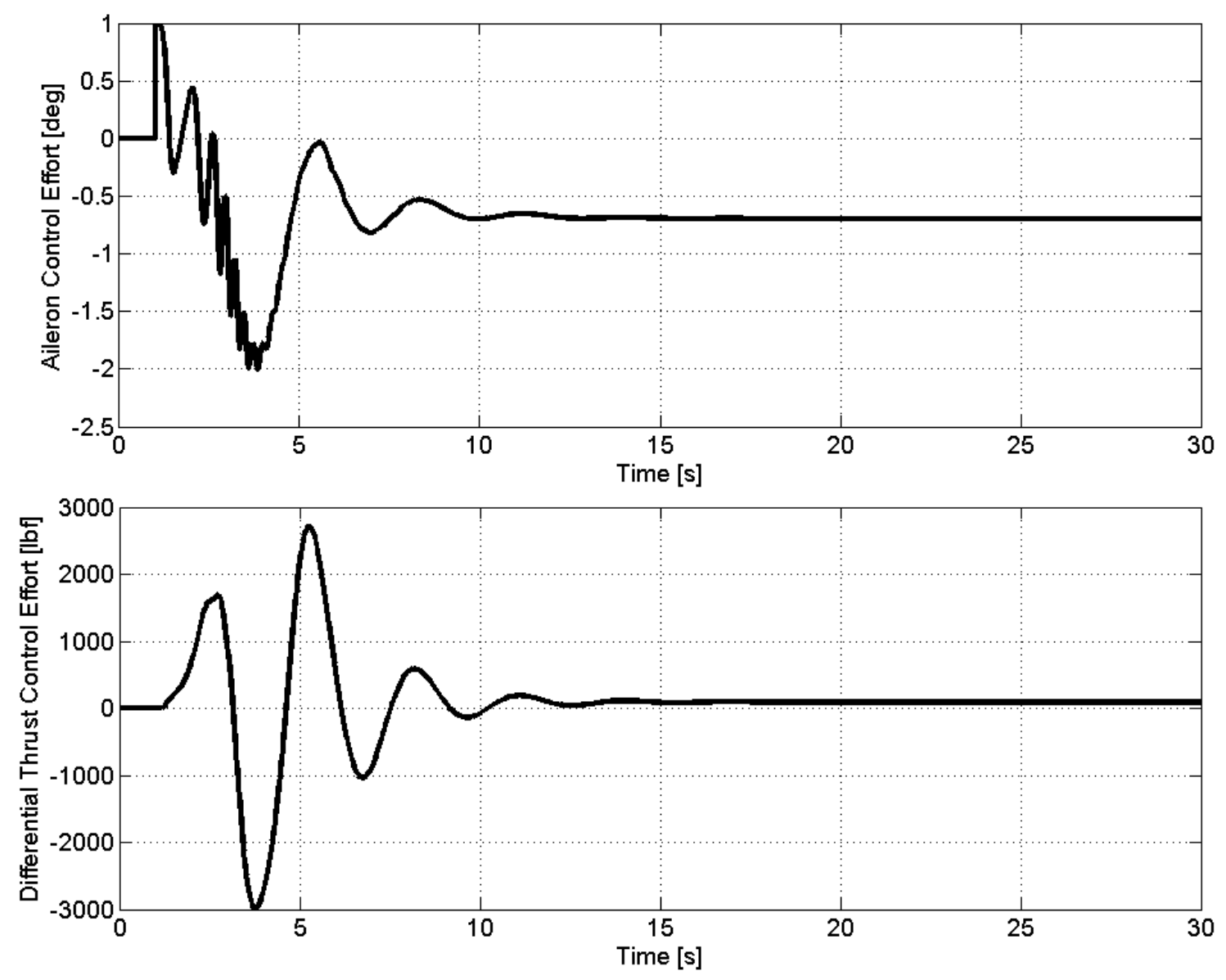

Figure 11.5: Closed loop control efforts with an adaptive controller

In order to have a feasible control strategy in real-life situation, limiting factors are imposed on the aileron and differential thrust control efforts. The aileron deflection is limited at \pm 26 degrees [18]. For differential thrust, a differential thrust saturation value is set at $43,729 \mathrm{lbf}$, which is the difference of the maximum thrust and trimmed thrust values of the JT9D-7A engine. In addition, a rate limiter is also imposed on the thrust response characteristic at 12,726 lbf/s as discussed in 
Chapter 6.

The aileron control effort, as indicated by Fig. 11.5, calls for the maximum deflection of about -2 degrees and reaches steady state at approximately -0.7 degree of deflection after 15 seconds responding to a one degree step input. This aileron control effort is very reasonable and achievable if the ailerons are assumed to have instantaneous response characteristics by neglecting the lag from actuators or hydraulic systems. The differential thrust control effort demands a maximum differential thrust of -3000 lbf (negative differential thrust means $T 4>T 1$ ), which is within the thrust capability of the JT9D-7A engine, and the differential thrust control effort reaches steady state at around 85 lbf after 15 seconds. Therefore, it can be concluded that the adaptive control system design with the utilization of differential thrust as a control input is proven to save the damaged aircraft by making it follow the behaviors of the model aircraft.

\subsection{Robustness and Sensitivity Analysis of Adaptive Control System}

The robustness and sensitivity of the adaptive control system are investigated by the introduction of band-limited white noise and additive uncertainty parameters associated with the state matrix of the damaged aircraft to investigate the performance of the adaptive control system in the presence of noise and uncertainty.

\subsubsection{Noise Sensitivity Analysis of Adaptive Control System}

The noise sensitivity of the adaptive control system presented in this thesis is investigated by the introduction of band-limited white noise to test the performance of adaptive control system in the presence of noise. Fig. 11.6 shows the logic behind the noise sensitivity analysis of the adaptive control system. 


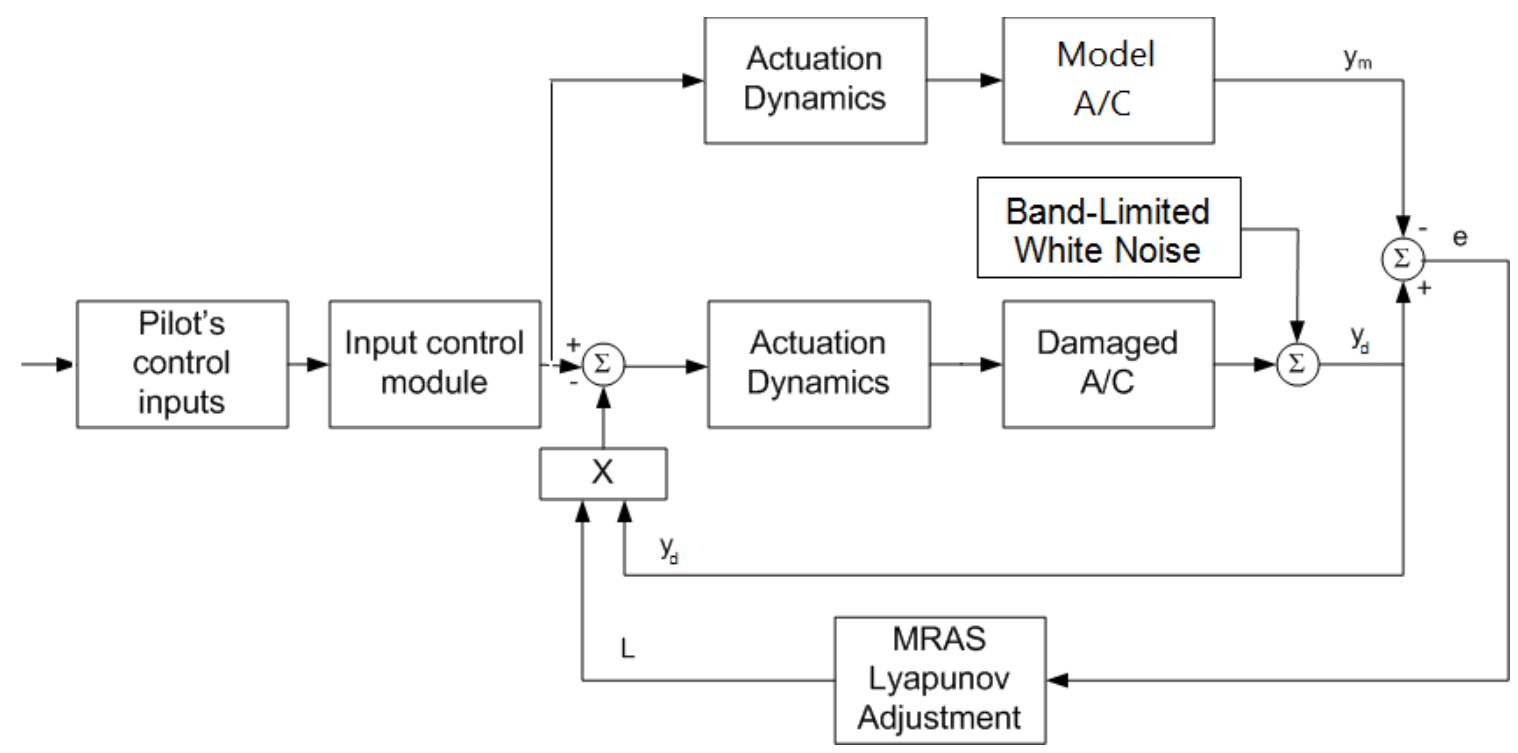

Figure 11.6: Block diagram for adaptive control system design in the presence of noise 
The noise power is set at $10^{-8}$ with sampling time of 0.1 second. The band-limited white noise for the adaptive control system is depicted in Fig. 11.7, and its periodogram using Fast Fourier Transform (FFT) is shown in Fig. 11.8.

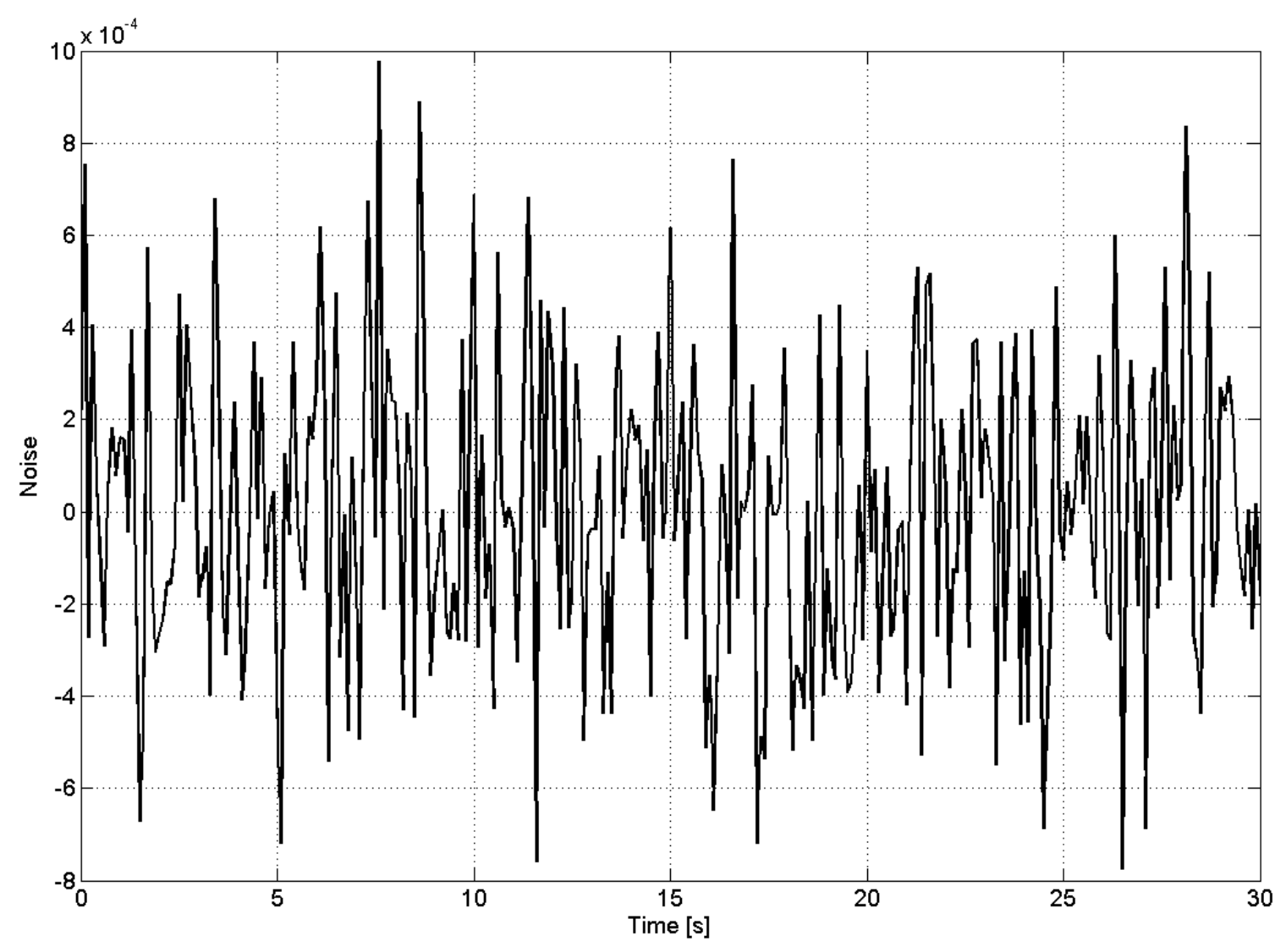

Figure 11.7: Band-limited white noise

Additionally, the closed loop responses of the damaged aircraft with an adaptive controller in the presence of band-limited white noise are shown in Fig. 11.9 and Fig. 11.10. The adaptive error signals in the presence of band-limited white noise are also shown in Fig. 11.11.

From Fig. 11.9, it is obvious that the adaptive control system design is able to stabilize the damaged aircraft within approximately 15 seconds in the presence of noise. We can also see that the roll angle $(\phi)$, side-slip angle $(\beta)$, and yaw rate $(r)$ 


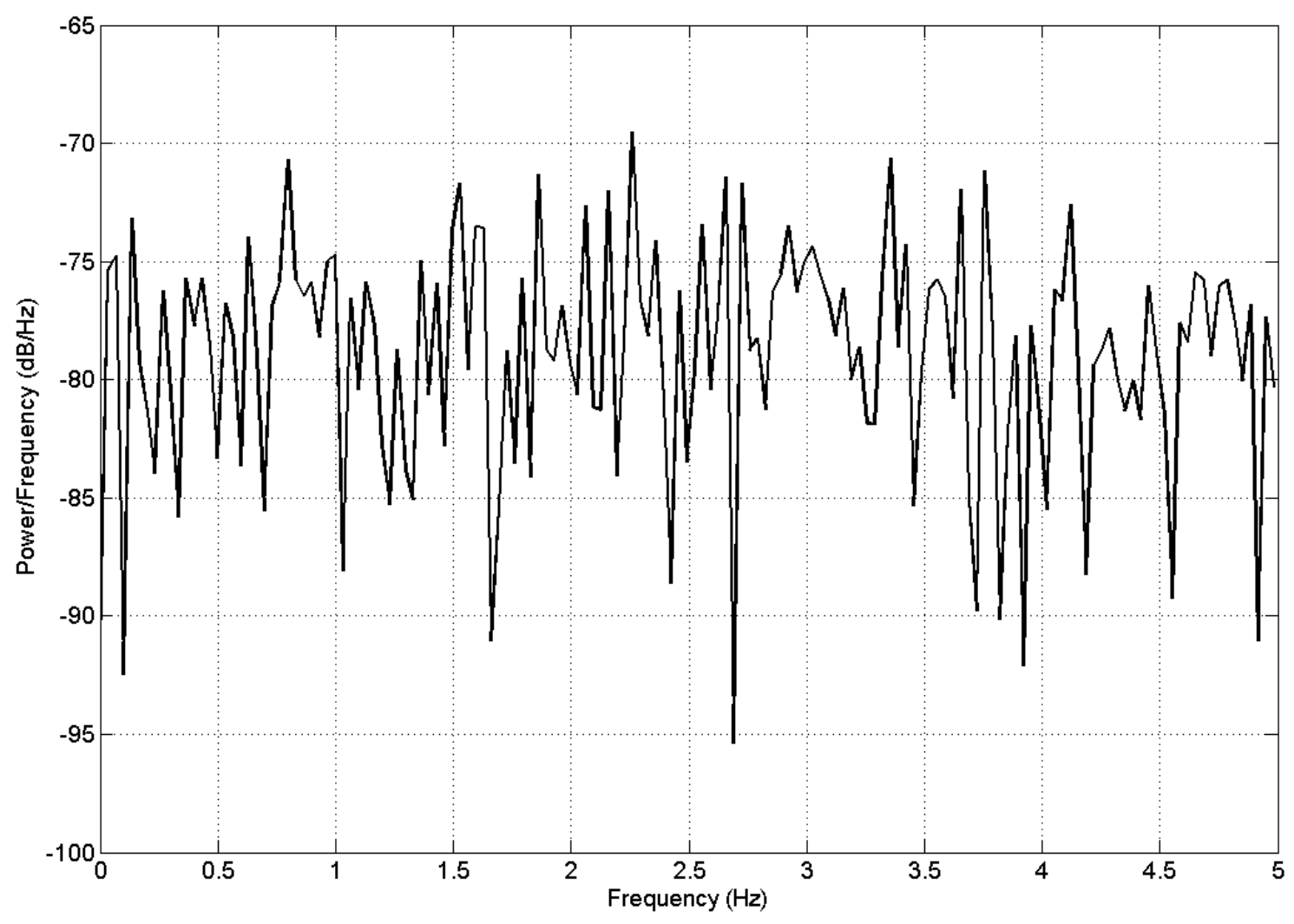

Figure 11.8: Periodogram using Fast Fourier Transform 

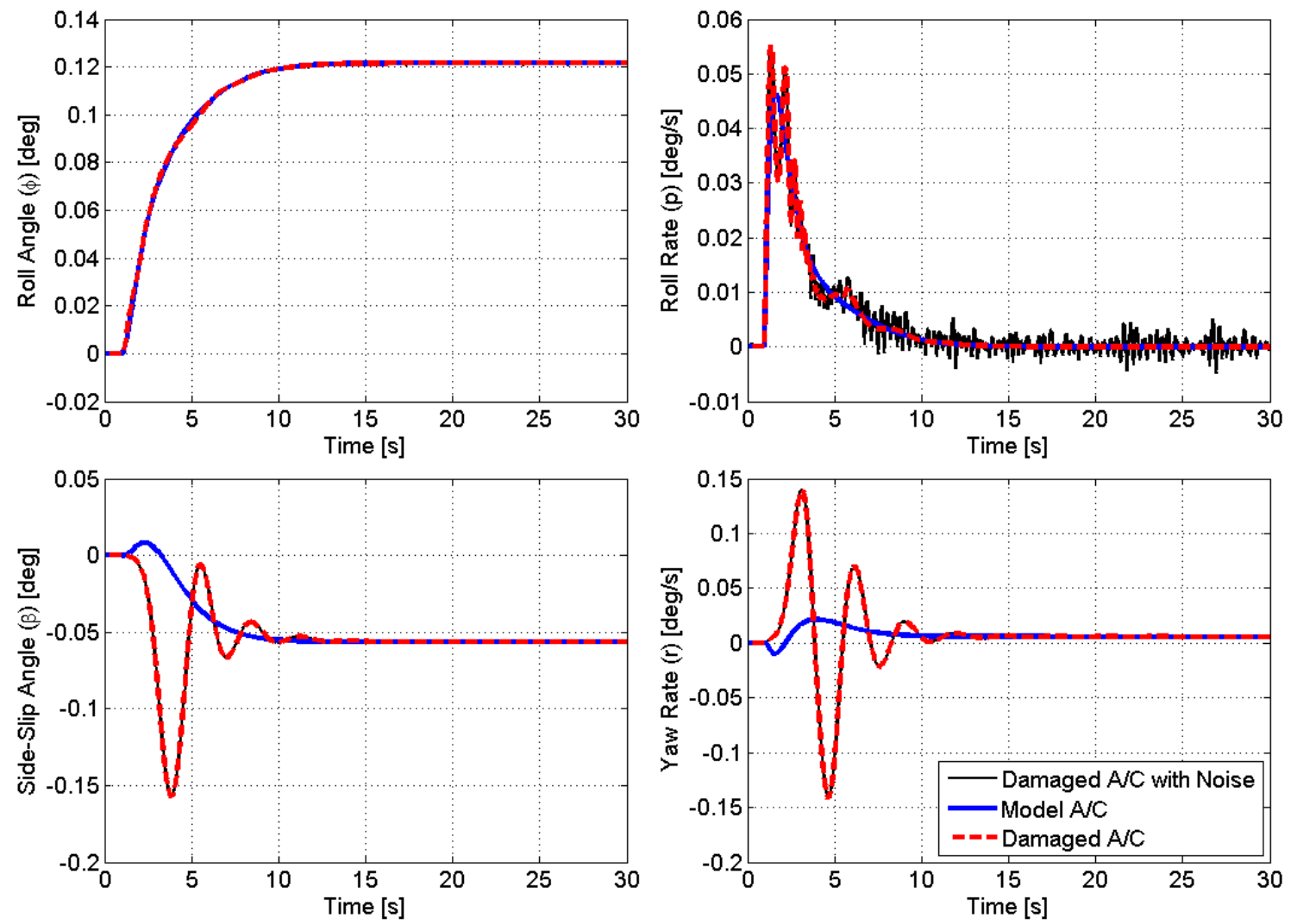

Figure 11.9: Closed loop responses of the damaged aircraft with an adaptive controller in the presence of noise 


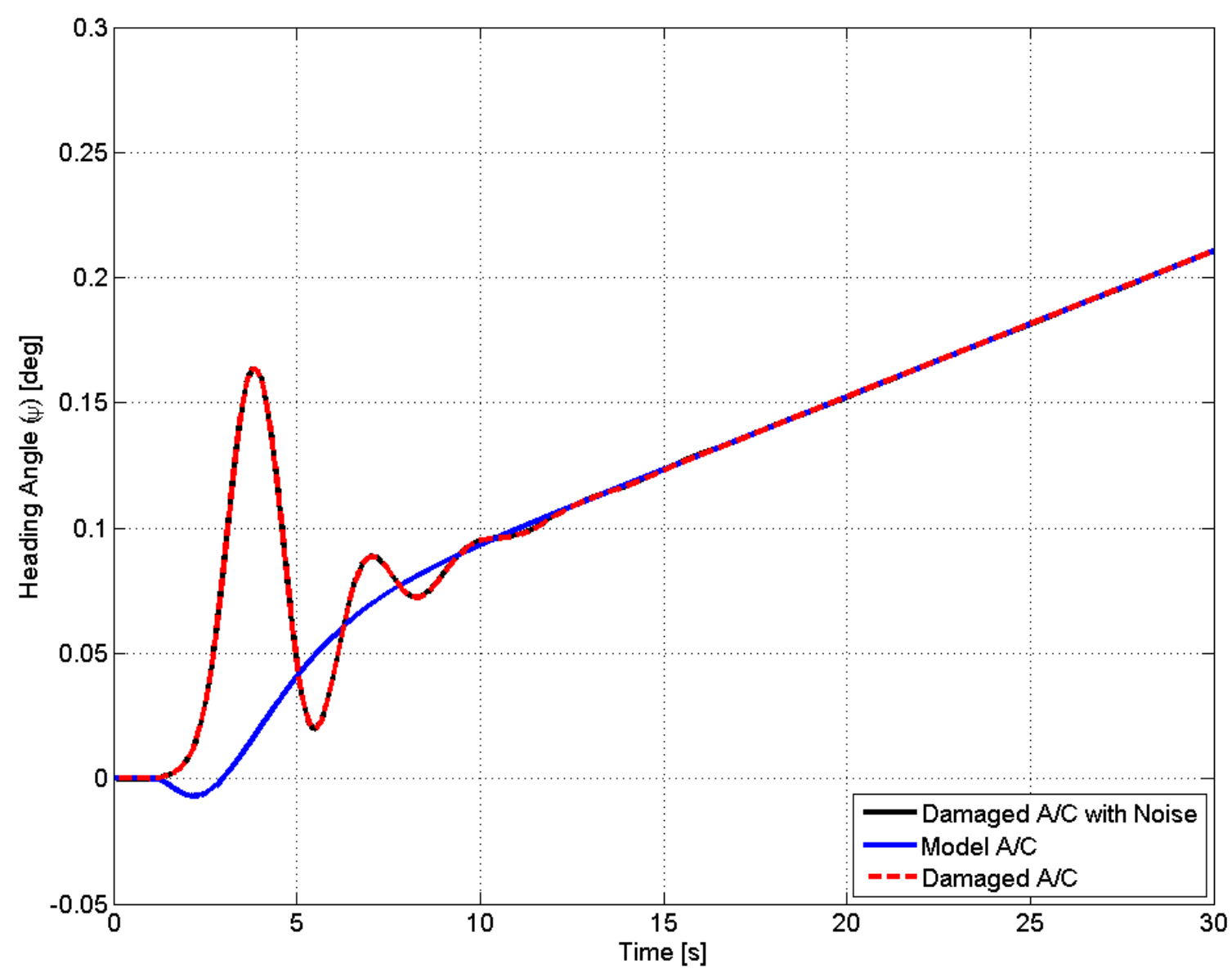

Figure 11.10: Closed loop heading angle response with an adaptive controller in the presence of noise 

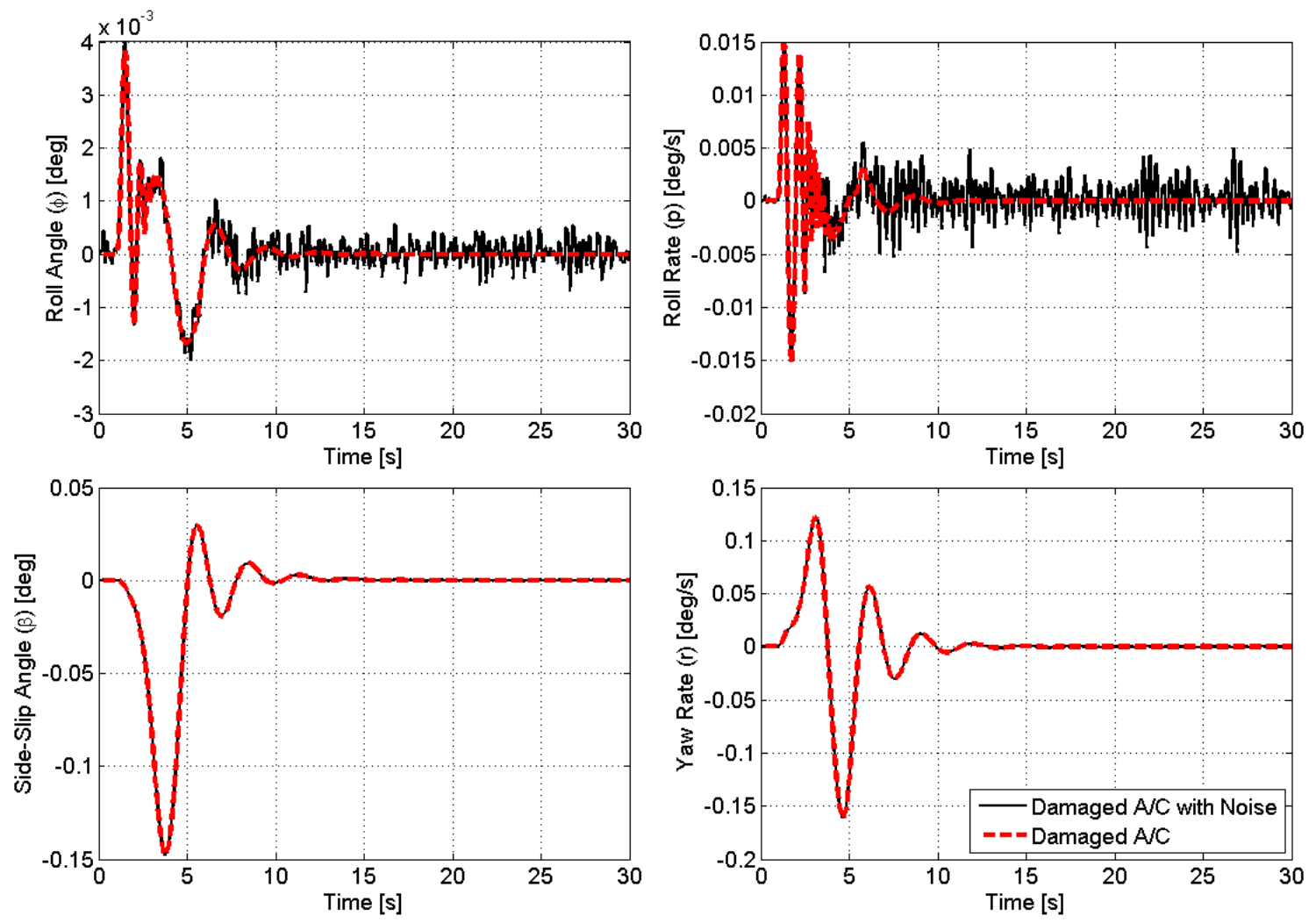

Figure 11.11: Adaptive error signals in the presence of noise 
are least affected by the band-limited white noise. Their responses remain virtually unchanged. However, the roll rate $(p)$ is most affected, and the steady state variations of roll rate associated with noise are also obvious from Fig. 11.9.

As for the heading angle response $(\psi)$ shown in Fig. 11.10, the effect of noise is attenuated by the integral action (because the heading angle $(\psi)$ is the integral of the yaw rate $(r)$ ). The heading angles for the model, damaged, damaged aircraft with noise converge after 15 seconds, and for one degree step inputs, the heading angle gains are about 0.22 degree after 30 seconds.

In addition, from Fig. 11.11, we can see that the adaptive error for slip angle $(\beta)$ and yaw rate $(r)$ are diminished after 15 seconds, but there is still some small steady state variations (in the magnitude of $10^{-3}$ ) for roll angle $(\phi)$ and roll rate $(p)$.

Additionally, it is also worth investigating the control efforts required in the presence of noise as shown in Fig. 11.12.

According to Fig. 11.12, in the presence of noise, the aileron control effort demands the maximum deflection of approximately -2.3 degrees and reaches steady state in the range between -1 and -0.4 degree compared to the maximum aileron control effort of -2 degrees and steady state value of -0.7 degree when there is no noise. This aileron control effort demands in the presence of noise are reasonable and feasible due to the limiting factors of \pm 26 degrees of the aileron deflection [18] and the assumption that ailerons have instantaneous response characteristics by neglecting the lag from actuators or hydraulic systems.

As for differential thrust, in the presence of noise, the differential thrust control effort stays virtually unchanged, which demands at maximum approximately -3000 lbf (negative differential thrust means $T 4>T 1$ ), which is within the thrust capability of the JT9D-7A engine, and reaches steady state at around 85 lbf. Again, due to the differential thrust saturation value set at 43,729 lbf and the thrust rate 

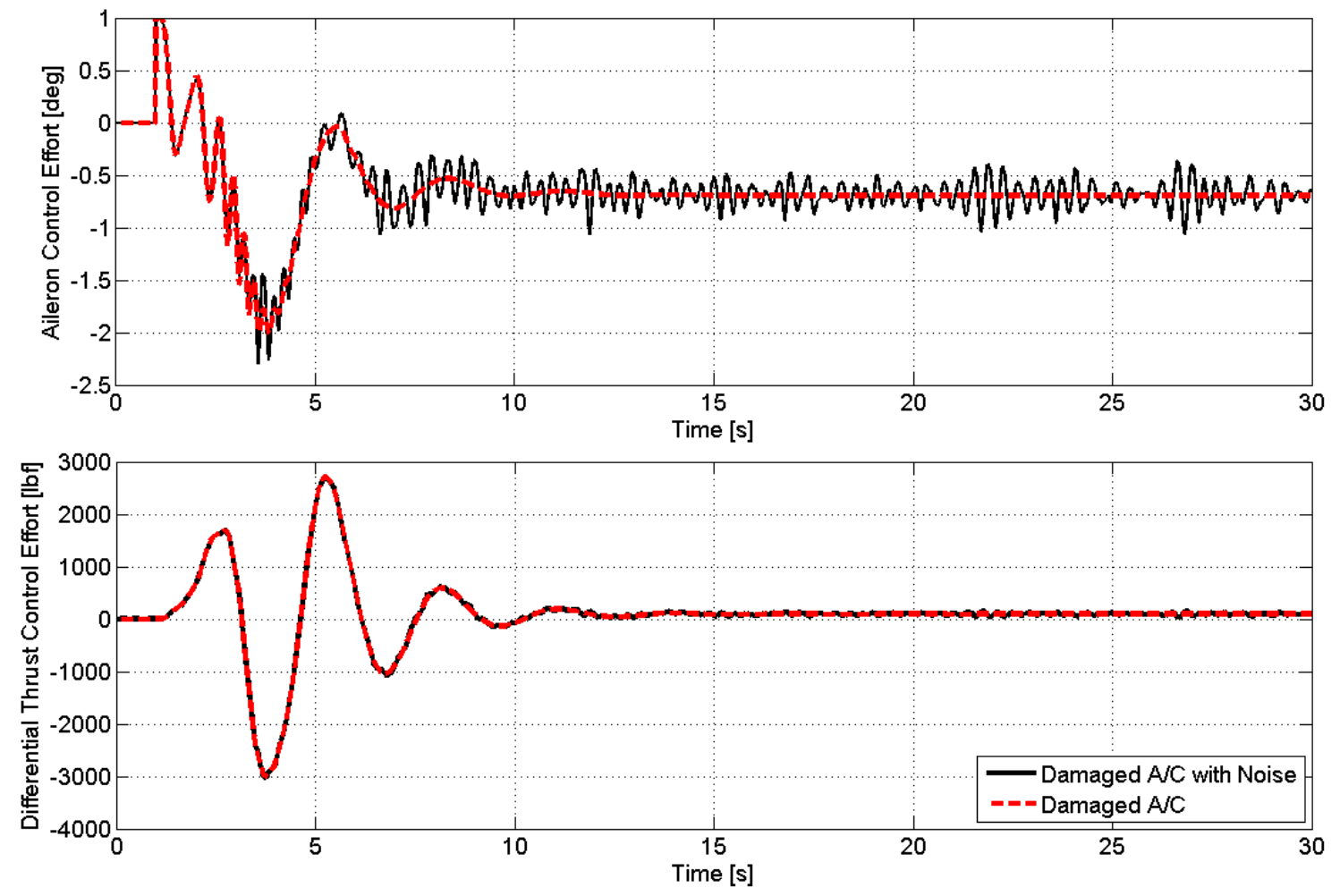

Figure 11.12: Closed loop control efforts with an adaptive controller in the presence of noise 
limiter set at 12,726 lbf/s, this differential thrust control effort in the presence of noise is feasible in a real life situation.

\subsubsection{Robustness and Uncertainty Analysis of Adaptive Control System}

The robustness of the adaptive system design presented in this thesis is investigated by the introduction of $30 \%$ of full block, additive uncertainty into the plant dynamics of the damaged aircraft, to test its ability to track the reference responses of the model aircraft in the presence of uncertainty. Fig. 11.13 shows the logic behind the adaptive control system design in the presence of uncertainty.

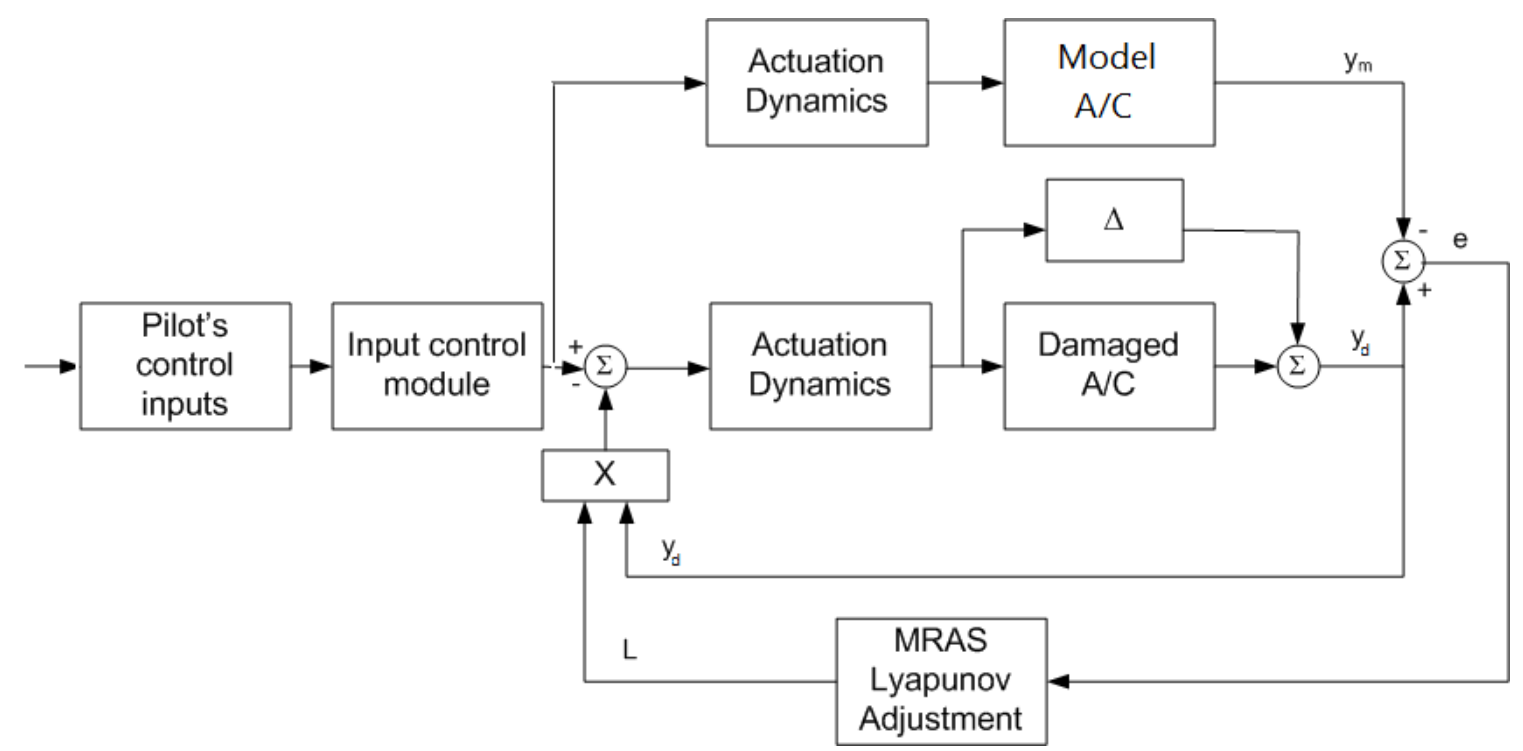

Figure 11.13: Block diagram for adaptive control system design in the presence of uncertainty

One thousand Monte-Carlo simulations were conducted to test the robustness of the damaged plant in the presence of uncertainty. The state responses in the presence of $30 \%$ uncertainty are shown in Fig. 11.14 and Fig. 11.15. It is obvious that the adaptive control system design is able to perform well under given 
uncertain conditions and the damaged aircraft can follow/mimic the responses of the model aircraft only after approximately 15 seconds. In that sense, the uncertain plant dynamics are well within the expected bounds.
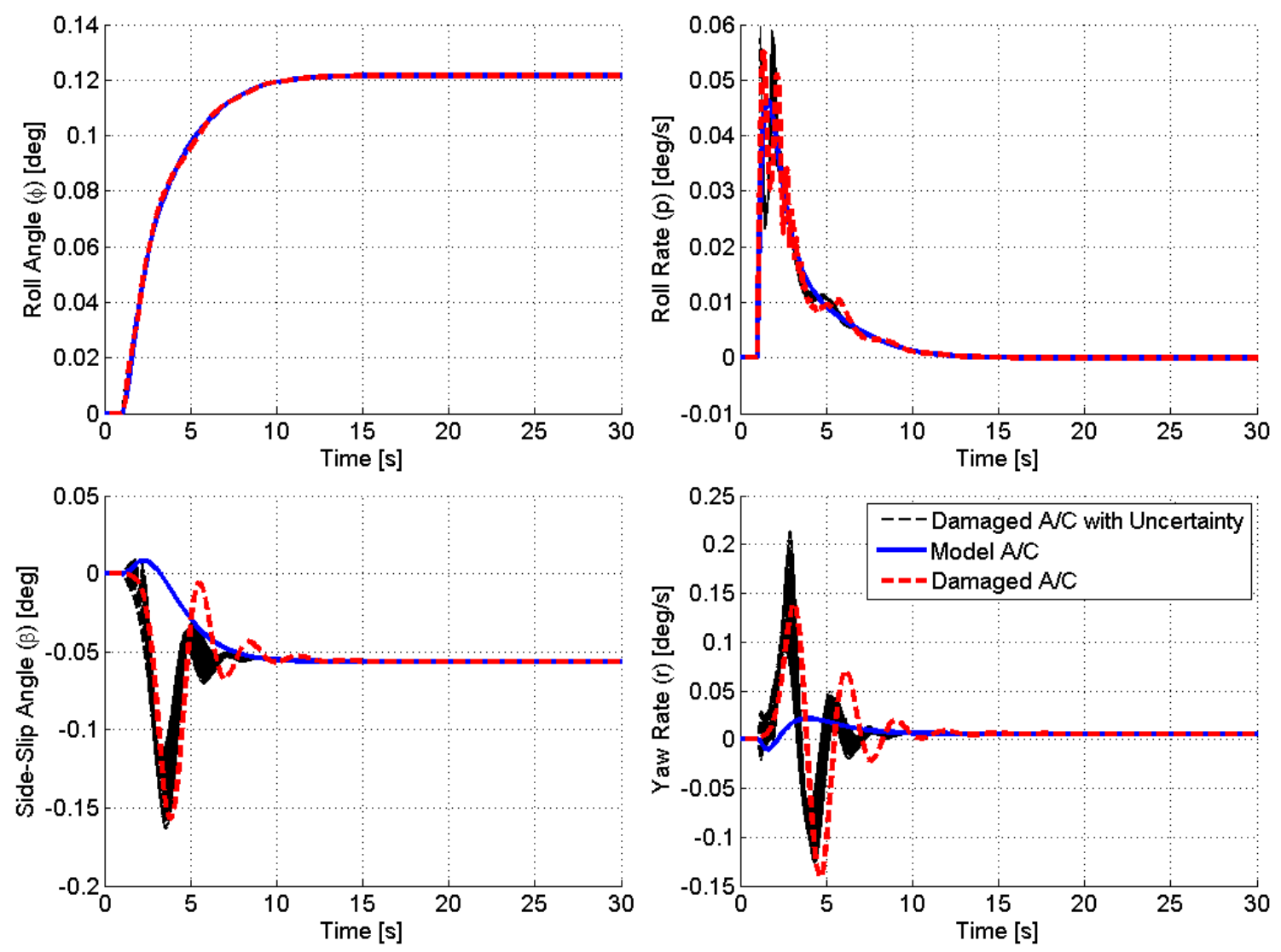

Figure 11.14: Closed loop responses of the damaged aircraft with an adaptive controller in the presence of $30 \%$ uncertainty

The robustness of the adaptive control system design can be further illustrated in Fig. 11.16 that all the error signals reach steady state and converge to zero only after 15 seconds. However, these favorable characteristics come at the expense of the control effort from the ailerons and differential thrust as shown in Fig. 11.17.

According to Fig. 11.17, when there is $30 \%$ full block, additive uncertainty, the aileron control demands the maximum deflection of approximately -1 degree and 


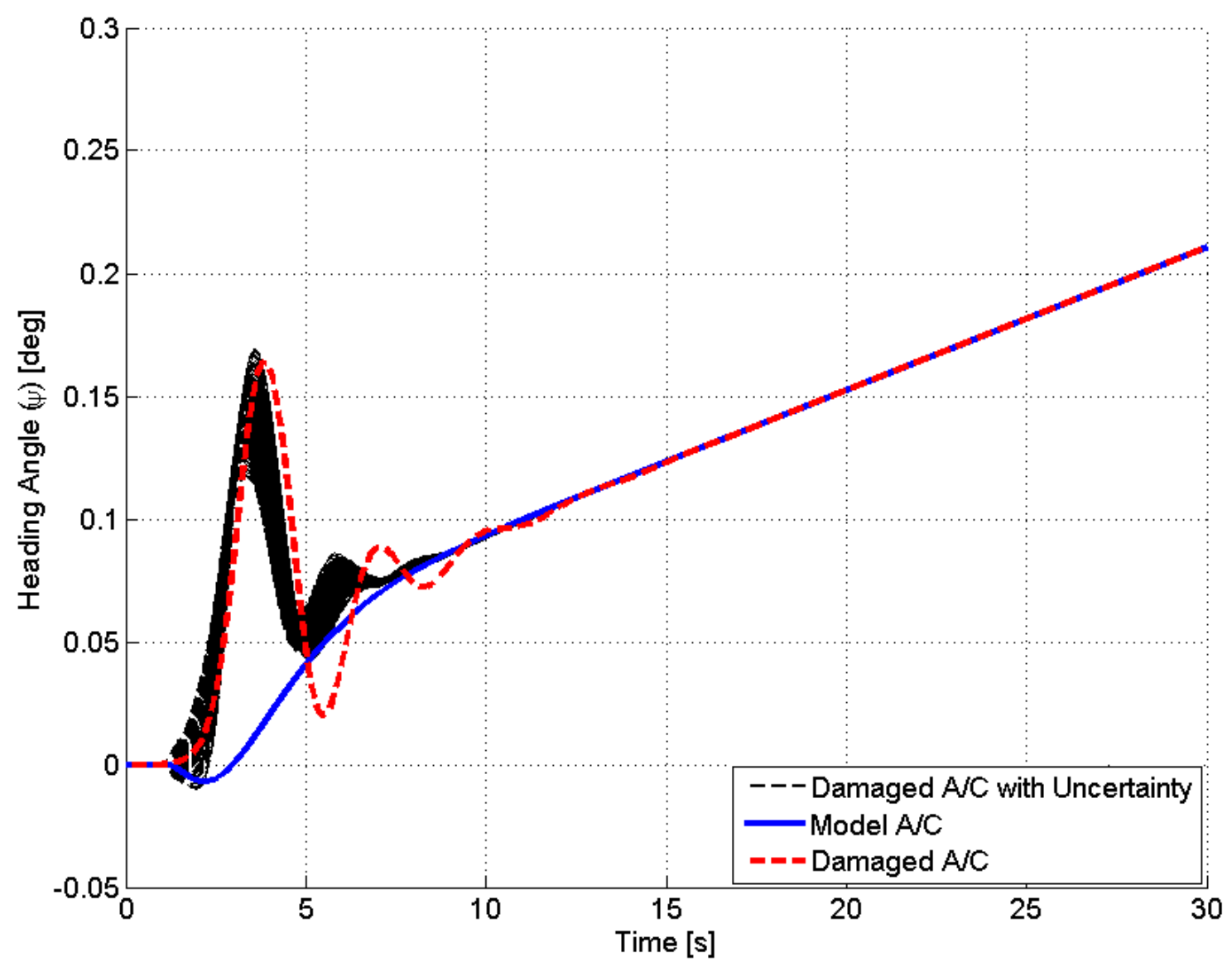

Figure 11.15: Closed loop heading angle response with an adaptive controller in the presence of $30 \%$ uncertainty 

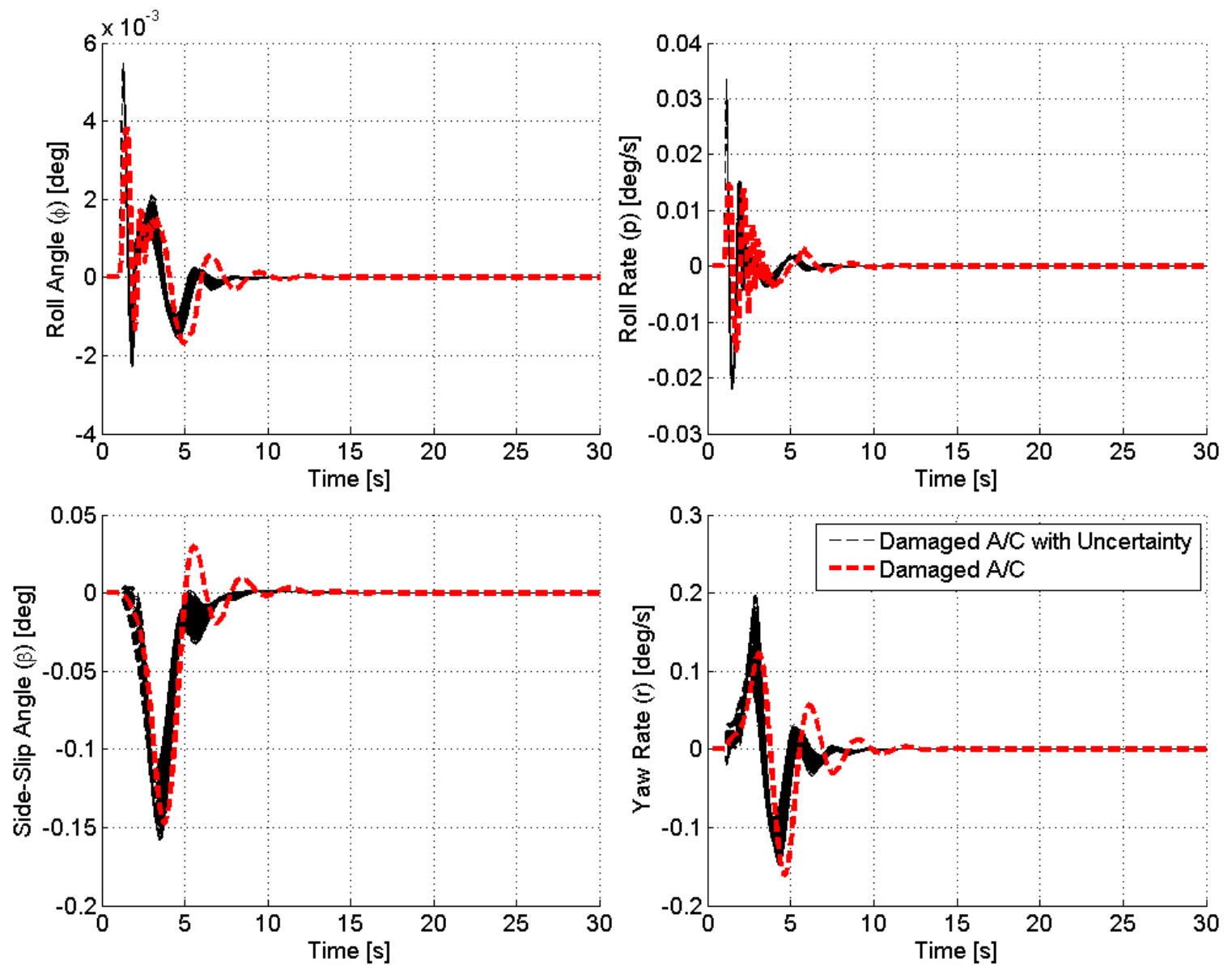

Figure 11.16: Adaptive error signals in the presence of $30 \%$ uncertainty 

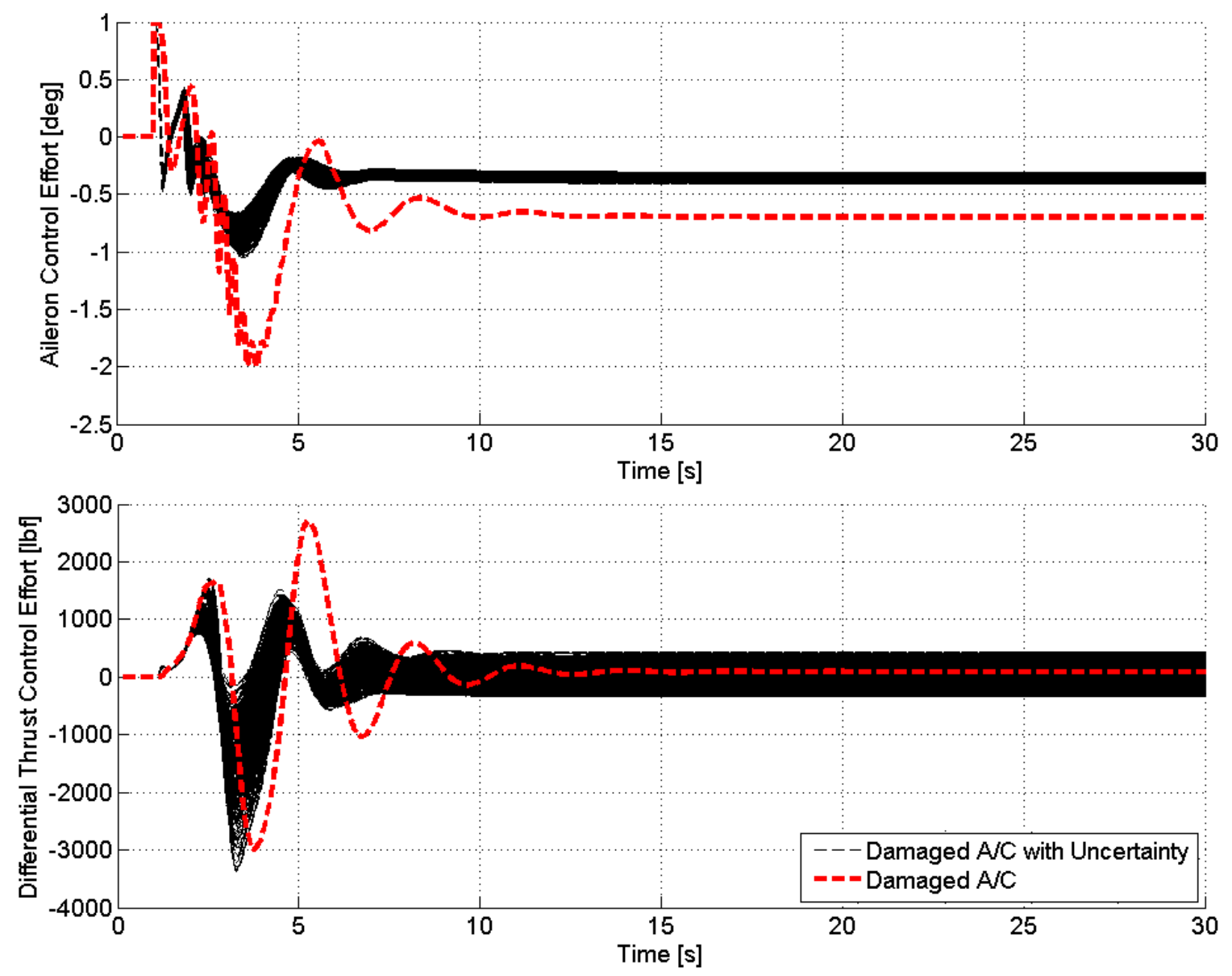

Figure 11.17: Closed loop control efforts with an adaptive controller in the presence of $30 \%$ uncertainty 
reaches steady state at around -0.4 and -0.3 degree after 15 seconds. The aileron control effort demands are reasonable and feasible due to the limiting factors of \pm 26 degrees of the aileron deflection [18] and the assumption that ailerons have instantaneous response characteristics by neglecting the lag from actuators or hydraulic systems.

As for differential thrust, when there is $30 \%$ uncertainty, the differential thrust control demands at maximum approximately -3400 lbf (negative differential thrust means $T 4>T 1$ ), which is within the thrust capability of the JT9D-7A engine, and the differential thrust control effort reaches steady state at around the range of -350 lbf and $450 \mathrm{lbf}$ after 15 seconds. Again, due to the differential thrust saturation value set at 43,729 $\mathrm{lbf}$ and the thrust rate limiter set at 12,726 lbf/s, this control effort of differential thrust in the presence of uncertainty is achievable in a real life situation.

\subsection{Chapter Summary}

In this chapter, we designed an adaptive control system to stabilize the damaged aircraft and make it mimic the behaviors of the model aircraft. We also tested the robustness and sensitivity of the adaptive control system design by introducing band-limited white noise as well as 30\% full block, additive uncertainty into the damaged aircraft plant matrix. It has been concluded that the adaptive control system was able to stabilize the damaged aircraft in only 15 seconds and under feasible control efforts without any actuator saturation. In the next chapter, we are going into designing a robust control system based on the $H_{\infty}$ loop-shaping approach. 


\section{CHAPTER 12}

\section{$H_{\infty}$ LOOP-SHAPING BASED ROBUST CONTROL SYSTEM DESIGN}

\section{1 $\quad H_{\infty}$ Loop-Shaping Based Robust Control System Design}

As seen in Chapter 8, the open loop responses of the damaged aircraft are unstable in all four lateral/directional states. This means the pilot will not have much chance to save the aircraft, which calls for a novel approach to save the damaged aircraft and to provide a safe landing. In addition, in Chapter 11, we designed a model reference adaptive control system to stabilize and save the damaged aircraft by making it follow and mimic the behaviors of the model aircraft. However, for the model reference adaptive control system, there is a need to design a model plant before designing the control system itself. In this chapter, we want to emphasize the need and necessity of designing a robust controller to stabilize and save the damaged aircraft without the need to develop the model plant. Our goal is to design a feedback controller which will not only stabilize the plant but which will also make it robust. Therefore, the $H_{\infty}$ loop-shaping based robust control system is chosen as a means to stabilize the damaged aircraft due to its ability to suppress external disturbances and overall system robustness.

The damaged aircraft plant's open loop singular values are shaped by the preand post-compensation [22] as illustrated in Fig. 12.1.

As seen in Fig. 12.1, $G$ is the open loop plant of the damaged aircraft while $W_{1}$ and $W_{2}$ are the pre- and post-compensation, respectively. The shaped plant is, therefore, as shown in Eq. (12.1). 


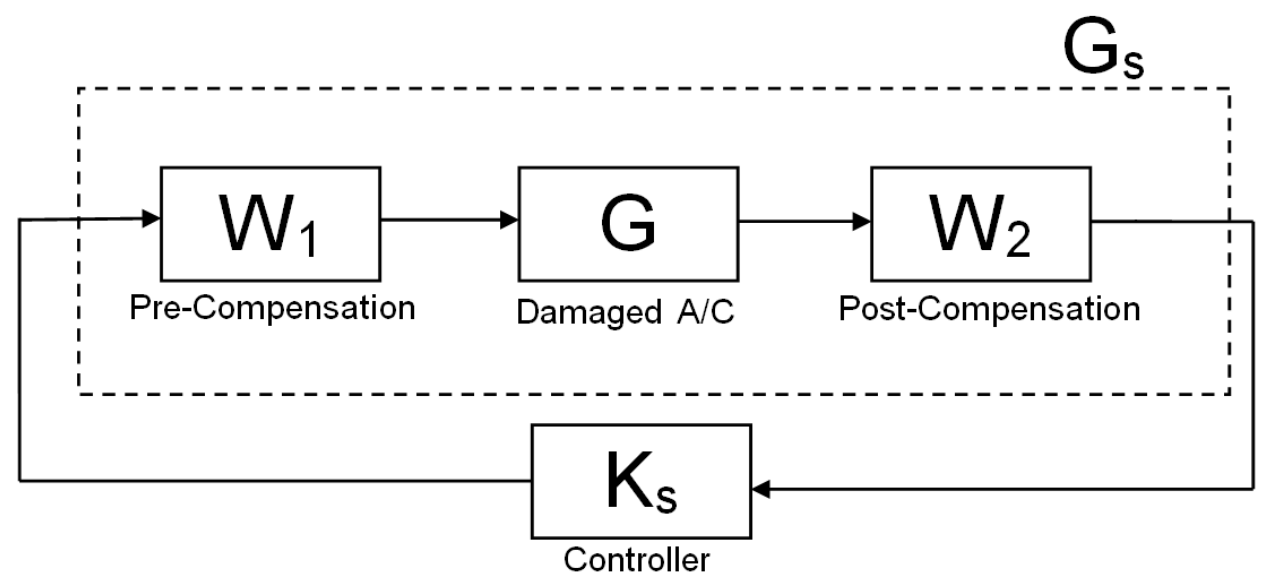

Figure 12.1: The damaged aircraft's shaped plant and controller 


$$
G_{s}=W_{2} G W_{1}
$$

In addition, the controller $\left(K_{s}\right)$ is synthesized by solving the robust stabilization problem for the shaped plant $\left(G_{s}\right)$ with a normalized left coprime factorization of $G_{s}=M_{s}{ }^{-1} N_{s}$, and the feedback controller for plant $G$ is, therefore, $K=W_{1} K_{s} W_{2}[23]$.

Next, the implemented $H_{\infty}$ loop-shaping diagram is shown in Fig. 12.2.

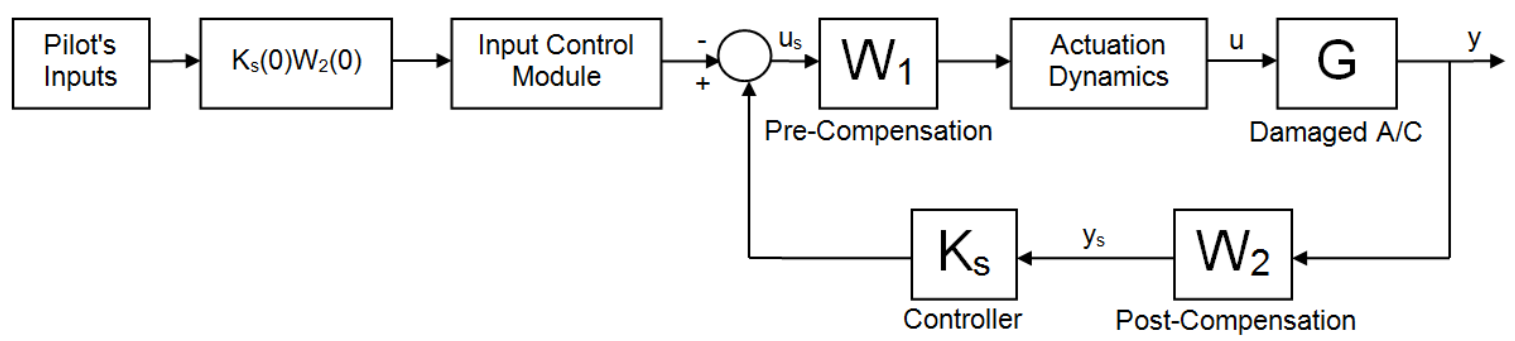

Figure 12.2: Block diagram for $H_{\infty}$ loop-shaping based robust control system design

Figure 12.2 provides an insight into the robust controller design based on the $H_{\infty}$ loop-shaping approach. The main goal of this controller is to stabilize all four lateral/directional states of the damaged aircraft, which are roll rate $(p)$, roll angle $(\phi)$, side-slip angle $(\beta)$, and yaw rate $(r)$. The control inputs of the damaged aircraft are ailerons $\left(\delta_{a}\right)$ and differential thrust $(\delta T)$. As seen in Fig. 12.2, the pilot's inputs, which are one degree step inputs for both ailerons and rudder, will go through the pre-filter gain $K_{s}(0) W_{2}(0)$ and then the Input Control Module, where the aileron control signal is routed through the saturation values of \pm 26 degrees [18] and the rudder control input is routed through the Differential Thrust Control Module, where the rudder input is converted to differential thrust input as discussed previously in Chapter 7. It is also worth noting that there are also a differential thrust saturation value of 43,729 lbf and a thrust generation rate limiter 
of $12,726 \mathrm{lbf} / \mathrm{s}$ imposed on the differential thrust control as discussed in Chapter 7. This is to make sure the control inputs are within the limits of both the ailerons and differential thrust. Furthermore, in order for the control efforts to be feasible in a real-life scenario, the control effort signals are also routed through the Actuation Dynamics, where the same saturation values and rate limiters are imposed on the ailerons and differential thrust as discussed previously.

The next step is to select the weighting functions, which should be taken into careful consideration due to the dimensions of the system. Unfortunately, for most of the time, the system matrices that represent dynamical systems are non-square. Therefore, the selection of weighting functions requires a simple dimensional analysis. The damaged aircraft's lateral/directional system is [4x2], which represents 4 outputs and 2 inputs. Due to Eq. (12.1), which states $G_{s}=W_{2} G W_{1}$, the dimension of $W_{2}$ needs to be [4x4] signifying a system of 4 outputs and 4 inputs, and the dimension of $W_{1}$ needs to be [2x2], representing a system of 2 outputs and 2 inputs.

After an iterative process, the selected weighting functions for $W_{1}$ are as

$$
\begin{gathered}
W_{1(11)}=\frac{4 s+1}{4 s+10} \\
W_{1(22)}=\frac{50 s+5}{18 s+25}
\end{gathered}
$$

We can then construct the system matrix of $W_{1}$ as 


$$
W_{1}=\left[\begin{array}{ccccc}
-2.5000 & 0 & 2.5981 & 0 & 2 \\
0 & -1.3889 & 0 & 1.8922 & 0 \\
-2.5981 & 0 & 3 & 0 & 0 \\
0 & -1.8922 & 0 & 2.7778 & 0 \\
0 & 0 & 0 & 0 & -\infty
\end{array}\right]
$$

The selected weighting functions for $W_{2}$ are as

$$
\begin{gathered}
W_{2(11)}=\frac{16}{s+16} \\
W_{2(22)}=W_{2(33)}=W_{2(44)}=\frac{120}{s+120}
\end{gathered}
$$

where the system matrix of $W_{2}$ becomes

$$
W_{2}=\left[\begin{array}{ccccccccc}
-16 & 0 & 0 & 0 & 4 & 0 & 0 & 0 & 4 \\
0 & -120 & 0 & 0 & 0 & 10.9545 & 0 & 0 & 0 \\
0 & 0 & -120 & 0 & 0 & 0 & 10.9545 & 0 & 0 \\
0 & 0 & 0 & -120 & 0 & 0 & 0 & 10.9545 & 0 \\
4 & 0 & 0 & 0 & 0 & 0 & 0 & 0 & 0 \\
0 & 10.9545 & 0 & 0 & 0 & 0 & 0 & 0 & 0 \\
0 & 0 & 10.9545 & 0 & 0 & 0 & 0 & 0 & 0 \\
0 & 0 & 0 & 10.9545 & 0 & 0 & 0 & 0 & 0 \\
0 & 0 & 0 & 0 & 0 & 0 & 0 & 0 & -\infty
\end{array}\right]
$$

As it is customary in general loop-shaping formulation, the maximum stability margin $\left(e_{\max }\right)$ is defined as a performance criterion:

$$
e_{\max }=1 / \gamma_{\min }
$$


where $\gamma_{\min }$ is the $H_{\infty}$ optimal cost. For our analysis, the maximum stability margin is then obtained as

$$
e_{\max }=0.2763
$$

which is within the suggested optimal value bounds: $0.25<e_{\max }<0.30$.

Next, the sensitivity (S) and co-sensitivity (T) plots are constructed, where the sensitivity $(\mathrm{S})$ function is defined as the transfer function from the disturbance signal to the output while the co-sensitivity $(\mathrm{T})$ function is defined as the transfer function from the reference signal to the output. The input and output sensitivity functions are shown in Fig. 12.3 and Fig. 12.4, respectively.

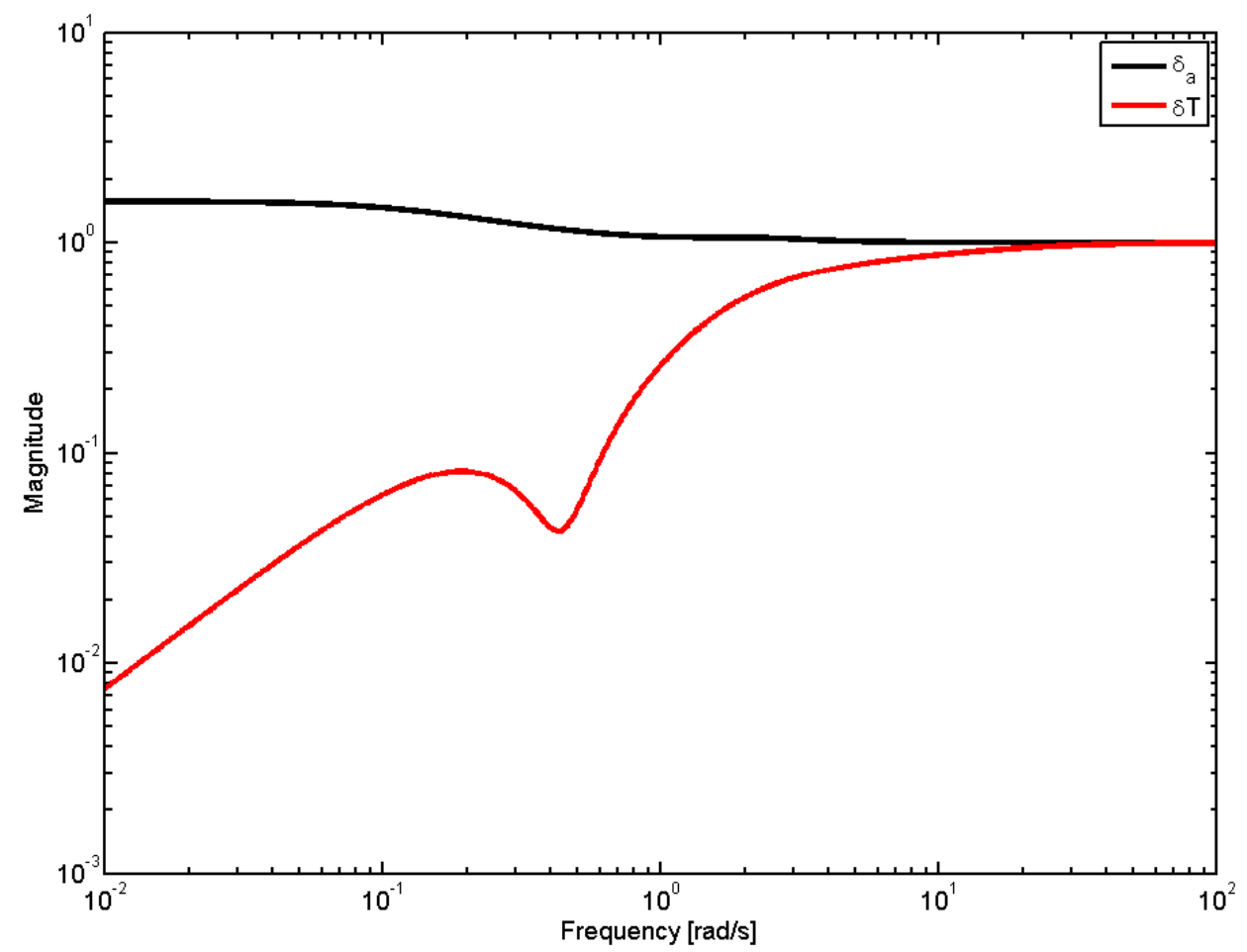

Figure 12.3: Input sensitivity function 


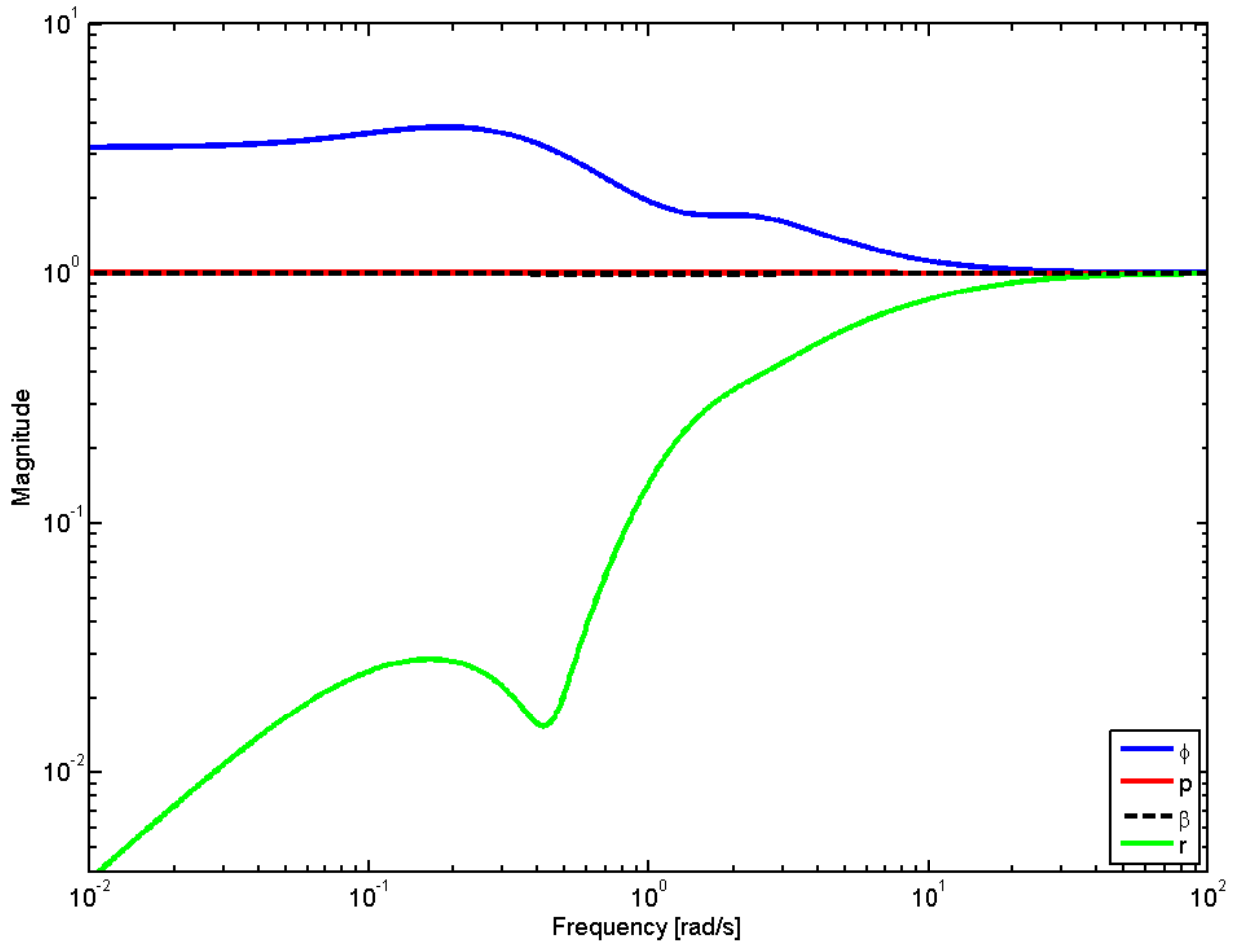

Figure 12.4: Output sensitivity function 
Next, we can investigate the shaped plant behaviors for inputs and outputs in Fig. 12.5 and Fig. 12.6, respectively, where we can see that for the aileron control input $\left(\delta_{a}\right)$, the shaped plant has very good response characteristics of having high gain at low frequencies for tracking and low gain at high frequencies for disturbance rejection. For the differential thrust control input $(\delta T)$, although the gain at low frequencies is not as high as that of the aileron control input, but the gain is relatively linear, which makes the differential thrust control quite predictable for the pilots. At high frequencies, the gain roll-off is also linear and quite similar to that of the aileron control input, which is helpful at rejecting disturbances. In addition, Fig. 12.5 and Fig. 12.6 also show the output responses of the shaped plant in all four lateral/directional states, from which we can see that the implemented controller based on $H_{\infty}$ loop-shaping approach can augment the system in two groups: roll angle and roll rate $(\phi$ and $p)$ and side-slip angle and yaw rate $(\beta$ and $r)$. It is also obvious that roll angle and roll rate $(\phi$ and $p)$ have higher gains than side-slip angle and yaw rate $(\beta$ and $r)$, which is expected when the aircraft loses its vertical stabilizer.

In addition, it is possible to look at the augmented control action by the $H_{\infty}$ loop-shaping controller in Fig. 12.7. Furthermore, the co-sensitivity plots related to the shaped plant behaviors for the inputs and outputs are presented in Fig. 12.8 and Fig. 12.9, respectively. Fig. 12.7, Fig. 12.8, and Fig. 12.9 prove that the controller based on $H_{\infty}$ loop-shaping approach is able to achieve desirable closed loop response characteristics in the frequency domain in terms of robustness and stability. 


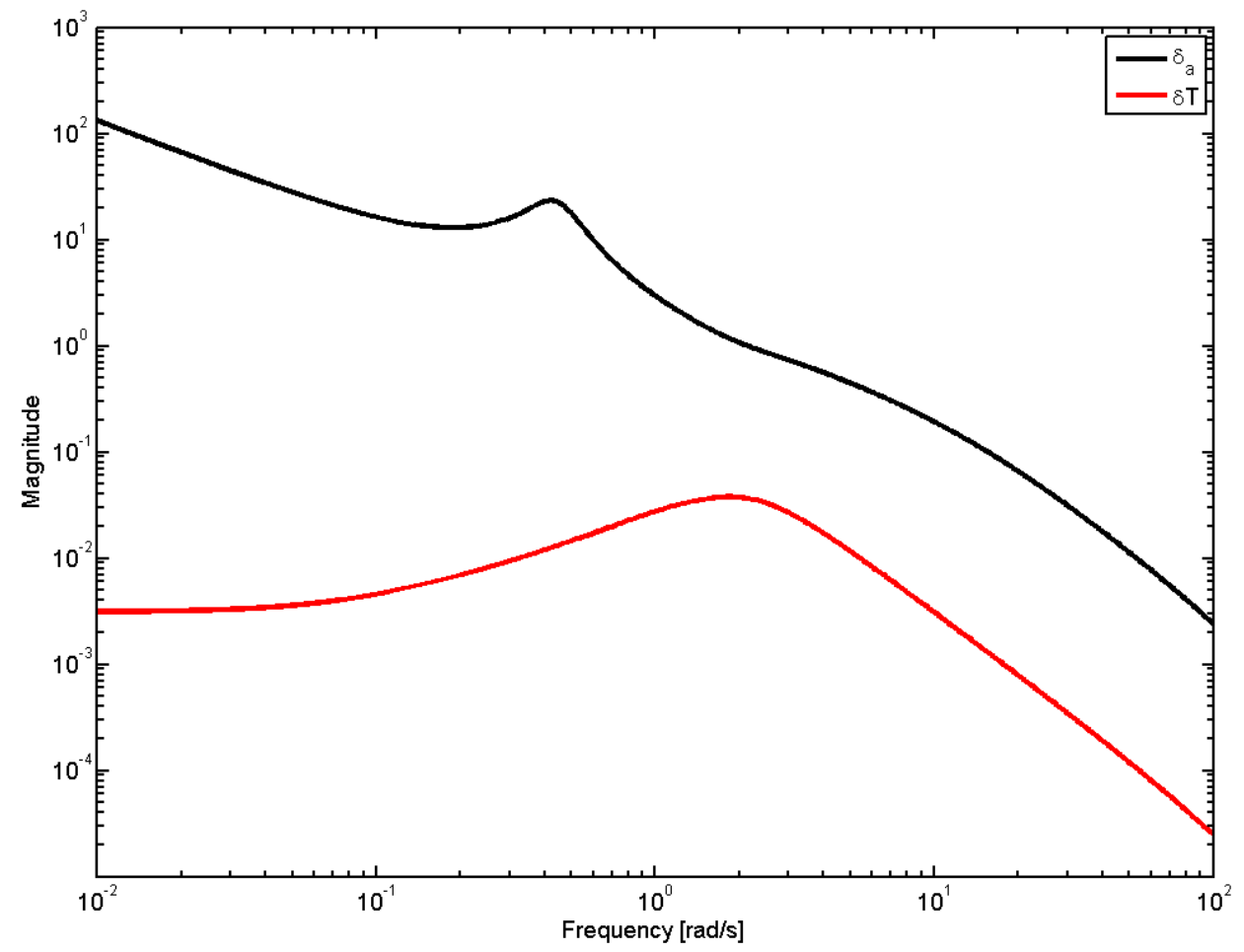

Figure 12.5: Input responses of shaped plant 


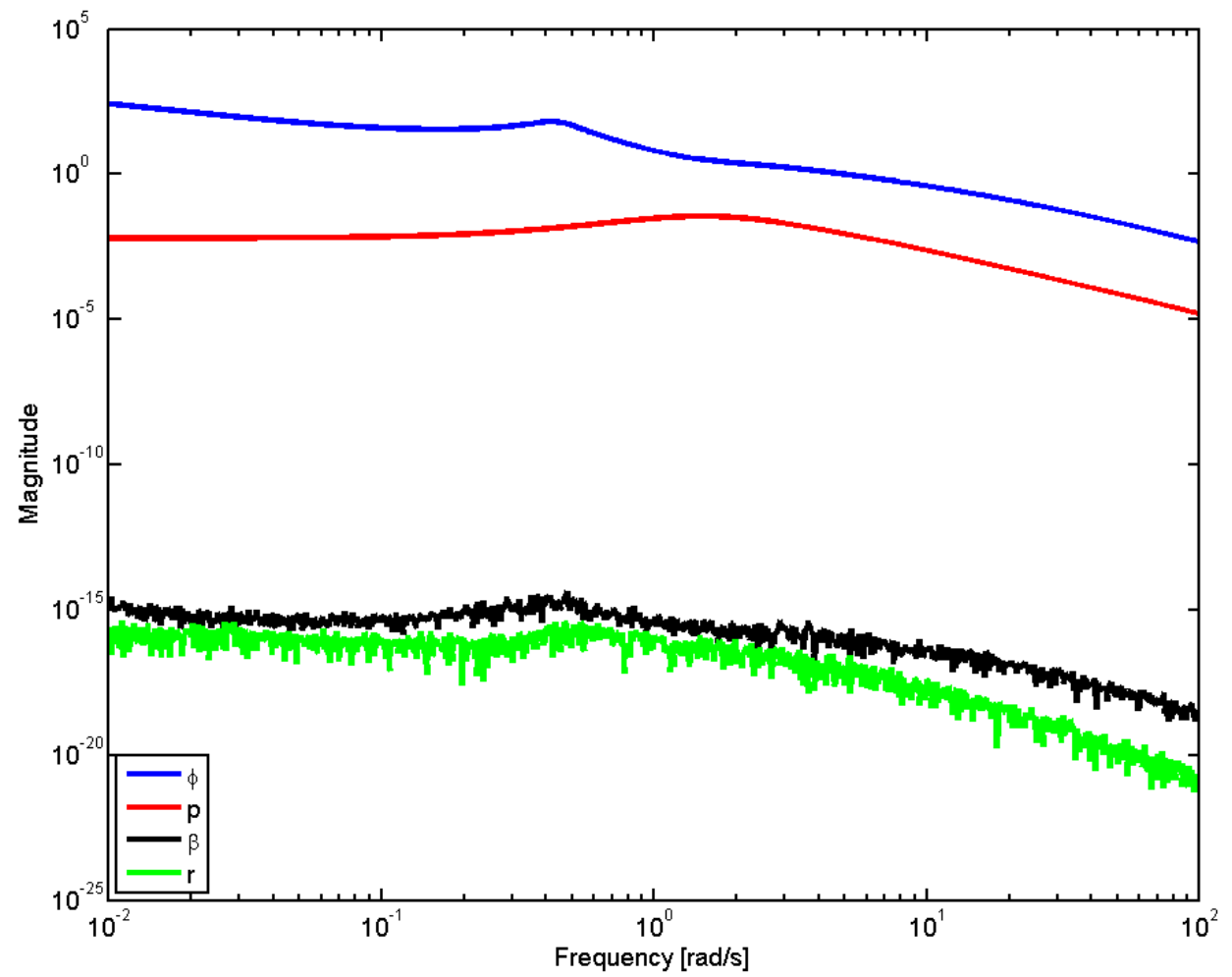

Figure 12.6: Output responses of shaped plant 


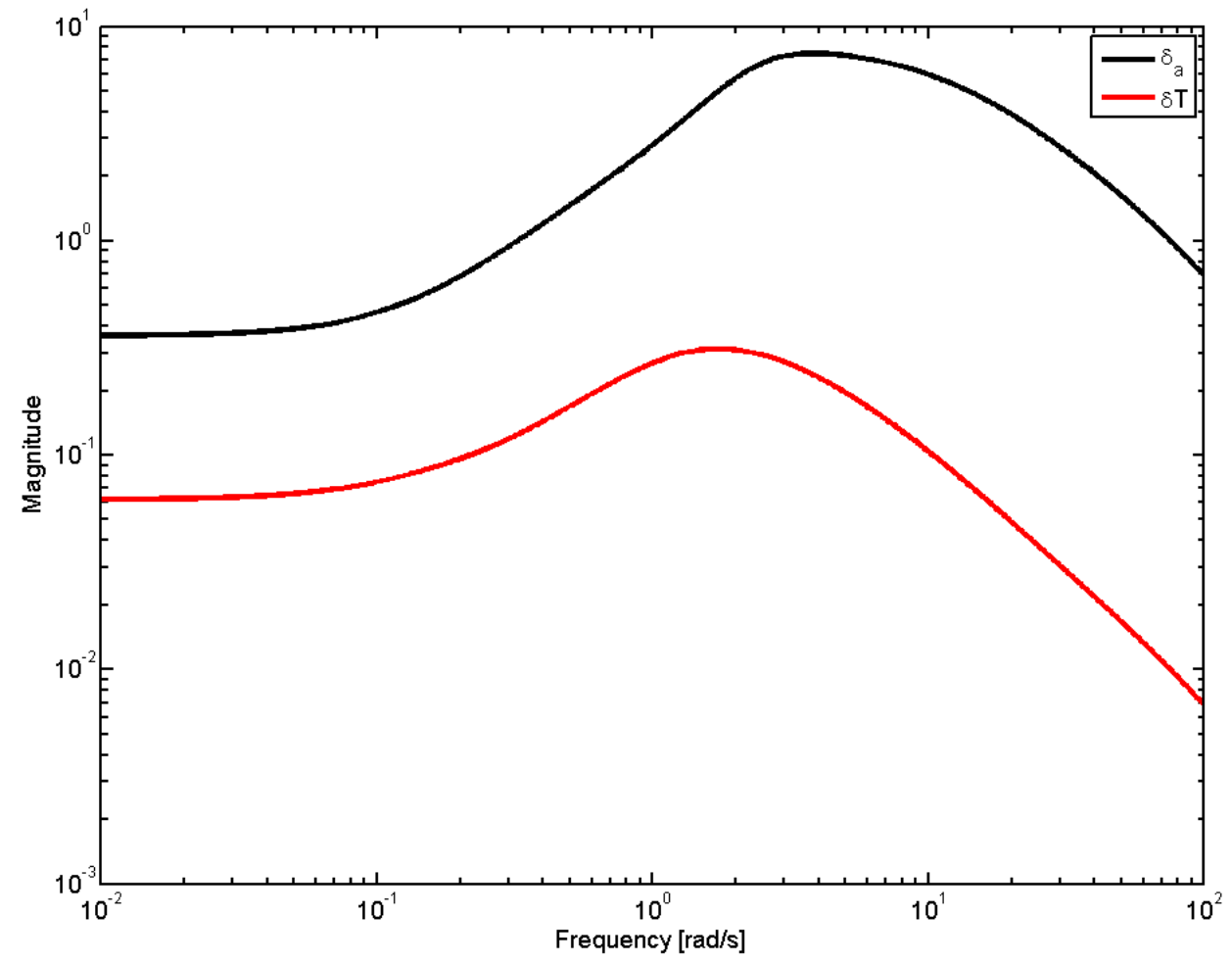

Figure 12.7: Augmented control action by the $H_{\infty}$ loop-shaping robust controller 


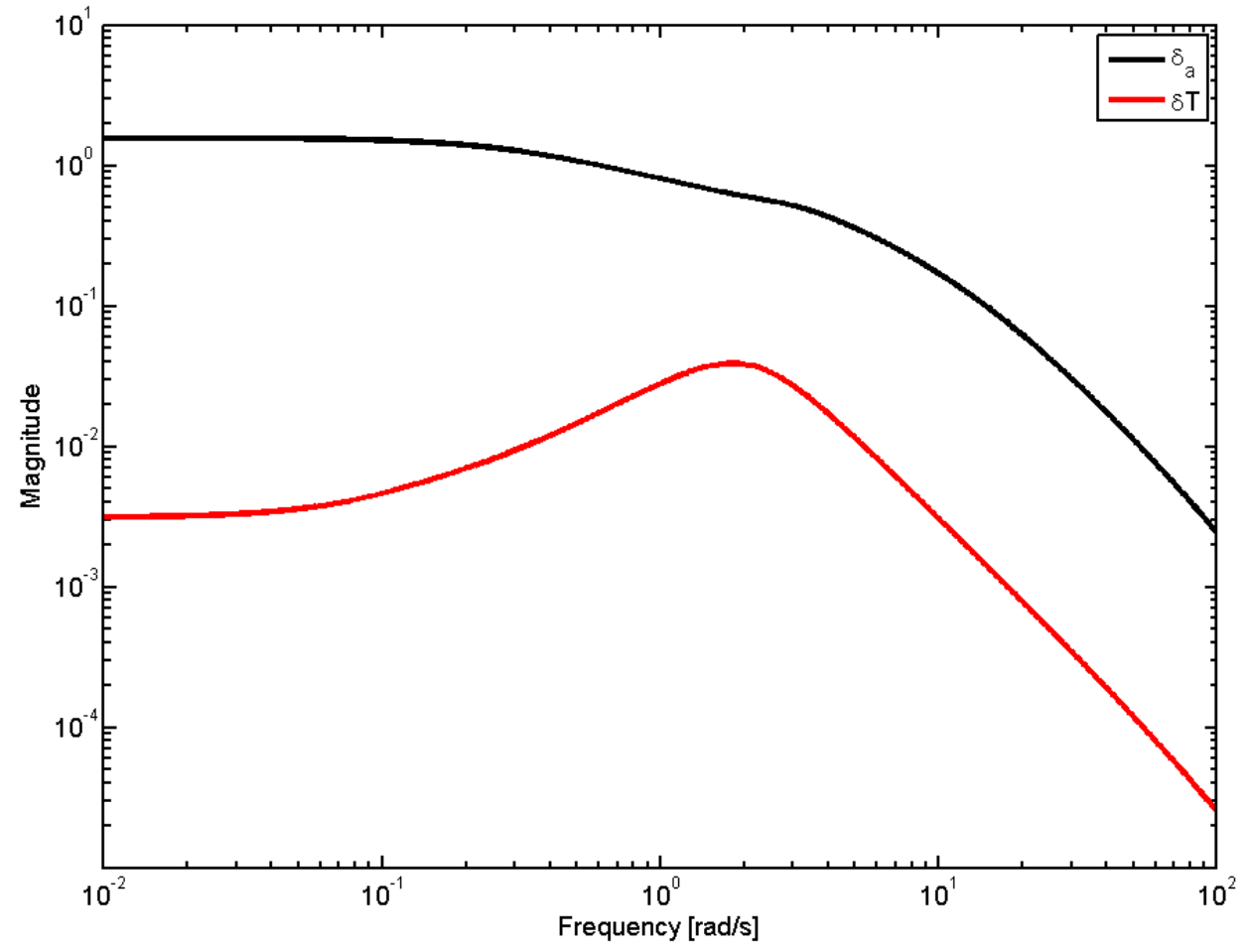

Figure 12.8: Co-sensitivity plot for input responses of shaped plant 


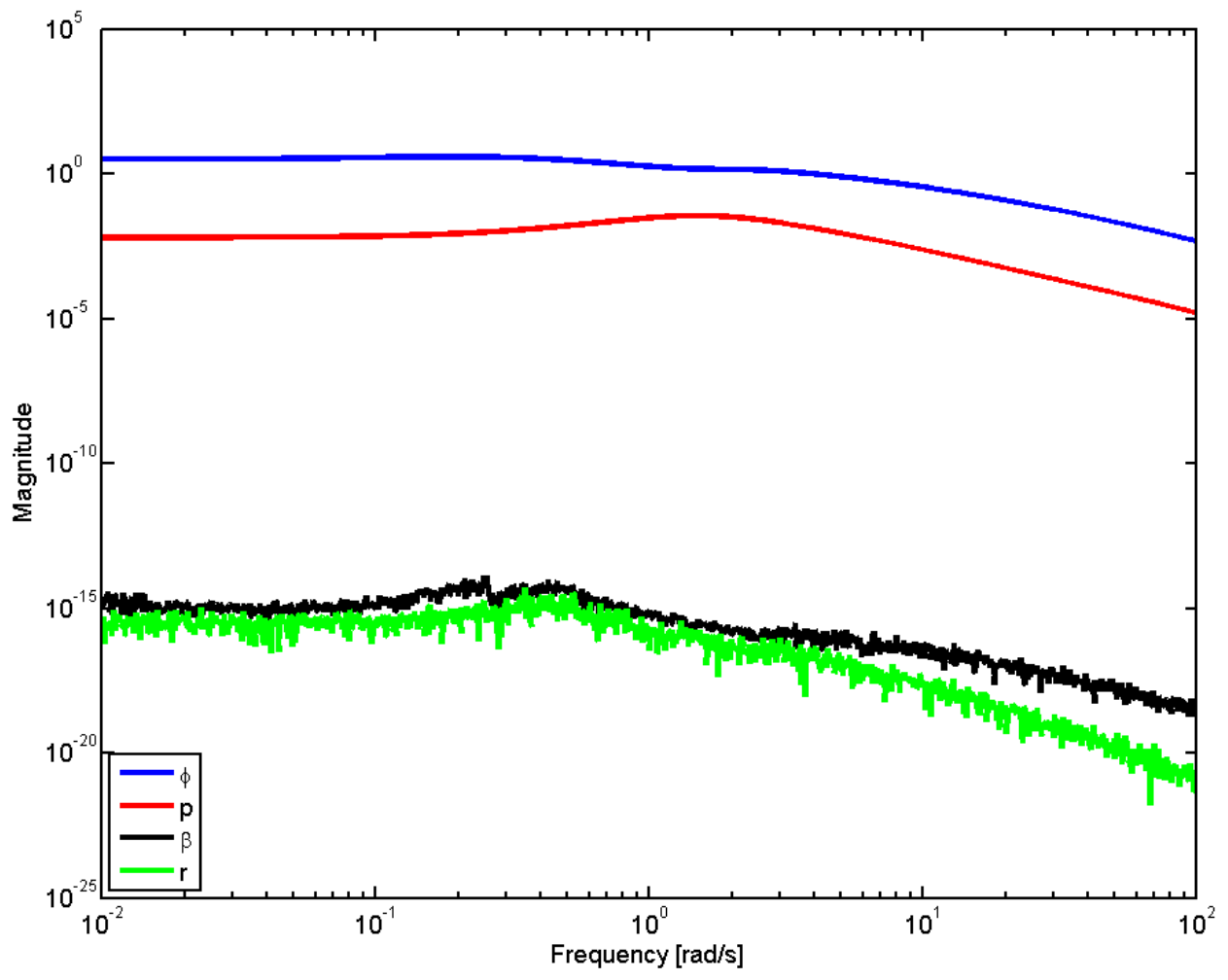

Figure 12.9: Co-sensitivity plot for output responses of shaped plant 
Next, the comparison of the open-loop, shaped, and robustified plant responses is carried out to investigate the effect of the $H_{\infty}$ loop-shaping on the plant dynamics as illustrated in Fig. 12.10 and Fig. 12.11.

As seen in Fig. 12.10 and Fig. 12.11, compared to the open-loop plant responses, the performance of the damaged aircraft is further improved by controller.

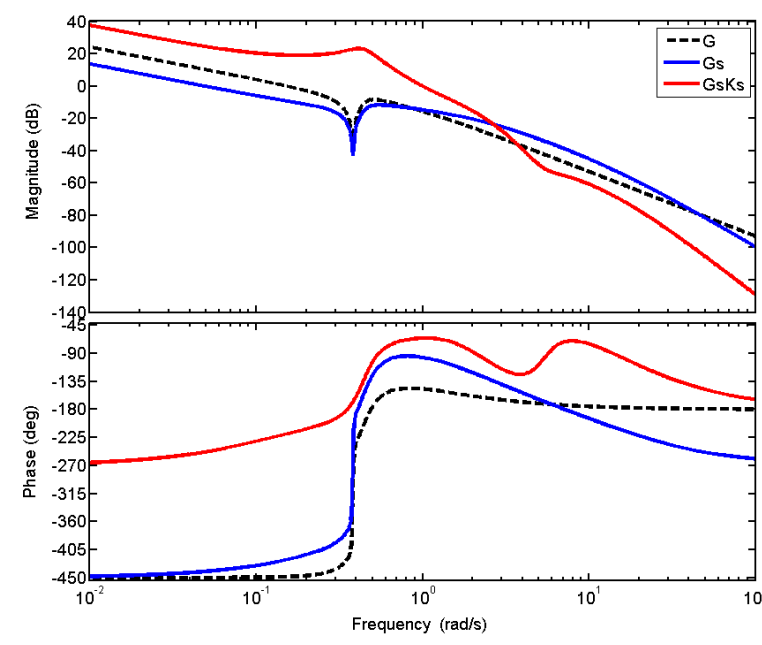

(a) Transfer function $\frac{\phi}{\delta_{a}}$

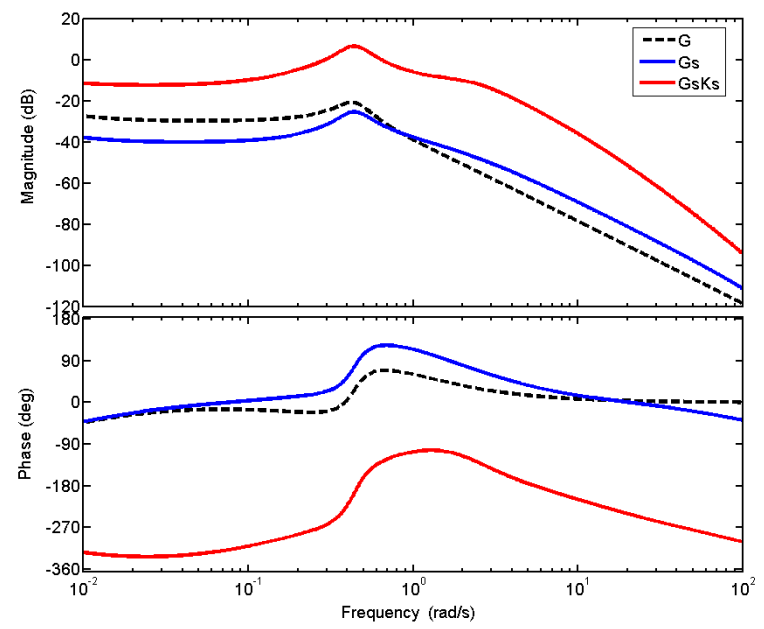

(c) Transfer function $\frac{\beta}{\delta_{a}}$

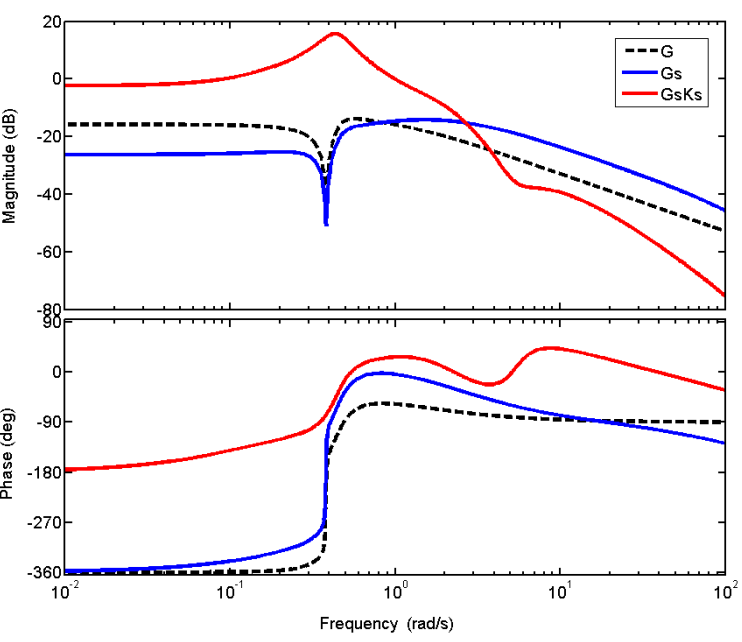

(b) Transfer function $\frac{p}{\delta_{a}}$

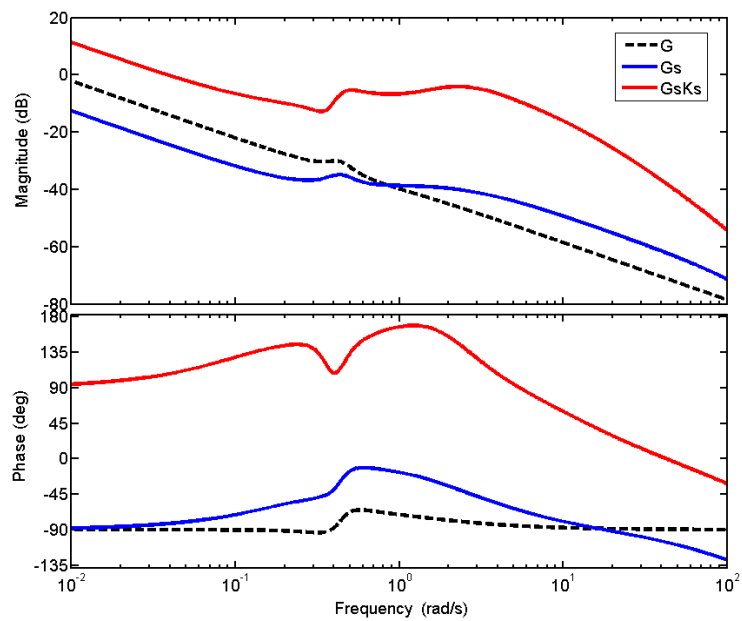

(d) Transfer function $\frac{r}{\delta_{a}}$

Figure 12.10: Open-loop $(G)$ vs. shaped $\left(G_{s}\right)$ vs. robustified $\left(G_{s} K_{s}\right)$ plant for aileron input 


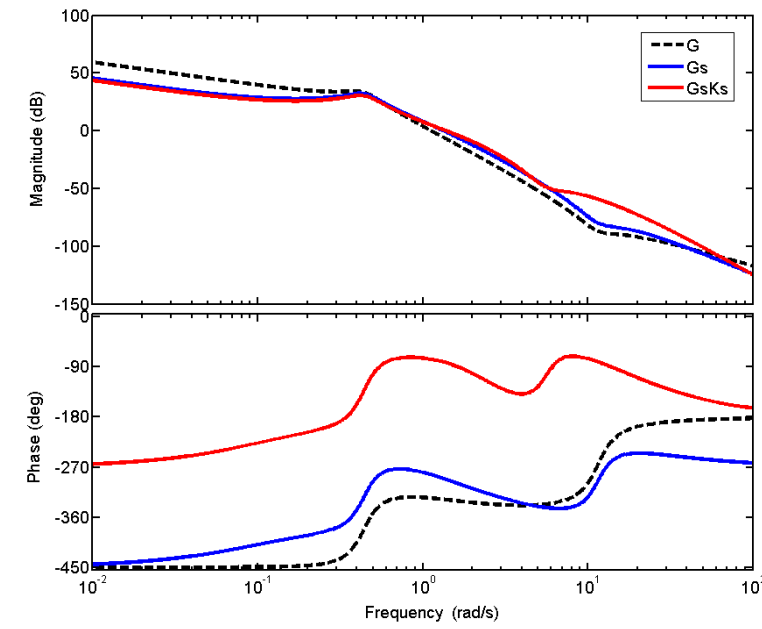

(a) Transfer function $\frac{\phi}{\delta T}$

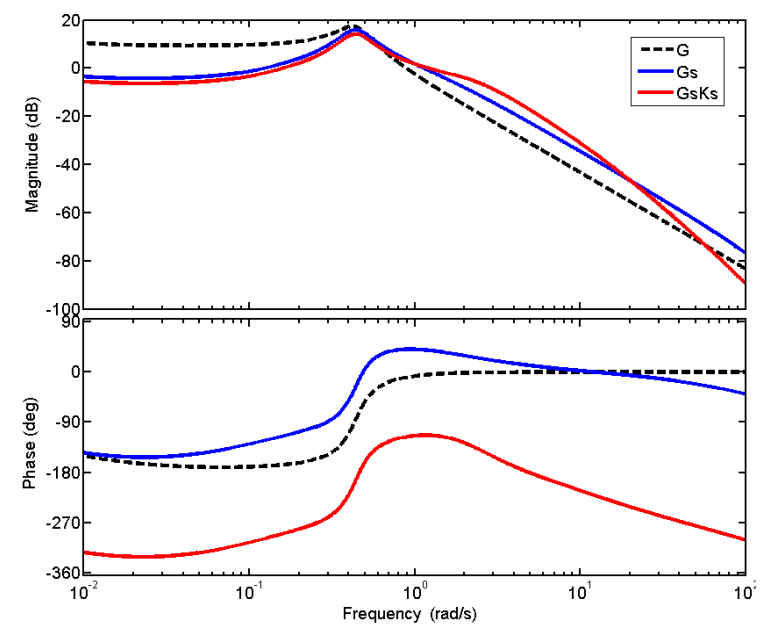

(c) Transfer function $\frac{\beta}{\delta T}$

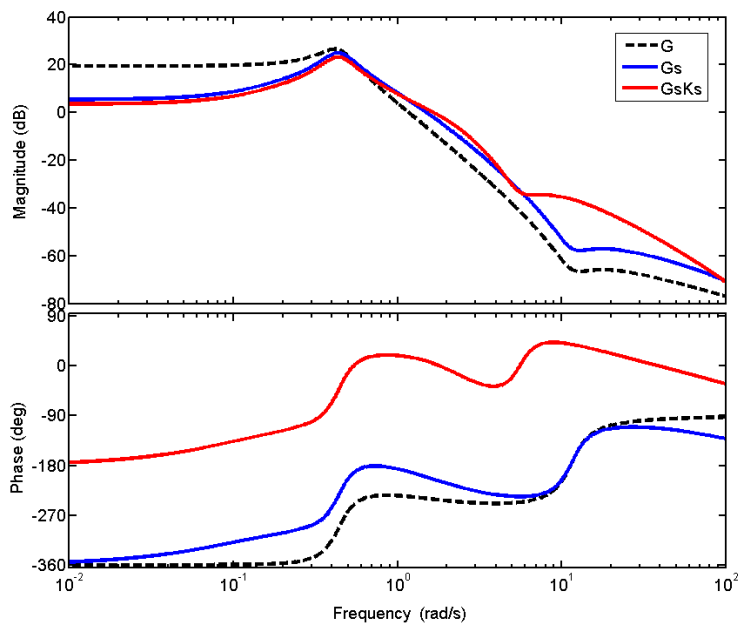

(b) Transfer function $\frac{p}{\delta T}$

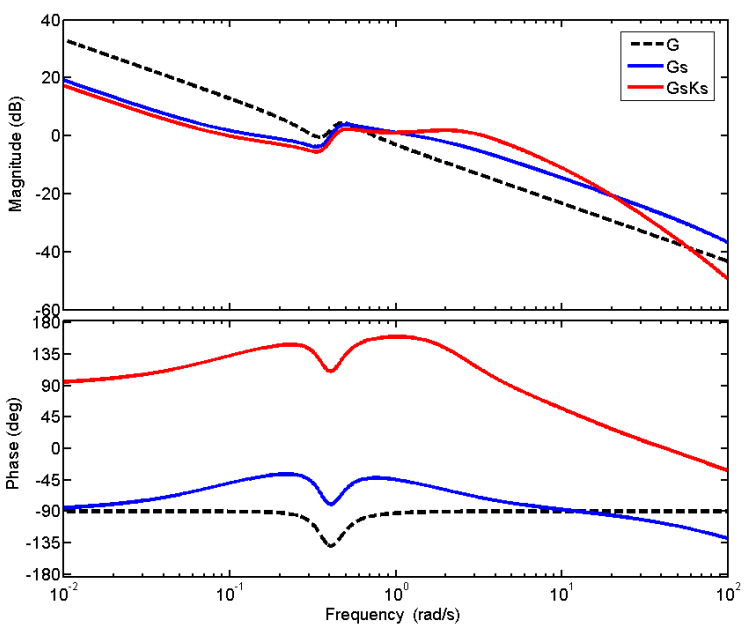

(d) Transfer function $\frac{r}{\delta T}$

Figure 12.11: Open-loop $(G)$ vs. shaped $\left(G_{s}\right)$ vs. robustified $\left(G_{s} K_{s}\right)$ plant for differential thrust input 
Furthermore, it is also worth checking the allowable gain and phase margin variations (i.e. associated uncertainty balls in gain and phase margins for a robust response and guaranteed stability). Obtained results for the disk margins for the inputs and outputs are described in Fig. 12.12 and Fig. 12.13, respectively. From Fig. 12.12 and Fig. 12.13, it can be clearly seen that with the $H_{\infty}$ loop-shaping control system design, both the inputs and outputs can achieve desirable and safe stability margins. For the inputs, the maximum phase margin is approximately 78 degrees while the maximum gain margin is about $19 \mathrm{~dB}$. For the outputs, the maximum phase margin is approximately 36 degrees while the maximum gain margin is about $5.8 \mathrm{~dB}$.

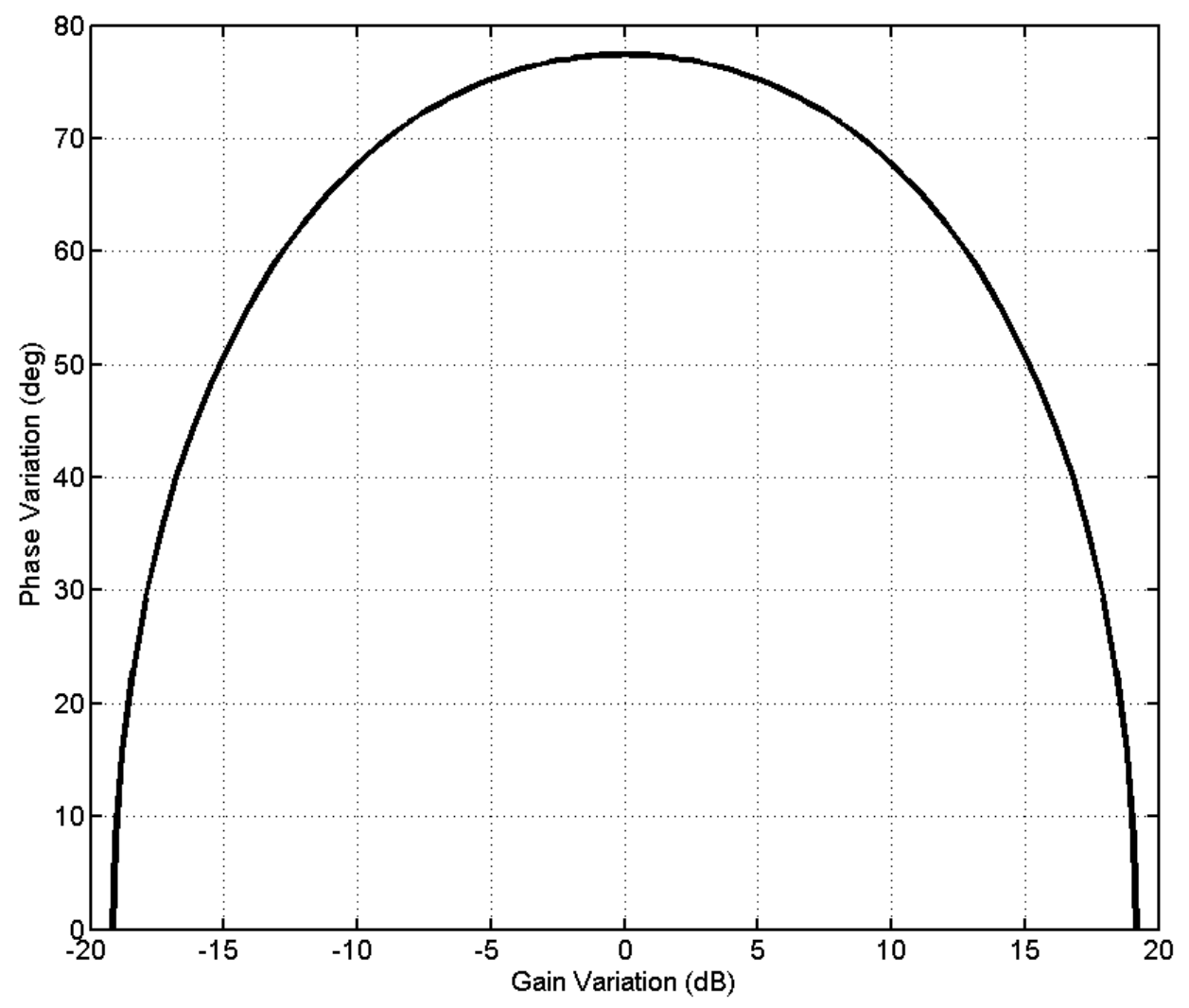

Figure 12.12: Disk margins for inputs 


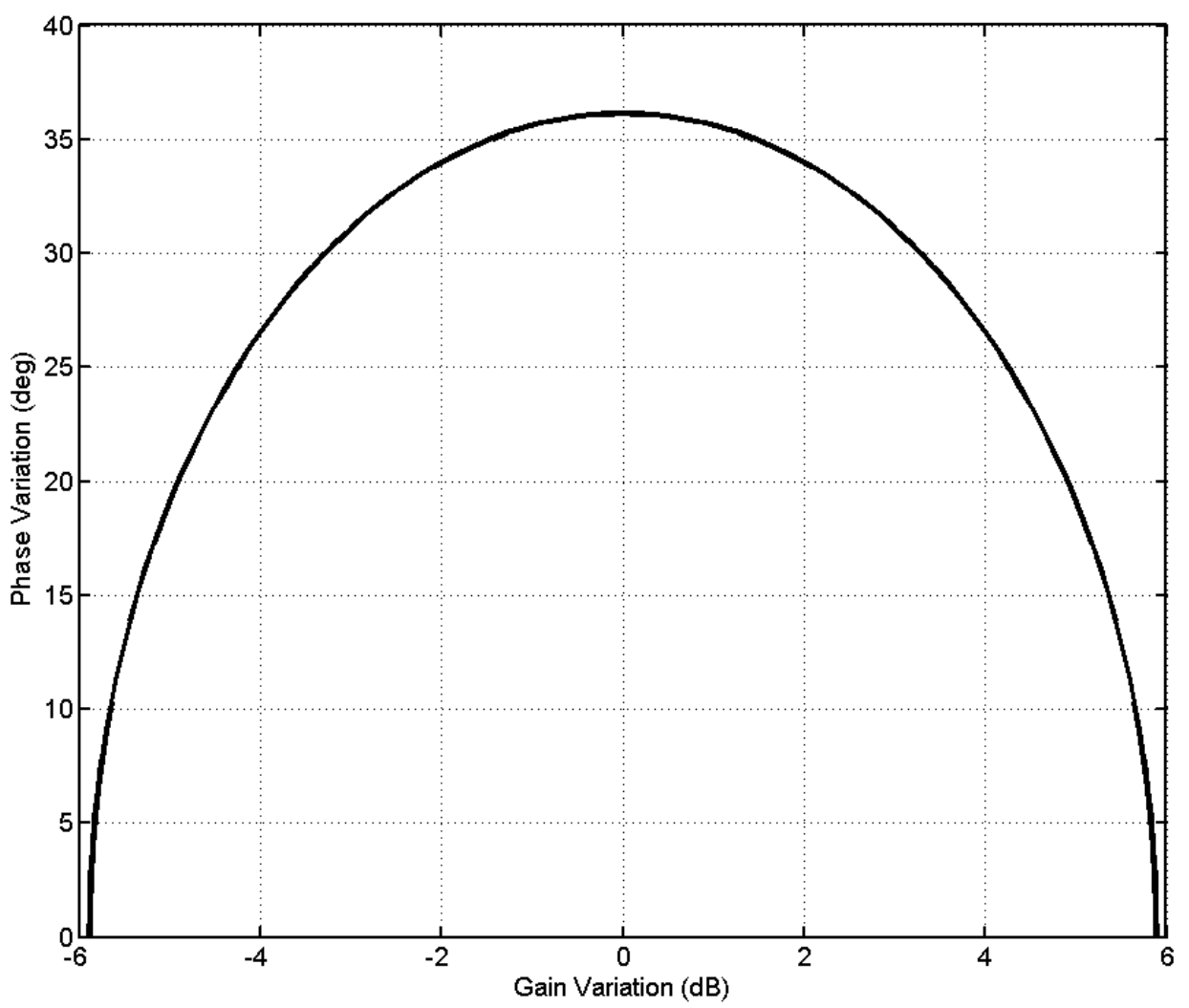

Figure 12.13: Disk margins for outputs 


\subsection{Simulation Results}

Now it is time to look at the time domain responses of the damaged aircraft, which are shown in Fig. 12.14 and Fig. 12.15. It is worth mentioning that the control inputs for both plants are one degree step inputs for both the ailerons and differential thrust to simulate an extreme scenario test to see whether the damaged aircraft utilizing differential thrust can hold itself in a continuous yawing and banking maneuver without becoming unstable and losing control. From Fig. 12.14, it is obvious that after only 15 seconds, all four states of the aircraft's lateral/directional dynamics reach steady state values, which means that the controller can stabilize the damaged aircraft in only 15 seconds. It is also worth noticing that with the help of differential thrust acting as a "virtual rudder" input during the coordinated turn maneuver, the aircraft was able to achieve zero side-slip angle. Additionally, as seen in Fig. 12.15, the heading angle gain becomes very linear after 15 seconds, and for one degree step inputs, the heading angle gain is about 3.6 degrees after 30 seconds. However, this comes at the cost of the control efforts as shown in Fig. 12.16, which are still under the control limits and without any saturation of the actuators.

As discussed previously, in order to have a feasible control strategy in real-life situation, limiting factors are imposed on the aileron and differential thrust control efforts. The aileron deflection is limited at \pm 26 degrees [18]. For differential thrust, a differential thrust saturation value is set at $43,729 \mathrm{lbf}$, which is the difference of the maximum thrust and trimmed thrust values of the JT9D-7A engine. In addition, a rate limiter is also imposed on the thrust response characteristic at 12,726 lbf/s as discussed in Chapter 7.

The aileron control effort, as indicated by Fig. 12.16, calls for the maximum 

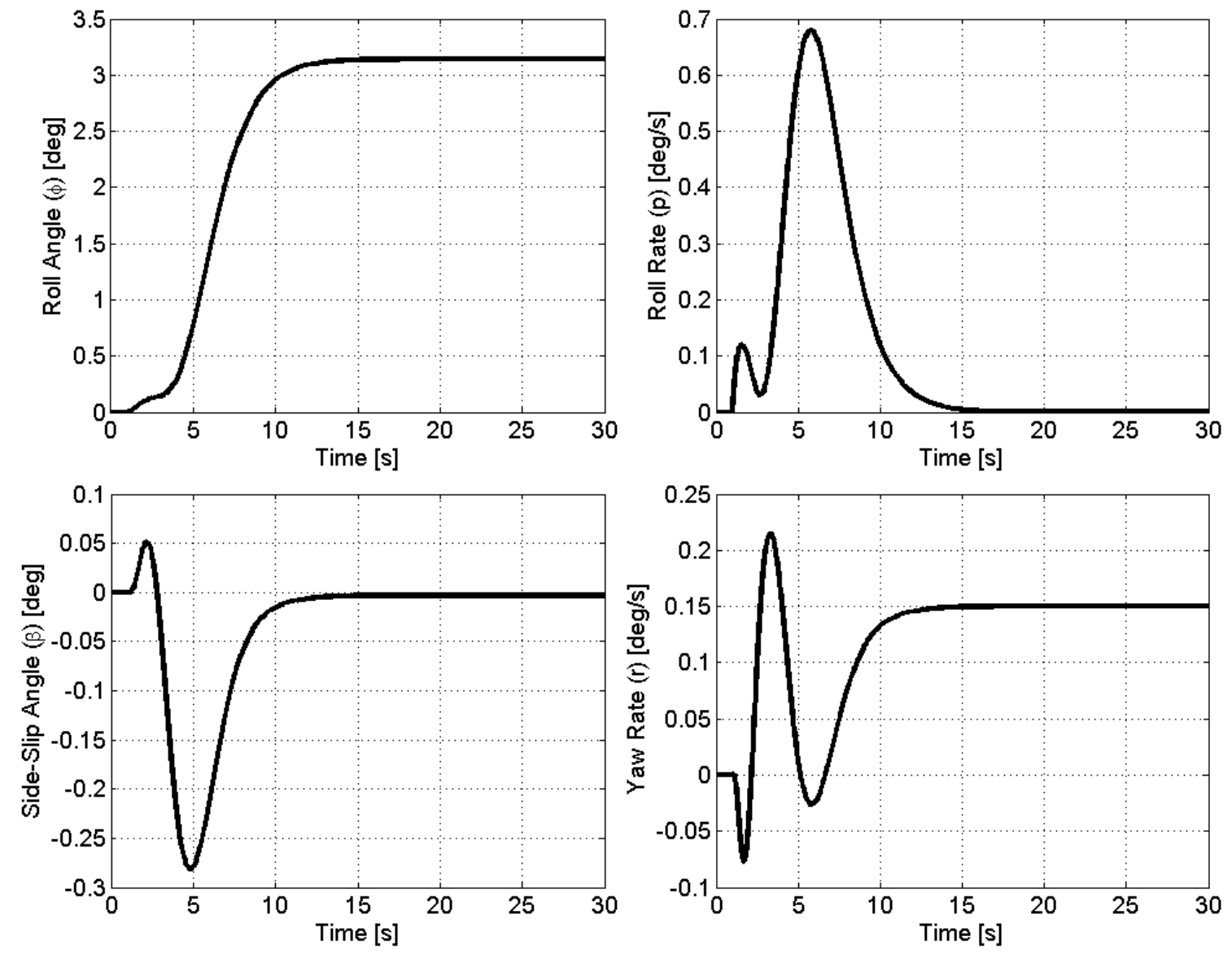

Figure 12.14: Closed loop responses of the damaged aircraft with a robust controller 


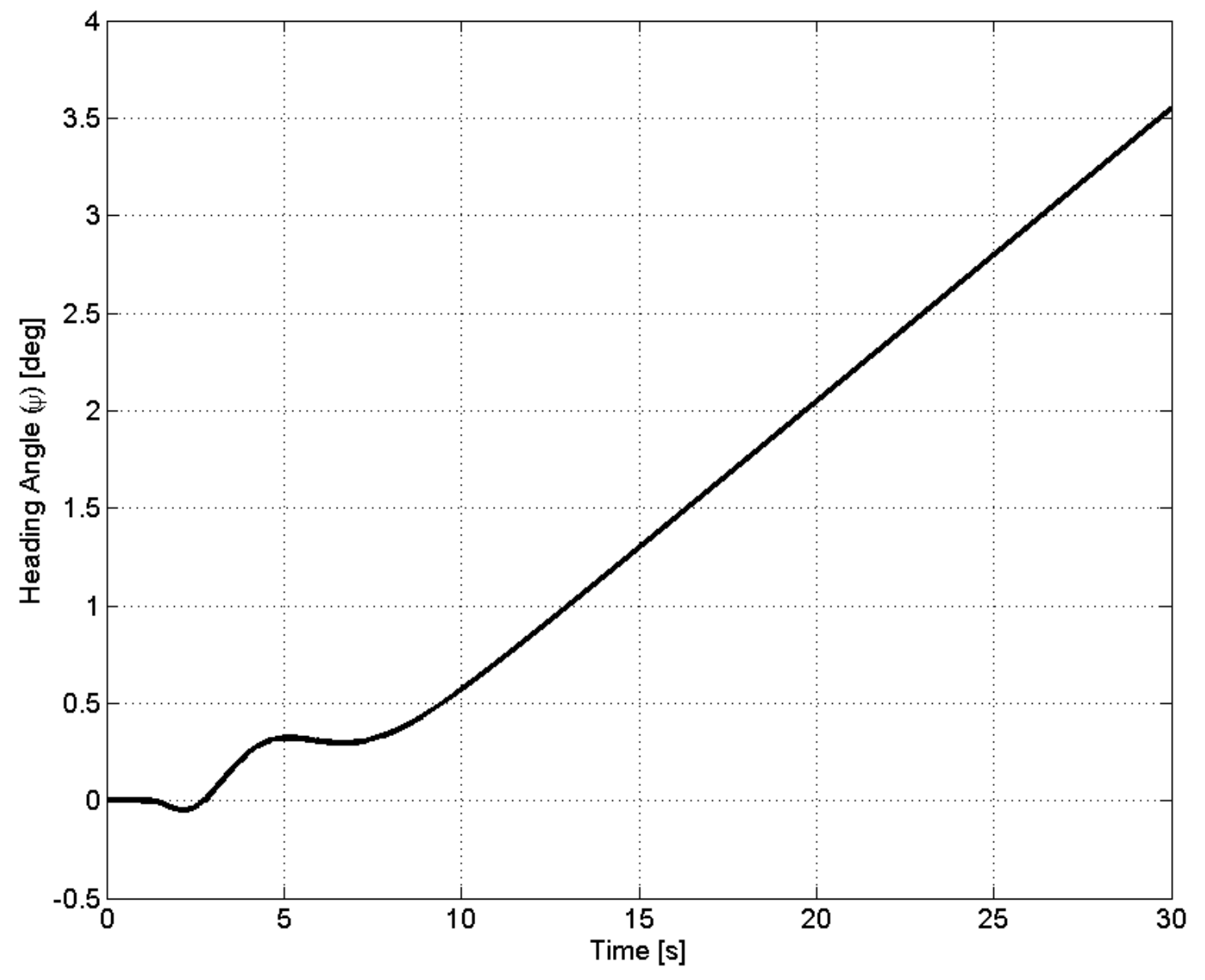

Figure 12.15: Closed loop heading angle response with a robust controller 

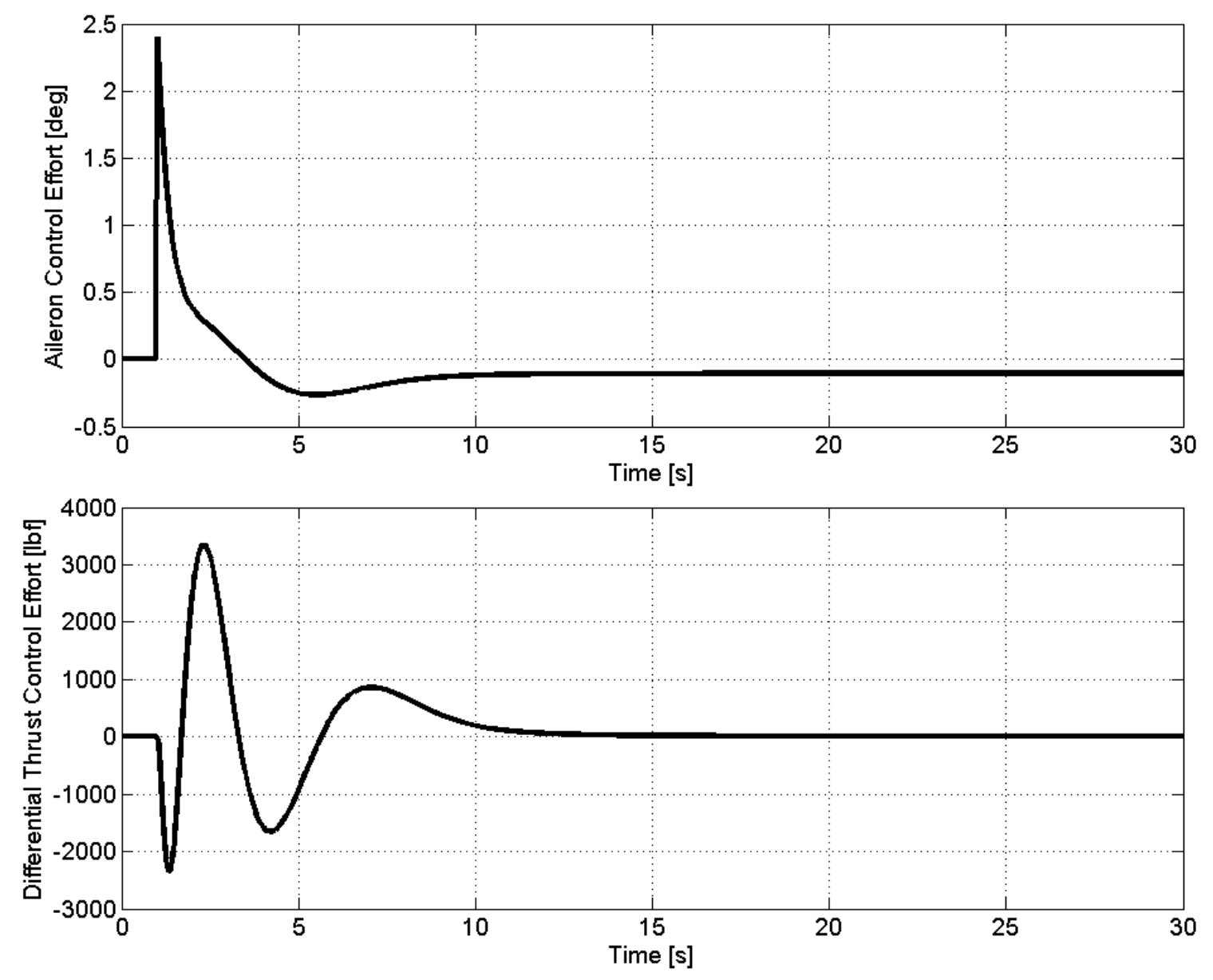

Figure 12.16: Closed loop control efforts with a robust controller 
deflection of approximately 2.4 degrees and reaches steady state at approximately -0.1 degree of deflection after 15 seconds. This aileron control effort is very reasonable and achievable if the ailerons are assumed to have instantaneous response characteristics by neglecting the lag from actuators or hydraulic systems. The differential thrust control effort demands a maximum differential thrust of approximately $3350 \mathrm{lbf}$, which is well within the thrust capability of the JT9D-7A engine, and the differential thrust control effort reaches steady state at around 15 lbf after 15 seconds. Therefore, it can be concluded that the robust control system design based on the $H_{\infty}$ loop-shaping approach is proven to be able to stabilize and save the damaged aircraft.

\subsection{Robustness and Sensitivity Analysis of $H_{\infty}$ Loop-Shaping Based Robust Control System}

The robustness and sensitivity of the $H_{\infty}$ loop-shaping based robust control system are examined by the introduction of band-limited white noise and additive uncertainty parameters associated with the state matrix of the damaged aircraft to investigate the performance of the robust control system in the presence of noise and uncertainty.

\subsubsection{Noise Sensitivity Analysis of $H_{\infty}$ Loop-Shaping Based Robust Control System}

The noise sensitivity of the $H_{\infty}$ loop-shaping based robust control system presented in this thesis is investigated by introducing band-limited white noise to test the performance of the robust control system in the presence of noise. Fig. 12.17 shows the logic behind the noise sensitivity analysis of the robust control system. 


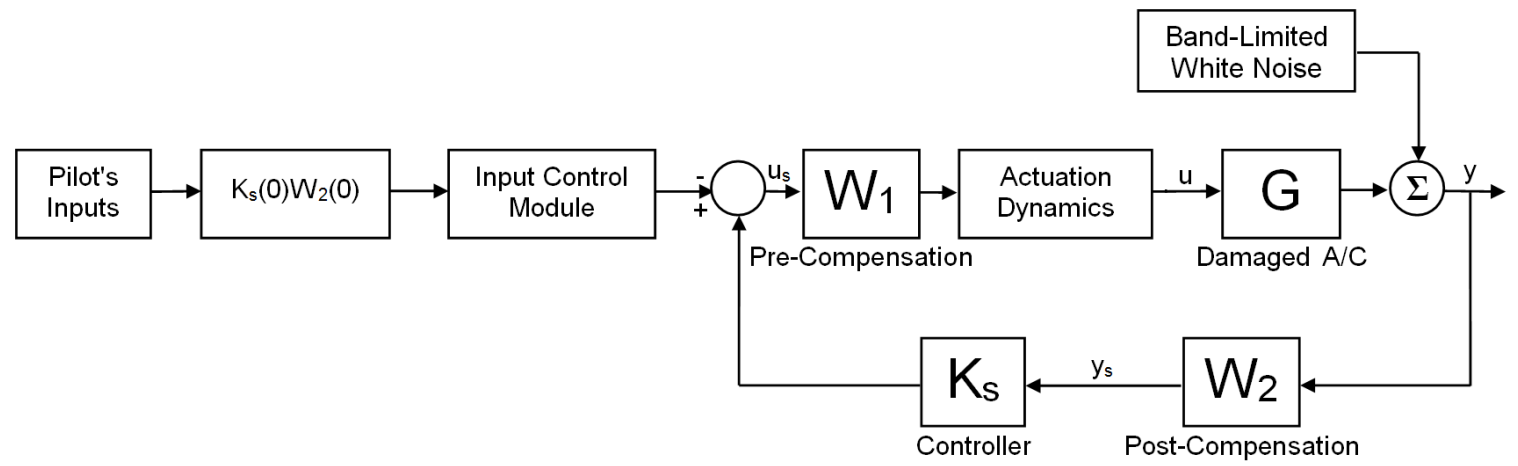

Figure 12.17: Block diagram for $H_{\infty}$ loop-shaping based robust control system design in the presence of noise 
The noise power is set at $10^{-8}$ with sampling time of 0.1 second. The band-limited white noise for the $H_{\infty}$ loop-shaping based robust control system is depicted in Fig. 12.18, and its periodogram using Fast Fourier Transform (FFT) is shown in Fig. 12.19.

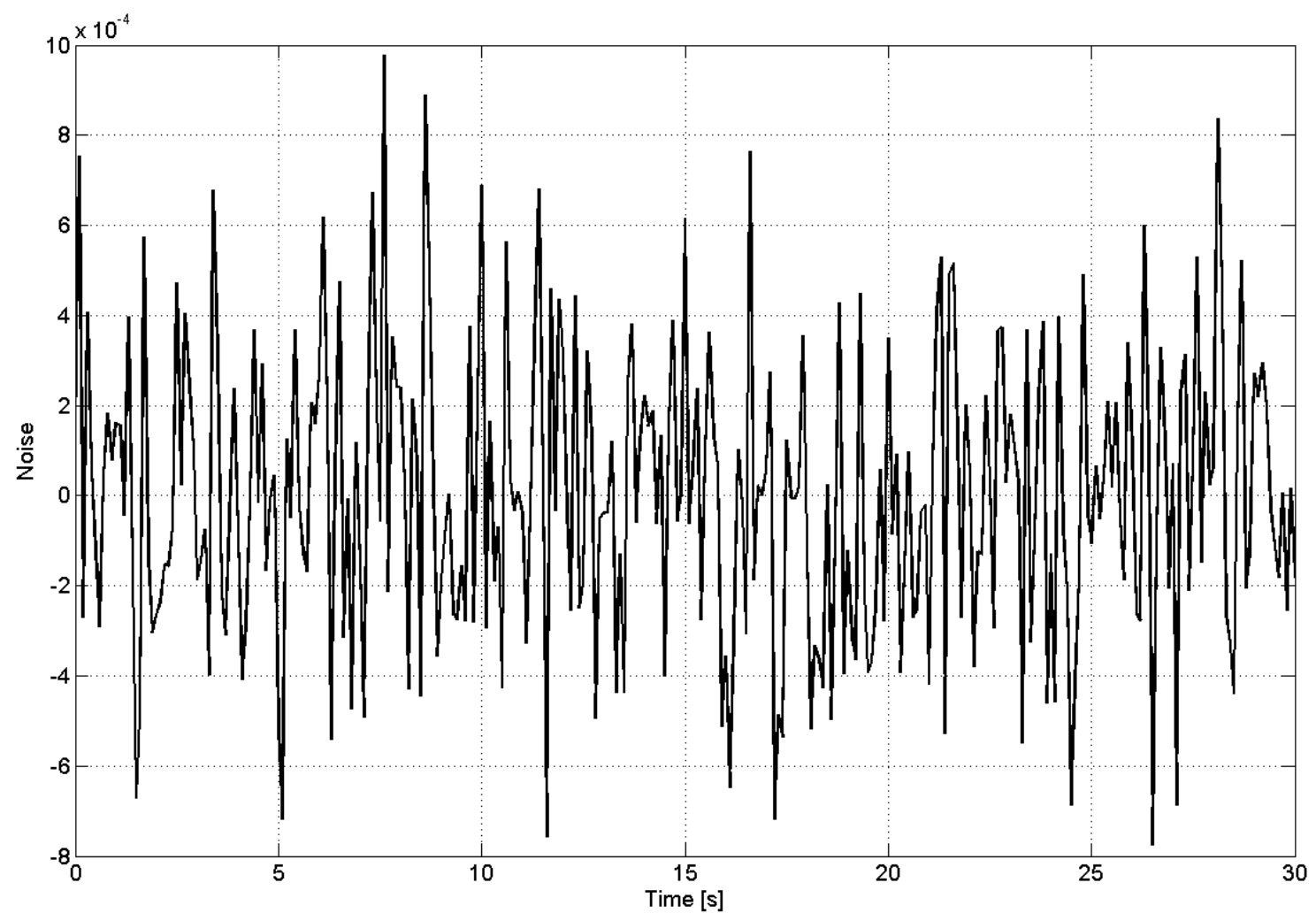

Figure 12.18: Band-limited white noise

Additionally, the closed loop responses of the damaged aircraft with an robust controller in the presence of band-limited white noise are shown in Fig. 12.20 and Fig. 12.21.

From Fig. 12.20, it is obvious that the robust control system design is able to stabilize the damaged aircraft within approximately 15 seconds in the presence of noise. We can also see that all four state outputs are virtually unaffected by the band-limited white noise, which clearly demonstrates the robustness of the $H_{\infty}$ 


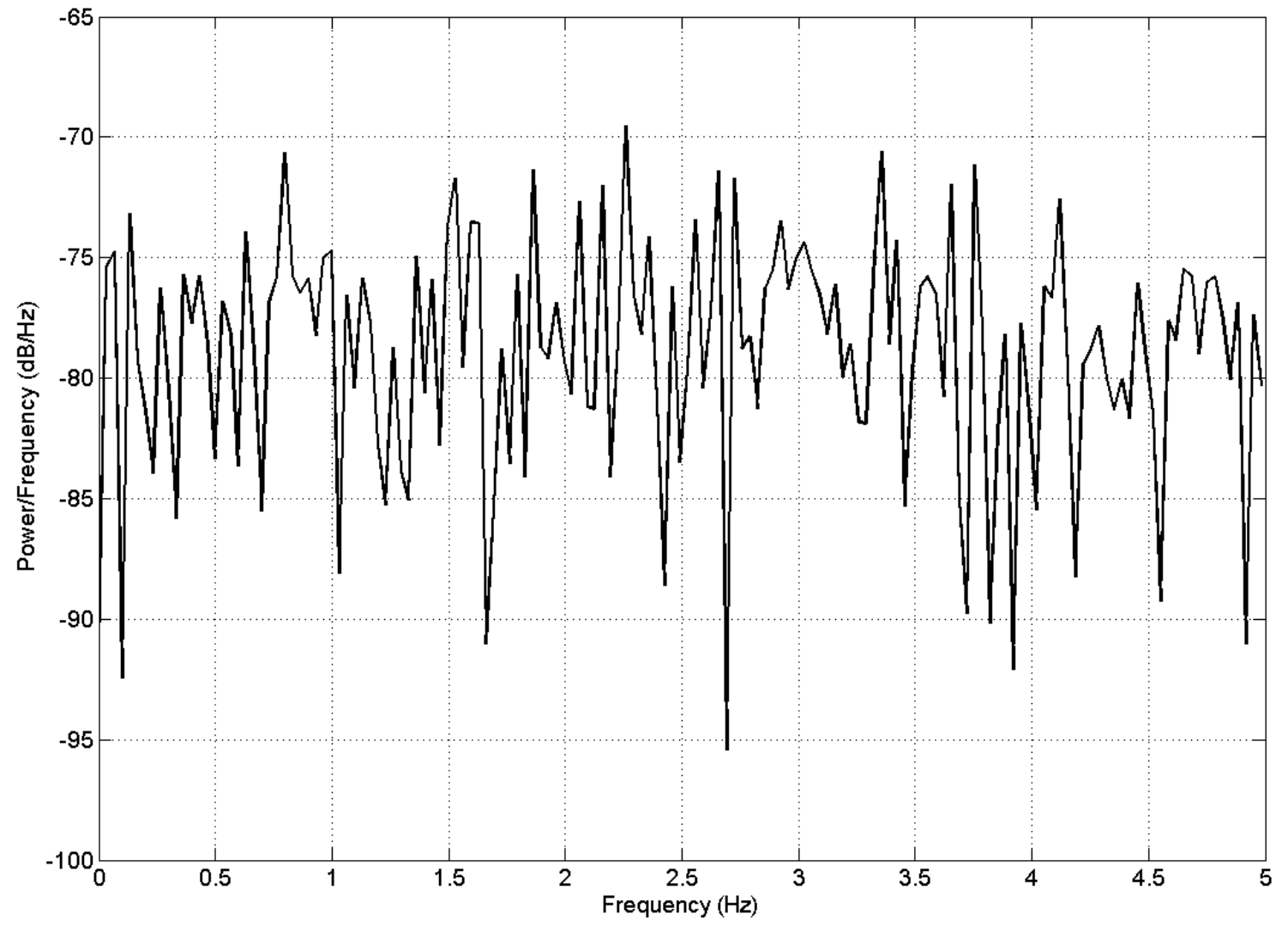

Figure 12.19: Periodogram using Fast Fourier Transform 

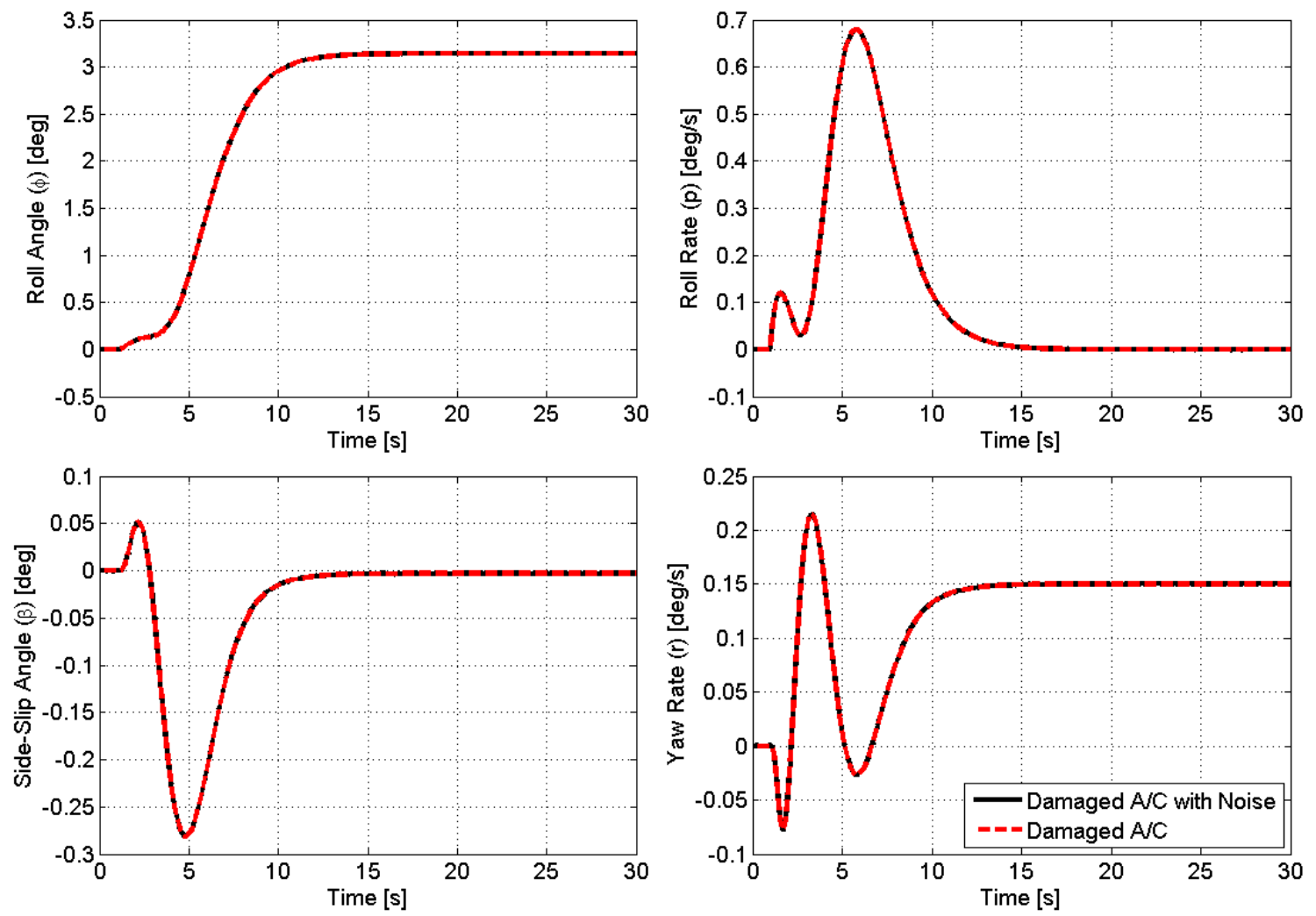

Figure 12.20: Closed loop responses of the damaged aircraft with a robust controller in the presence of noise 


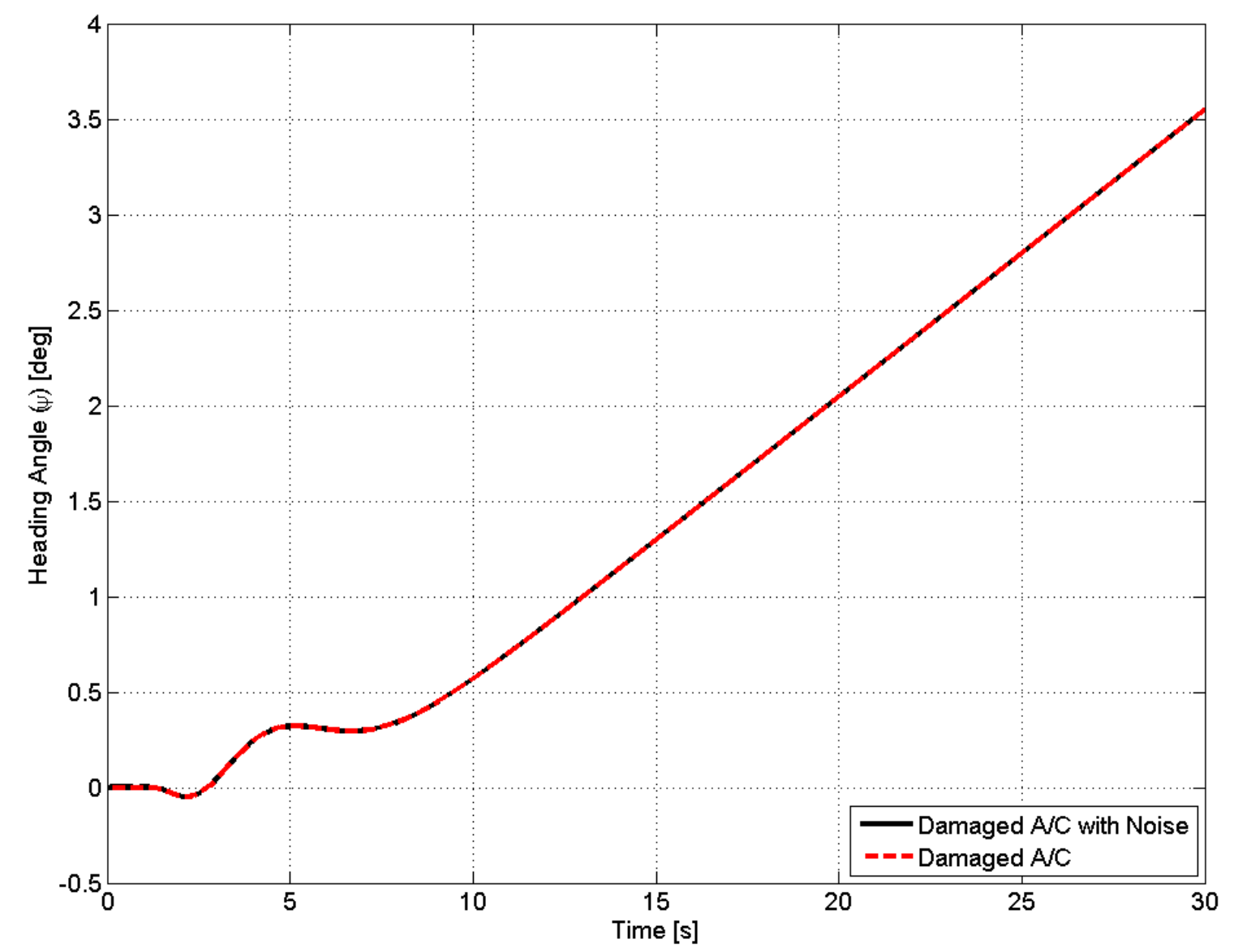

Figure 12.21: Closed loop heading angle response with a robust controller in the presence of noise 
loop-shaping based robust control system.

As for the heading angle response $(\psi)$ shown in Fig. 12.21, the effect of noise is attenuated by the integral action (because the heading angle $(\psi)$ is the integral of the yaw rate $(r)$ ), and the heading angle gain stays the same as when there is no noise: for one degree step inputs, the heading angle gain is about 3.6 degrees within 30 seconds.

Additionally, it is also worth investigating the control efforts required in the presence of noise as seen in Fig. 12.22.
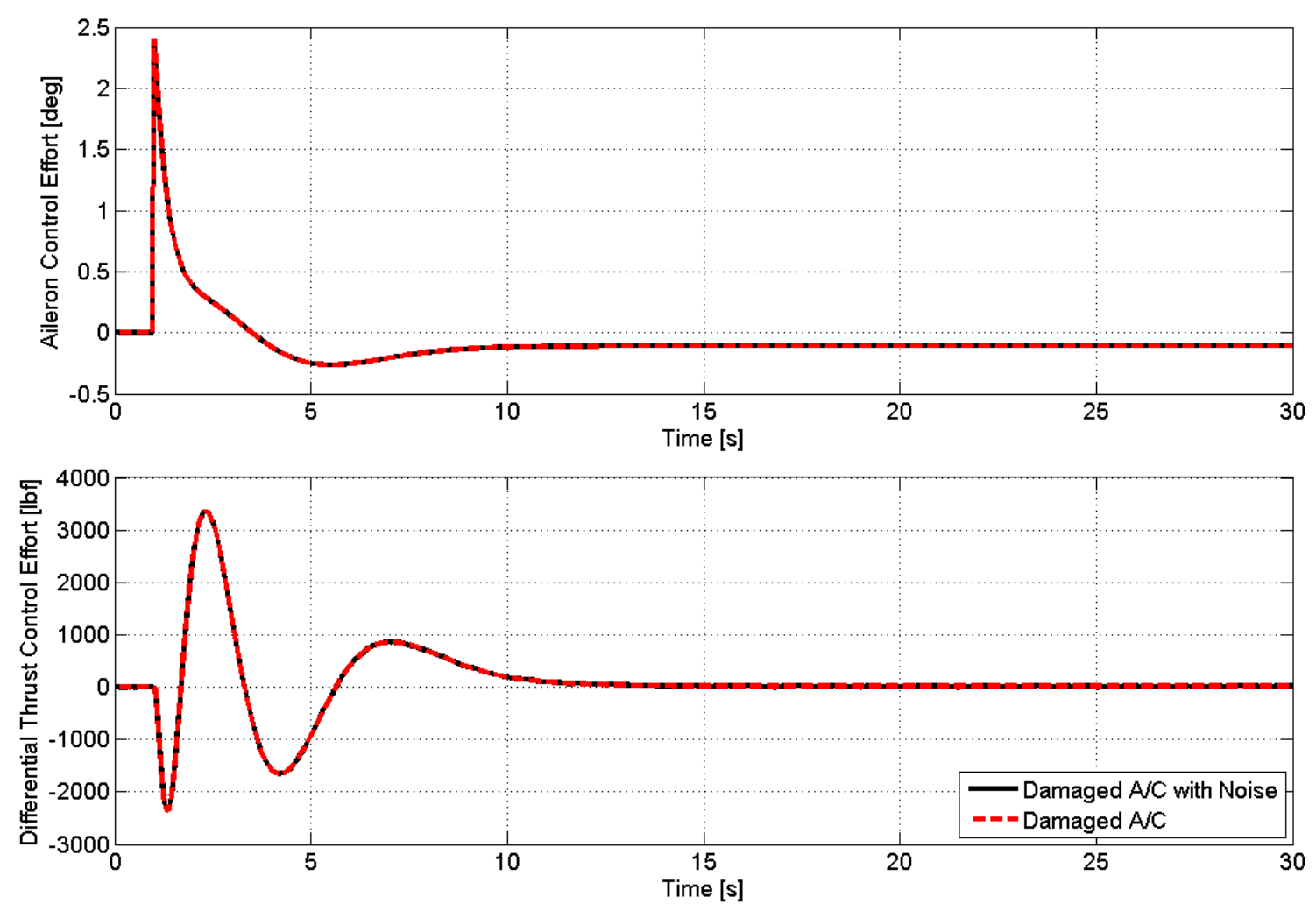

Figure 12.22: Closed loop control efforts with a robust controller in the presence of noise

According to Fig. 12.22, in the presence of noise, the aileron control effort stays virtually unchanged, which still demands the maximum deflection of 
approximately 2.4 degrees and reaches steady state at around -0.1 degree as when there is no noise. This aileron control effort demands are reasonable and feasible due to the limiting factors of \pm 26 degrees of the aileron deflection [18] and the assumption that ailerons have instantaneous response characteristics by neglecting the lag from actuators or hydraulic systems. The differential thrust control effort also stays unchanged, and it still demands at maximum approximately $3350 \mathrm{lbf}$ and reaches steady state value of 15 lbf. Again, due to the differential thrust saturation value set at 43,729 $\mathrm{lbf}$ and the thrust rate limiter set at $12,726 \mathrm{lbf} / \mathrm{s}$, this differential thrust control effort in the presence of noise is feasible in a real life situation.

\subsubsection{Robustness and Uncertainty Analysis of $H_{\infty}$ Loop-Shaping Based Robust Control System}

The robustness of the $H_{\infty}$ loop-shaping based robust differential thrust control system design presented in this thesis is investigated by the introduction of $30 \%$ of full block, additive uncertainty into the plant dynamics of the damaged aircraft to test the performance of the aircraft in the presence of uncertainty. Fig. 12.23 shows the logic behind the robust control system design in the presence of uncertainty.

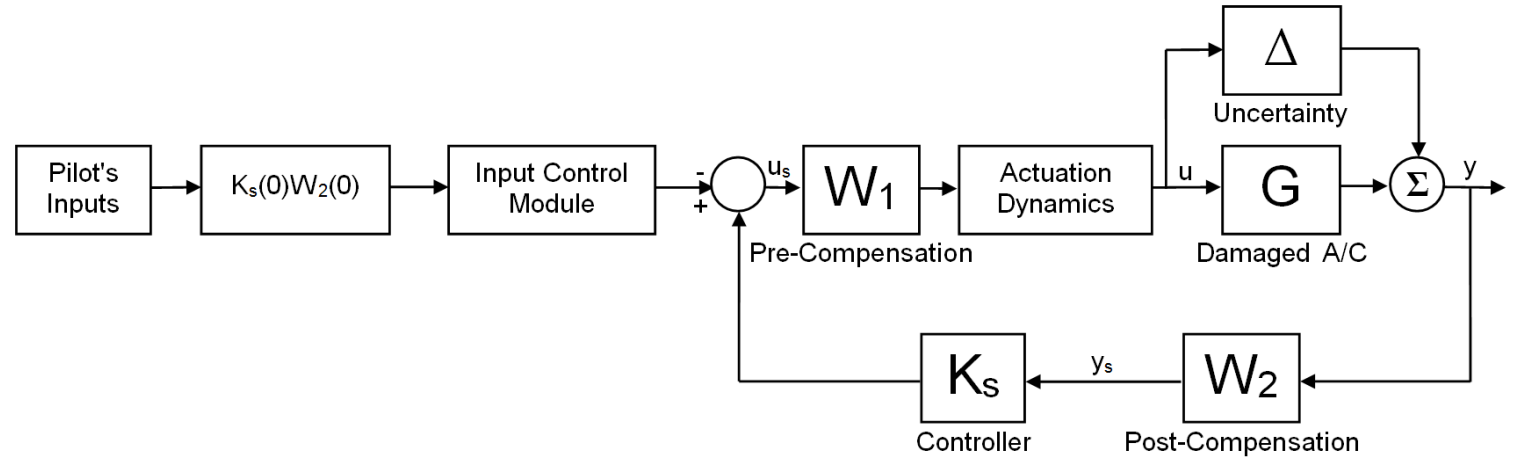

Figure 12.23: Block diagram for $H_{\infty}$ loop-shaping based robust control system design in the presence of uncertainty 
One thousand Monte-Carlo simulations were conducted to test the robustness of the damaged plant in the presence of uncertainty. The state responses in the presence of $30 \%$ of uncertainty are shown in Fig. 12.24 and Fig. 12.25, where it is obvious that the robust control system design is able to perform well under given uncertain conditions and the damaged aircraft has stable steady state responses within only 15 seconds. The roll angle $(\phi)$ settles at approximately between 3.1 and 3.3 degrees compared to about 3.2 degrees when there is no uncertainty. The roll rate $(p)$ settles at approximately between -0.02 and $0.01 \mathrm{deg} / \mathrm{s}$ compared to about 0 $\mathrm{deg} / \mathrm{s}$ when there is no uncertainty. The side-slip angle $(\beta)$ settles at approximately between -0.02 and -0.01 degree compared to about 0 degree when there is no uncertainty. Finally, the yaw rate $(r)$ settles at approximately between 0.14 and $0.16 \mathrm{deg} / \mathrm{s}$ compared to about $0.15 \mathrm{deg} / \mathrm{s}$ when there is no uncertainty. As for the heading angle $(\psi)$, the heading angle gain varies from 3.4 to 3.7 degrees compared to about 3.6 degrees when there is no uncertainty. In that sense, the uncertain plant dynamics are well within the expected bounds.

However, these favorable characteristics come at the expense of the control effort from the ailerons and differential thrust as shown in Fig. 12.26.

According to Fig. 12.26, when there is $30 \%$ full block, additive uncertainty, the aileron control demands the maximum deflection of approximately 2.4 degrees and reaches steady state between -0.12 and -0.09 degree after 15 seconds, which is quite similar to the required aileron control effort when there is no uncertainty. Therefore, the aileron control effort demands are reasonable and feasible due to the limiting factors of \pm 26 degrees of the aileron deflection [18] and the assumption that ailerons have instantaneous response characteristics by neglecting the lag from actuators or hydraulic systems.

As for differential thrust, when there is $30 \%$ uncertainty, the differential thrust 

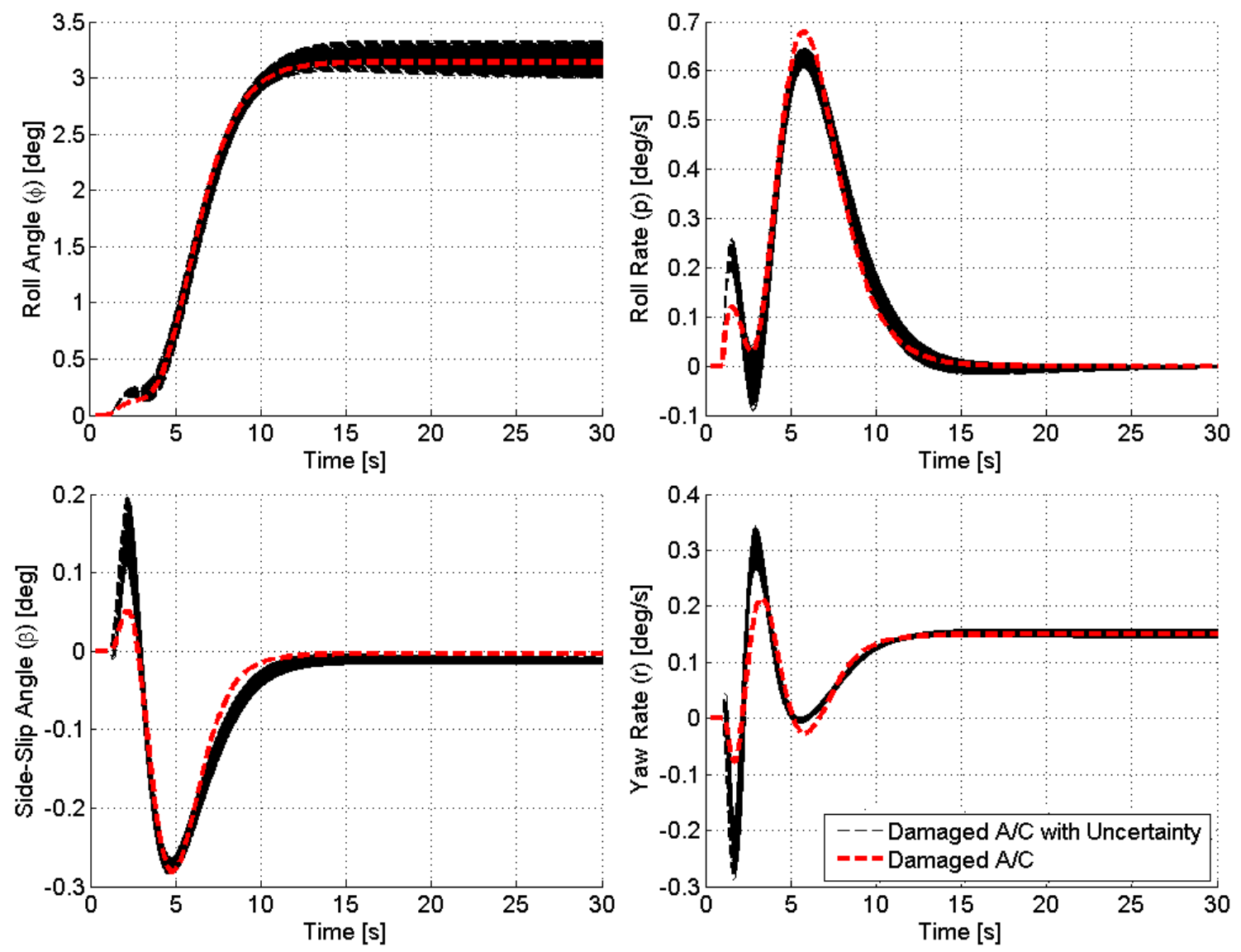

Figure 12.24: Closed loop responses of the damaged aircraft with a robust controller in the presence of $30 \%$ uncertainty 


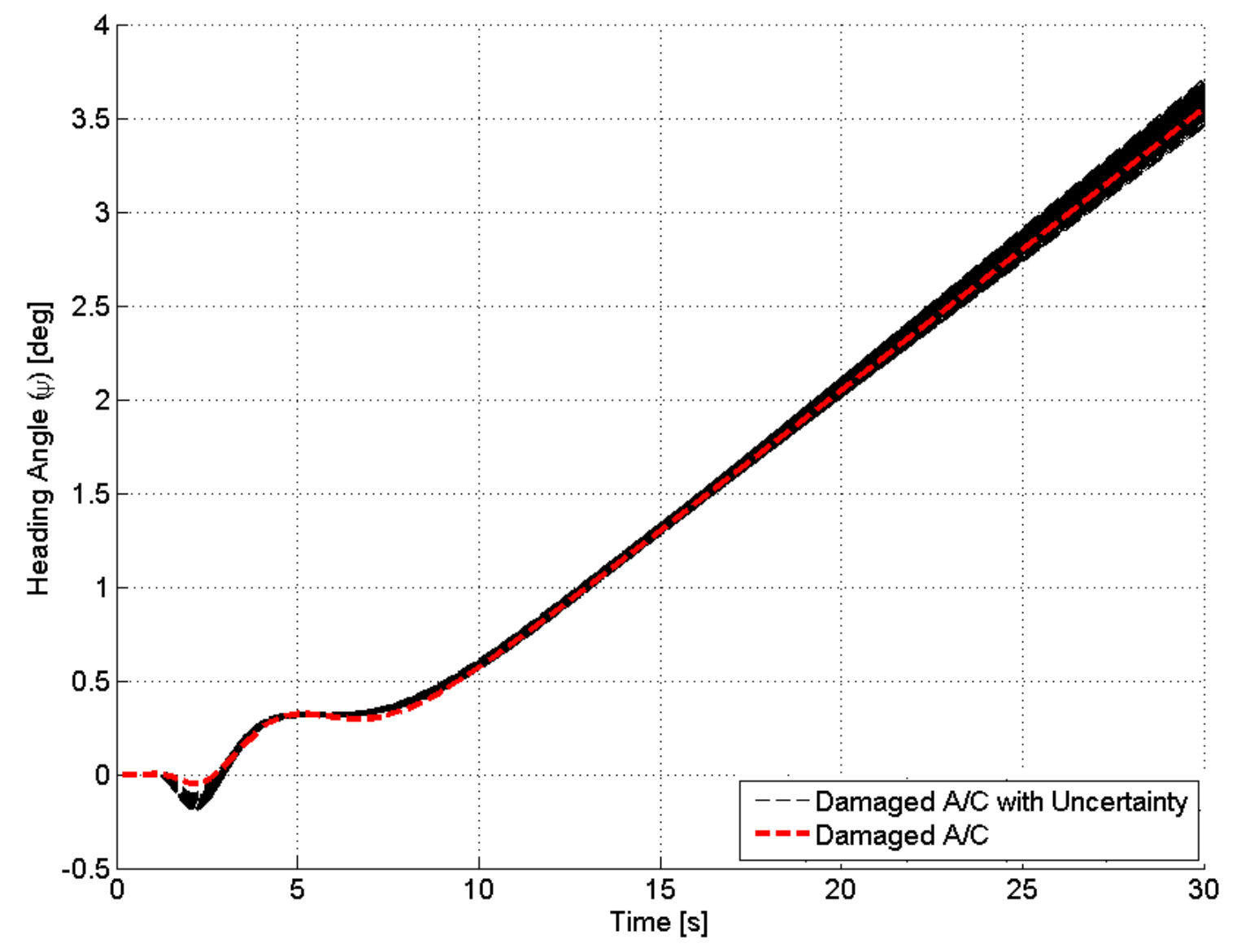

Figure 12.25: Closed loop heading angle response with a robust controller in the presence of $30 \%$ uncertainty 

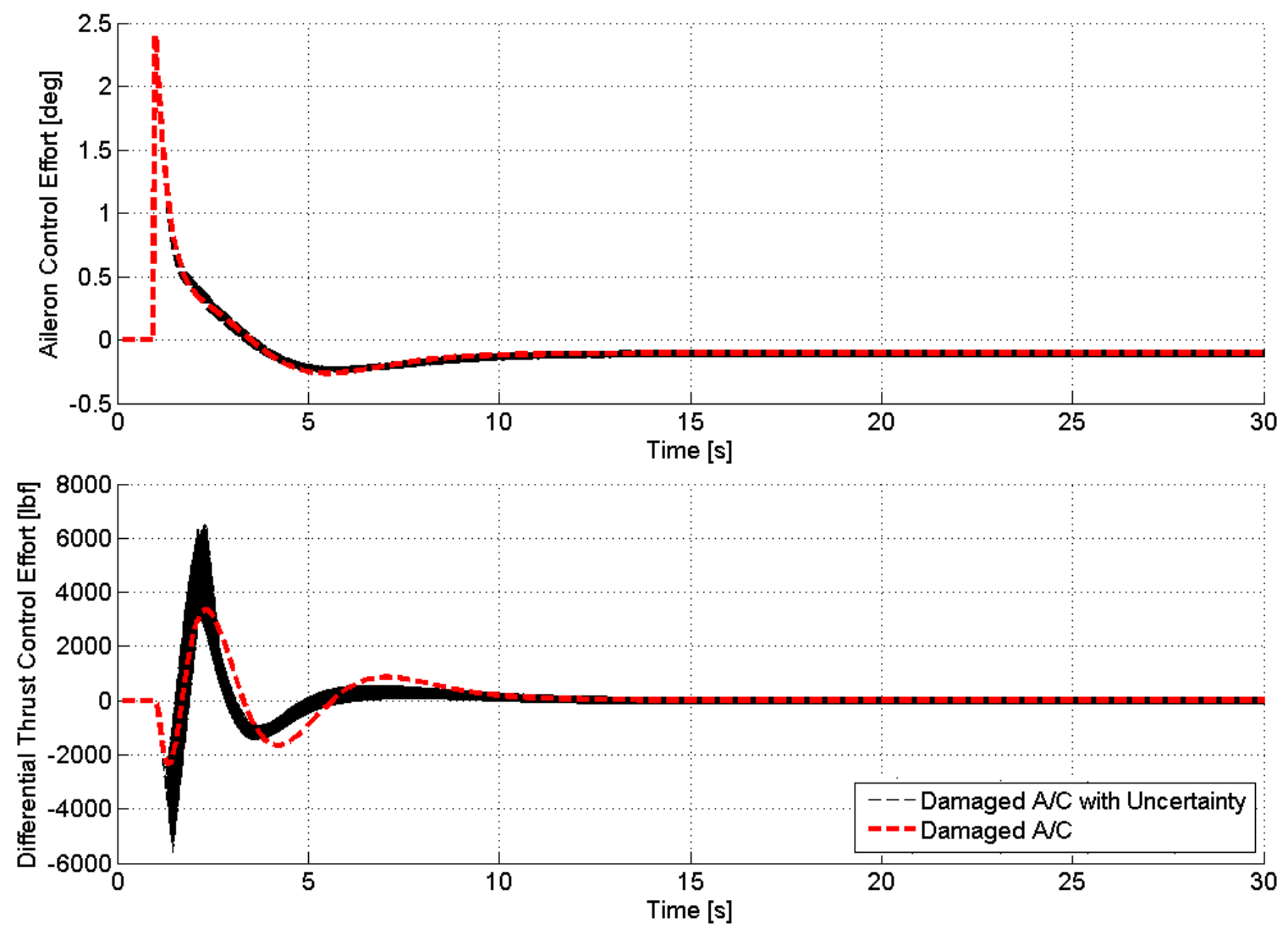

Figure 12.26: Closed loop control efforts with a robust controller in the presence of $30 \%$ uncertainty 
control demands at maximum approximately $6500 \mathrm{lbf}$, which is within the thrust capability of the JT9D-7A engine, and the differential thrust control effort reaches steady state between -120 lbf (negative differential thrust means $T 1<T 4$ ) and 120 lbf (positive differential thrust means T1 > T4) after 15 seconds. Compared to the case when there is no uncertainty, the demanded differential thrust associated with uncertain plant dynamics is higher in both magnitude and rate. It is also obvious from Fig. 12.26 that in a few cases, the differential thrust control effort demand hit the thrust generation rate limiter, which is set at $12,726 \mathrm{lbf} / \mathrm{s}$ for the JT9D-7A engine, but fortunately, the control system is so robust that throughout 1000 Monte-Carlo simulations, it can stabilize the aircraft's uncertain plant dynamics. Again, due to the differential thrust saturation value set at 43,729 lbf and the thrust rate limiter set at 12,726 lbf/s, this control effort of differential thrust in the presence of uncertainty is achievable in a real life situation.

\subsection{Chapter Summary}

In this chapter, we designed a $H_{\infty}$ loop-shaping based robust control system to stabilize the damaged aircraft. We also tested the robustness and sensitivity of the $H_{\infty}$ loop-shaping based robust control system design by introducing band-limited white noise as well as $30 \%$ full block, additive uncertainty into the damaged aircraft plant matrix. It has been concluded that the $H_{\infty}$ loop-shaping based robust control system was able to stabilize the damaged aircraft in only 15 seconds and under feasible control efforts without any actuator saturation. In the next chapter, we are conducting a comparison study of the LQR, adaptive, and $H_{\infty}$ loop-shaping based robust control System in order to investigate the merits and limits of each control methodology. 


\section{CHAPTER 13}

\section{COMPARISON OF LQR, ADAPTIVE, AND ROBUST CONTROL METHODOLOGIES}

\subsection{Comparison of LQR, Adaptive, and Robust Control Methodologies}

Throughout this thesis, three control systems (LQR, adaptive, and $H_{\infty}$ loop-shaping based robust control systems) have been designed to stabilize the damaged aircraft. In this chapter, a comparison study of the three control systems is conducted to investigate the merits and limits of each control system to save the damaged aircraft.

As seen in Fig. 13.1 and Fig. 13.2, all three control systems were able to stabilize the damaged aircraft within only 15 seconds. However, from Fig. 13.1, the $H_{\infty}$ loop-shaping based robust control system exceeds the other two control systems in its ability to eliminate entirely the side-slip angle and have a better overall steady-state yaw rate of $0.15 \mathrm{deg} / \mathrm{s}$ as compared to only $0.0057 \mathrm{deg} / \mathrm{s}$ achieved by the LQR and adaptive control systems. Furthermore, as seen in Fig. 13.2, the merit of the $H_{\infty}$ loop-shaping based robust control system is once again demonstrated by having an overall better heading angle gain of 3.6 degrees in 30 seconds compared to only 0.22 degree from the LQR and adaptive control systems. Next, the aileron and differential thrust control efforts for three types of control systems are illustrated in Fig. 13.3.

As discussed before in Chapter 10, Chapter 11, and Chapter 12, all three control systems demand the control efforts that are well within the capability of the 

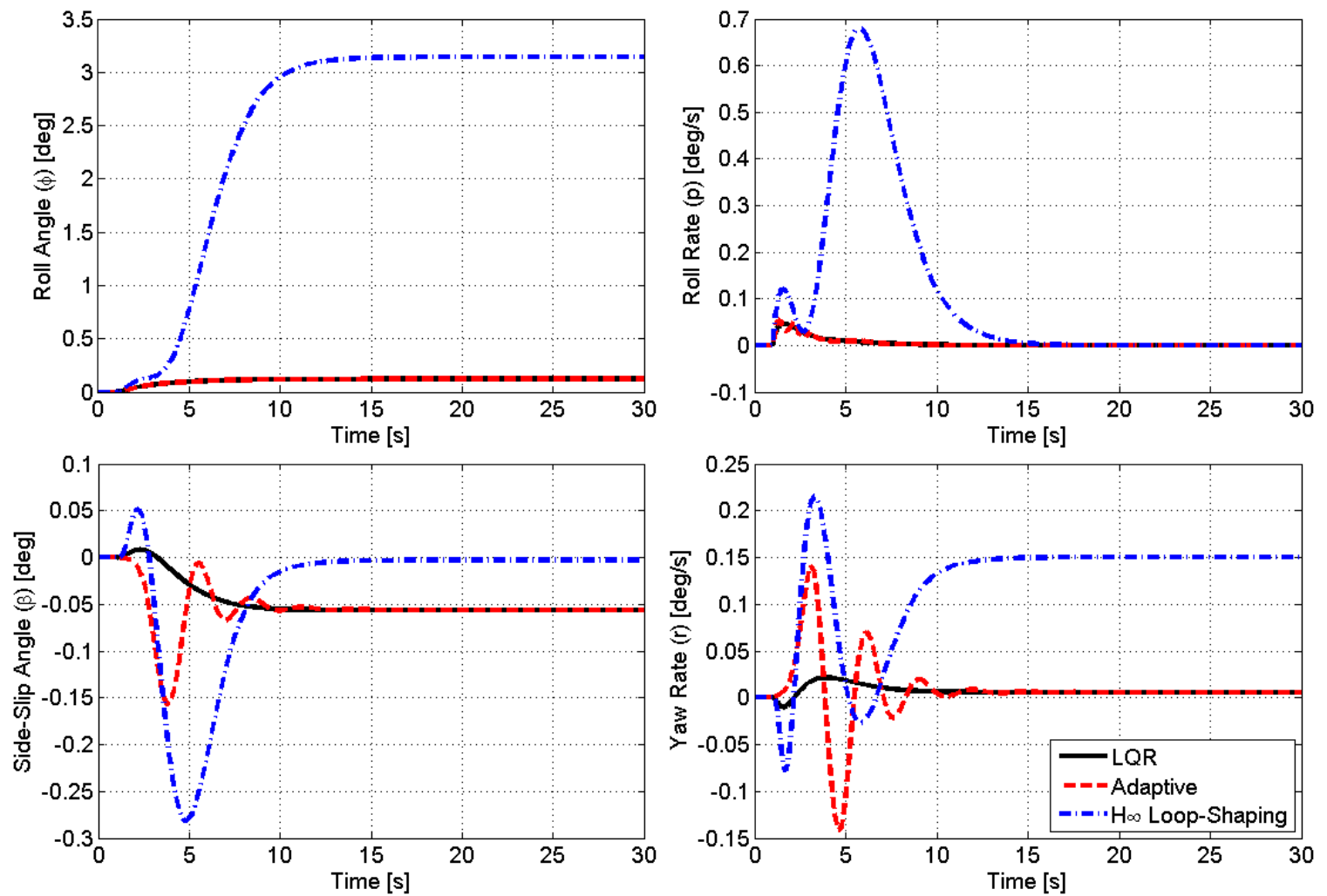

Figure 13.1: Comparison of closed loop responses of the damaged aircraft 


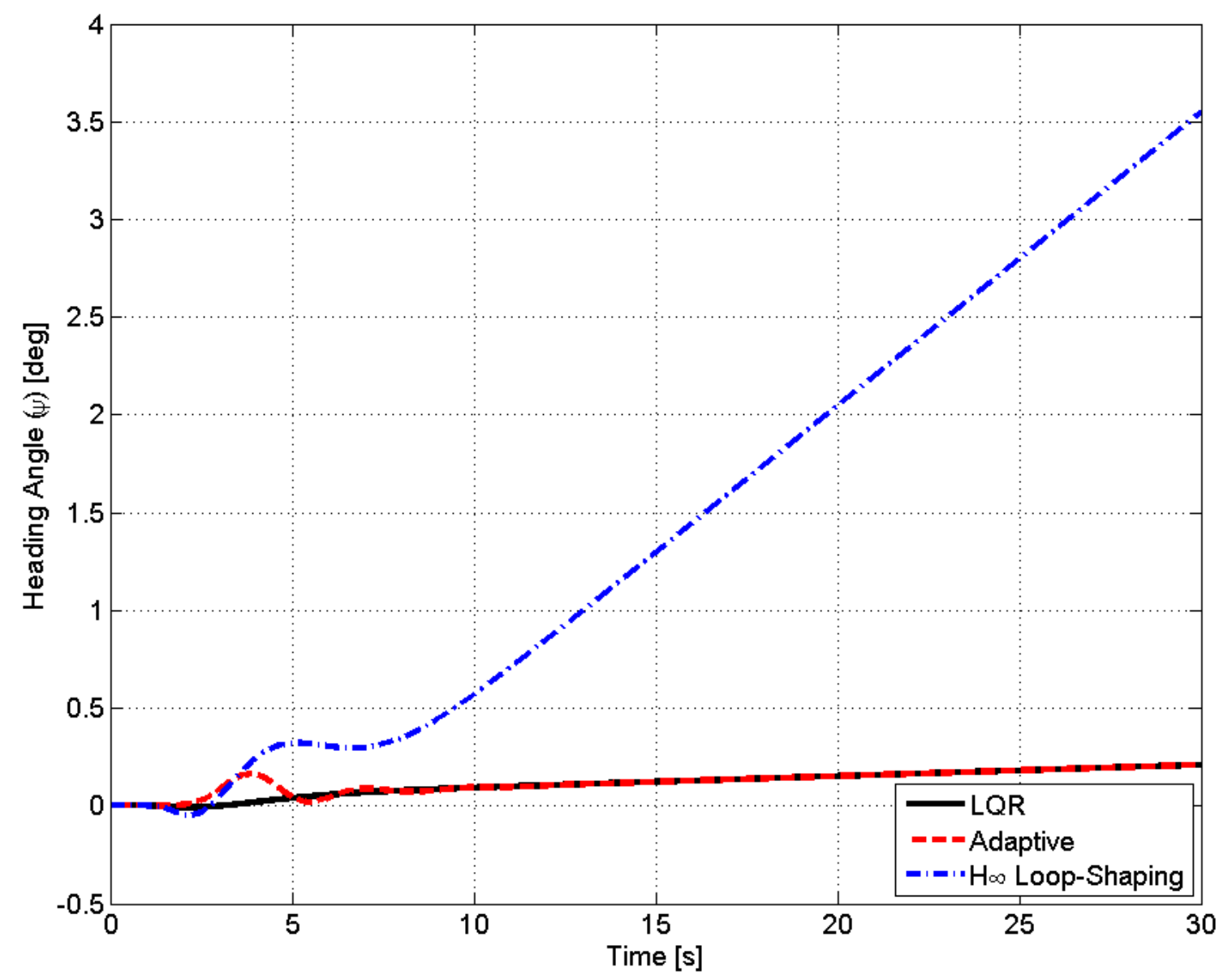

Figure 13.2: Comparison of closed loop heading angle responses 

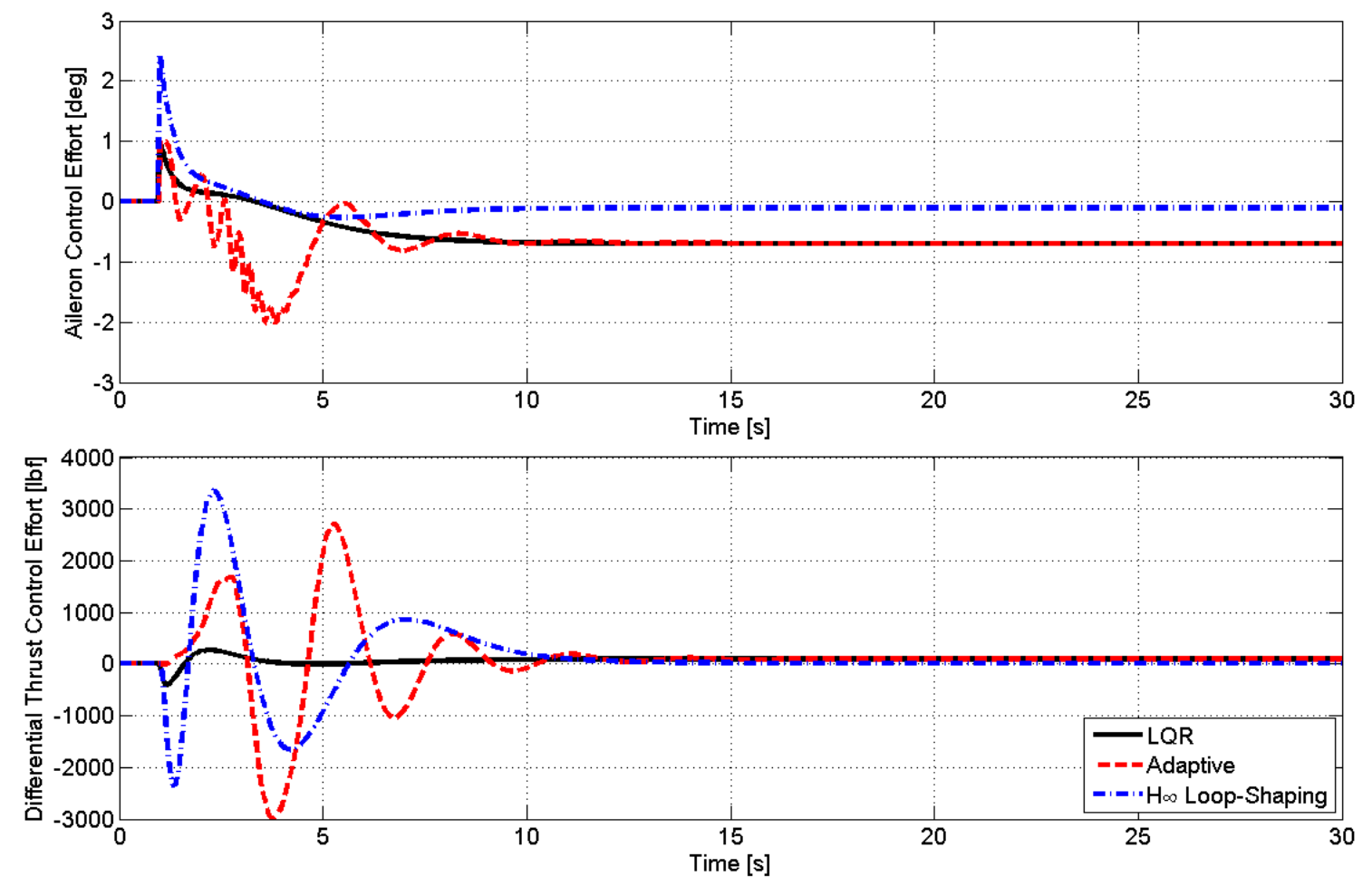

Figure 13.3: Comparison of control efforts 
ailerons and differential thrust. However, from Fig. 13.3, we can see that the LQR control system demands the least overall maximum control efforts from ailerons and differential thrust at 1 degree and -400 lbf as compared to only -2 degrees and -3000 lbf, and 2.4 degrees and $3350 \mathrm{lbf}$ by the adaptive and robust control system, respectively. However, for steady state control efforts, the $H_{\infty}$ loop-shaping based robust control system demands the least control efforts from ailerons and differential thrust at -0.1 degree and $15 \mathrm{lbf}$ as compared to only -0.7 degree and $85 \mathrm{lbf}$, and -0.7 degree and 1000 lbf by the adaptive and LQR control system, respectively. This is also another merit of the $H_{\infty}$ loop-shaping based robust control system because less demanded steady state aileron and differential thrust control efforts mean lower stress on the ailerons and engines.

Additionally, as discussed in Chapter 10.3, Chapter 11.3, and Chapter 12.3, from the robustness and uncertainty analyses in the presence of noise and uncertainty, the $H_{\infty}$ loop-shaping based robust control system excels above the other two types of control systems in terms of overall system robustness in the presence of band-limited white noise as well as uncertainty associated with the damaged aircraft plant dynamics.

It is also worth noting that the performance of the adaptive control system depends on its model plant (which is stabilized by an LQR controller), so the adaptive control system will follow and mimic the performance of the LQR control system. Therefore, it is possible to improve the performance of the model reference adaptive control system by using the model plant stabilized by the $H_{\infty}$ loop-shaping based robust control system. 


\subsection{Chapter Summary}

In this chapter, we conducted a comparison study of the LQR, adaptive, and $H_{\infty}$ loop-shaping based robust control system in order to investigate the merits and limits of each control methodology. We also concluded that due to its merits, the $H_{\infty}$ loop-shaping based robust control system provides the most remarkable results for stabilizing and saving the damaged aircraft under the assumptions in this thesis. 


\section{CHAPTER 14}

\section{CONCLUSIONS AND RECOMMENDATIONS}

\subsection{Conclusions}

This thesis studied the utilization of differential thrust as a control input to help a Boeing 747-100 aircraft with a damaged vertical stabilizer regain its lateral/directional stability. The motivation of this thesis is to improve the safety of air travel in the event of losing the vertical stabilizer by providing control means to safely control and save the damaged aircraft.

Throughout this thesis, the necessary nominal and damaged aircraft models were constructed, where lateral/directional equations of motion were revisited to incorporate differential thrust as a control input for the damaged aircraft. Then, the lateral/directional dynamic stability characteristics of the damaged aircraft were investigated. A frequency domain analysis was also conducted to study the response characteristics of the damaged aircraft. The engine dynamics of the jet aircraft were modeled as a system of differential equations with engine time constant and time delay terms to study the engine response time with respect to a commanded thrust input. Next, the novel differential thrust control module was presented to map the rudder input to differential thrust input. The controllability of the damaged aircraft was then investigated. The linear quadratic regulator controller was designed to stabilize the damaged aircraft. The ability of the damaged aircraft to track and mimic the behaviors of the model aircraft in an extreme scenario was illustrated through the adaptive control system design based on the Lyapunov stability approach. Next, the $H_{\infty}$ loop-shaping based robust control system design's ability 
to stabilize the damaged aircraft was proven as investigation results demonstrated that the damaged aircraft was able to reach steady state stability within only 15 seconds under feasible control efforts and without any actuator saturation. Results showed that the unstable open-loop damaged plant dynamics could be stabilized using all three control methodologies. Furthermore, the robustness and sensitivity analyses showed that all three control strategies were also able to stabilize the damaged aircraft plant in the presence of band-limited white noise as well as $30 \%$ full block, additive uncertainty. A comparison study of the LQR, adaptive, and robust control systems was also conducted to investigate the strengths and limits of each control system in order to select the most suitable control strategy to stabilize and save the damaged aircraft, and due to its merits, the $H_{\infty}$ loop-shaping based robust control system was found to have the most remarkable results for stabilizing and saving the damaged aircraft under the assumptions in this thesis.

Through listed analyses above, the ability to save the damaged aircraft by the three automatic control strategies and the utilization of differential thrust has been demonstrated in this thesis. This framework provides automatic control

methodologies to save the damaged aircraft and avoid the dangerous coupling of the aircraft and pilots, which led to crashes in a great number of commercial aircraft accidents.

\subsection{Recommendations}

For the proposed control methodologies to save the damaged aircraft to be feasible and effective in a real life scenario, the following recommendations should be taken into consideration.

- Aircraft Modeling: the damaged aircraft model in this thesis was 
constructed with a fully damaged vertical stabilizer. This represents an extreme scenario in which there is a total damage of the vertical stabilizer and the rudder. In a real life scenario, there will be no prior knowledge of how much the vertical stabilizer will be damaged so the parameters in the state matrix (A matrix) of the aircraft may vary. This was taken into account by the robustness and uncertainty analyses in this thesis.

- Propulsion Dynamics of Jet Engines: in this thesis, the non-linear and complex jet engine dynamics are simplified by the second-order linear model for analysis. It is worth noting that in a real life scenario, the differential thrust generation characteristics are governed by the highly non-linear and complex dynamics of jet engines. This can affect the effectiveness of differential thrust as a control input. Furthermore, due to the heavy dependence of the differential thrust response characteristics on the engine dynamics, in order to better incorporate the differential thrust as an effective control input in a life-saving scenario, major developments in engine response characteristics and engine dynamics modeling are desired to better assist such algorithms.

- Aileron Response Characteristics: it is worth taking into account that in this thesis, the ailerons are assumed to have instantaneous response characteristics by the assumption that there are no actuator and hydraulic lags associated with the ailerons. However, in a real life scenario, the actuator and hydraulic lags will affect the effectiveness of the ailerons. In addition, the rapid demand for aileron control effort can cause the ailerons to flutter, which may introduce vibration (and colored noise) into the damaged aircraft system. 
- Noise Sensitivity: the noise sensitivity analyses of control systems in this thesis are conducted by the introduction of the band-limited white noise to test the performance of the control systems in the presence of noise. It is worth noting that in a real life scenario, the damaged aircraft system can also be affected by colored noise.

- Other Disturbances: in this thesis, the aircraft is assumed to be in a steady, level cruise flight. However, in a real life scenario, there can be sudden, strong gusts of wind (such as updrafts or downdrafts) which can affect the performance of the control systems to save the damaged aircraft.

- Structural Integrity of the Damaged Aircraft: in a real life scenario, when the vertical stabilizer of an aircraft is ripped off, the structural integrity of the damaged aircraft is of great importance in deciding whether the damaged aircraft can be saved. 


\section{REFERENCES}

[1] National Transportation Safety Board, "In-Flight Separation of Vertical Stabilizer American Airlines Flight 587 Airbus Industrie A300-605R, N14053 Belle Harbor, New York, November 12, 2001," NTSB/AAR-04/04, 2004.

[2] Federal Aviation Administration, "Japan Airlines Flight 123, Boeing 747-SR100, JA8119: Accident Overview," U.S Department of Transportation, Federal Aviation Administration, Washington, DC.

[3] National Transportation Safety Board, "United Airlines Flight 232 McDonnell-Douglas DC-10-10, Sioux Gateway Airport, Sioux City, Iowa, July 19, 1989," NTSB/AAR90-06, 1990.

[4] Hartnett, F., "Ripped Off: The Story of No. 61-023," Barksdale Air Force Base, 2006.

[5] Bacon, B. J., and Gregory, I. M., "General Equations of Motion for a Damaged Asymmetric Aircraft," AIAA Guidance, Navigation, and Control Conference, AIAA Paper 2007-6306, 2007.

[6] Nguyen, N., and Stepanyan, V., "Flight-Propulsion Response Requirements for Directional Stability and Control," AIAA Infotech@Aerospace 2010, Atlanta, GA, 2010.

[7] Shah, G. H., "Aerodynamic Effects and Modeling of Damage to Transport Aircraft," AIAA Atmospheric Flight Mechanics Conference, Honolulu, HI, 2008.

[8] Burcham,F. W., Maine, T. A., and Burken,J. J., "Using Engine Thrust for Emergency Flight Control: MD-11 and B-747 Results," NASA/TM-1998-206552, May 1998.

[9] Guo, J., Tao, G., and Liu, Y., "Multivariable Adaptive Control of NASA Generic Transport Aircraft Model with Damage," Journal of Guidance, Control, and Dynamics, Vol.34, No.05, Sep.-Oct. 2011, pp. 1495-1506.

[10] Liu, Y., Tao, G., and Joshi, S. M., "Modeling and Model Reference Adaptive Control of Aircraft with Asymmetric Damages," 2009 AIAA Guidance, Navigation, and Control Conference, AIAA Paper 2009-5617, 2009. 
[11] Tao, G., and Ioannou, P. A., "Robust Model Reference Adaptive Control for Multivariable Plants," International Journal of Adaptive Control and Signal Processing, Vol. 2, No. 3, 1988, pp. 217248. doi:10.1002/acs.4480020305

[12] Urnes, J. M., Sr., and Nielsen, Z. A., "Use of Propulsion Commands to Control Directional Stability of a Damaged Transport Aircraft," AIAA

Infotech@Aerospace 2010, Atlanta, GA, 2010.

[13] Boeing, "747 Family: Technical Specifications- 747 Classics," The Boeing Company, 2014.

[14] Boeing, "747 Airplane Characteristics Airport Planning," The Boeing Company, 1984.

[15] Roskam, J., "Preliminary Calculation of Aerodynamic, Thrust and Power Characteristics," Airplane Design, Roskam Aviation and Engineering Corporation, Ottawa, KS., 1987.

[16] Nelson, R. C., Flight Stability and Automatic Control, $2^{\text {nd }}$ ed., WCB/McGraw-Hill, 1998.

[17] Ogata, K., Modern Control Engineering, $5^{\text {th }}$ ed., Prentice Hall, Upper Saddle River, NJ, 2010.

[18] Federal Aviation Administration, "Statistical Data for the Boeing-747-400 Aircraft in Commercial Operations," Federal Aviation Administration.

[19] Astrom, K. J., and Wittenmark, B., Adaptive Control, $2^{\text {nd }}$ ed., Addison-Wesley, Reading, MA, 1995.

[20] Vempaty, P. K., Cheok, K. C., and Loh, R. N. K., "Experimental Implementation of Lyapunov based MRAC for Small Biped Robot Mimicking Human Gait," Advances in Computer Science and Engineering, InTech, 2011.

[21] Ghaoui, L. E., "Optimization Models and Applications: Pseudo-Inverse of a Matrix," Department of Electrical Engineering and Computer Sciences, University of California, Berkeley.

[22] McFarlane, D., and Glover, K., "Robust Controller Design Using Normalized Coprime Factor Plant Descriptions," Vol. 138 of Lecture Notes in Control and Information Sciences, Springer-Verlag, Berlin, 1990.

[23] Skogestadt, S., and Postlethwaite I., Multivariable Feedback Control, John Wiley \& Sons, 1996. 